\title{
PERSONAL AND ORGANIZATIONAL PREDICTORS \\ OF SERVICE PROVISION FOR REFUGEES
}

A Dissertation
presented to
the Faculty of the Graduate School
at the University of Missouri-Columbia
In Partial Fulfillment
of the Requirements for the Degree
Doctor of Philosophy
EUNYOUNG JANG
Dr. Mansoo Yu, Dissertation Advisor
May 2018


The undersigned, appointed by the dean of the Graduate School of the University of Missouri-Columbia, have examined the dissertation entitled

\title{
PERSONAL AND ORGANIZATIONAL PREDICTORS OF SERVICE PROVISION FOR REFUGEES
}

presented by Eunyoung Jang, a candidate for the degree of doctor of philosophy, and hereby certify that, in their opinion, it is worthy of acceptance.

\author{
Professor Mansoo Yu
}

Professor Kelli Canada

Professor Sarah Myers Tlapek

Professor Duane Rudy 


\section{DEDICATION}

I want to dedicate this dissertation to my family members who have always supported, loved, and believe in me. I am very thankful for them and grateful they are my family. I would also like to dedicate my dissertation to the glory of God. 


\section{ACKNOWLEDGEMENTS}

I wish to acknowledge many individuals who helped me to achieve this doctoral degree. First and foremost, I want to give deep and sincere appreciation to my advisor Dr. Mansoo $\mathrm{Yu}$, who reviewed and revised my documents and offered counsel and encouragement through the entire doctoral journey. Without your patience, support, and guidance, this dissertation would not be possible. It was a great honor to work and study under your guidance. I also want to acknowledge the other members of my dissertation committee, Dr. Kelli Canada, Dr. Sarah Myers Tlapek, and Dr. Duane Rudy. My sincere gratitude goes to Dr. Canada who encouraged and believed in me. She gave me the opportunity to do research and provided invaluable guidance. I would like to thank Dr. Tlapek for her reviews and insights. Her insightful feedback made my dissertation strong. And, thanks go to Dr. Rudy for his time and attention to my work. In addition, I extend my thanks to all of the professors in the MU School of Social work who gave me their time, energy, and expertise. I also want to give my sincere gratitude to Dr. hyeji Choi who encouraged me to pursue the doctoral degree. She believed in me all the time.

I am extremely grateful to my friends and colleagues for their support and valuable prayers. Thank you for listening, offering me advice, and supporting me through this entire process. Church meetings, dinners, and general help and friendship were all greatly appreciated. They brought so much joy and happiness into my doctoral life.

I wish to thank the staff of Columbia Refugee and Immigrant Services and refugee service providers who were participants in my dissertation research. Without their support and contribution, my dissertation would not be complete. 
Finally, my thanks go to all the people who have supported me, directly or indirectly, through the long and challenging process of completing my doctoral degree. 


\section{TABLE OF CONTENTS}

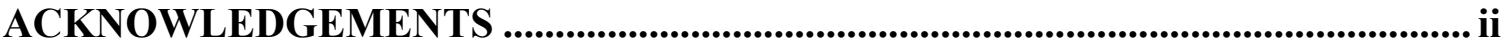

LIST OF TABLES ................................................................................................... viii

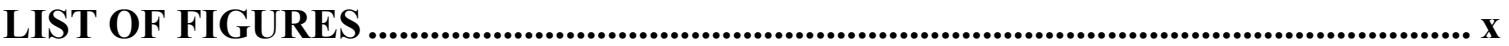

ABSTRACT ........................................................................................................................ xii

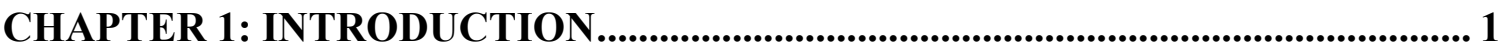

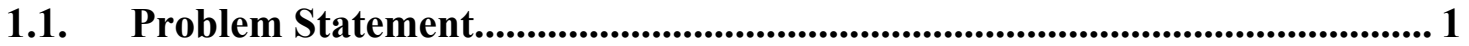

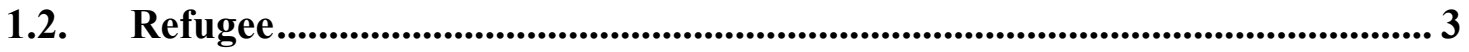

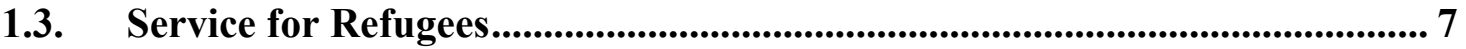

1.4. Service Provider for Refugees................................................................99

1.5. Purpose of the Research ................................................................................... 10

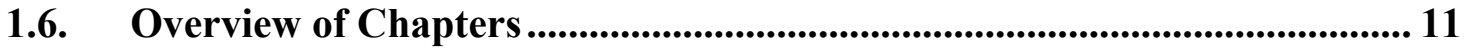

CHAPTER 2: THEORETICAL BACKGROUND AND ............................................ 12

LITERATURE REVIEW ............................................................................................... 12

2.1. The Gateway Provider Model (GPM) .................................................. 12

2.1.1. Behavioral Model.............................................................................. 13

2.1.2. Network Episode Model (NEM) N................................................. 14

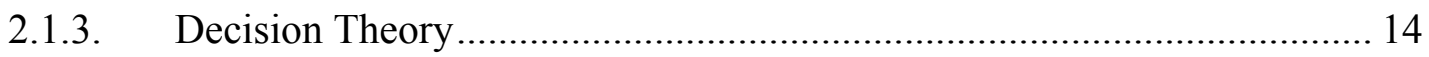

2.2. Cultural Competence Model (CCM) .......................................................... 16

2.3. Organization Theory: Organizational Culture .............................................. 20

2.4. Conceptual Model of the Study .......................................................... 24

2.5. Literature Review of Empirical Research ............................................... 25 
2.5.1. Personal Factors and Service Provision.................................................. 25

2.5.2. Organizational Culture Factors and Service Provision............................. 30

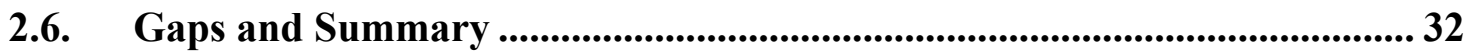

CHAPTER 3: RESEARCH DESIGN AND METHODS ................................................ 36

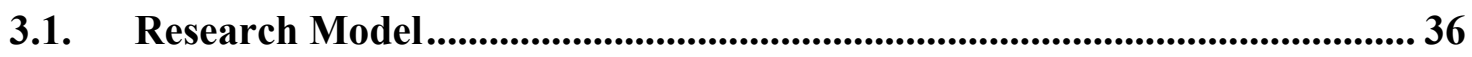

3.1.1 Research Model for Testing Direct Effects .................................................. 36

3.1.2 Research Model for Testing Indirect Effects ............................................. 37

3.2. Research Questions and Hypotheses ...........................................................38

3.3. Research Design ................................................................................................... 40

3.4. Participant Recruitment and Data Collection Procedure.............................. 40

3.5. Estimated Sample Size................................................................................... 45

3.6. Variables and Instrumentation............................................................................ 46

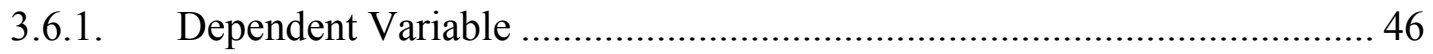

3.6.2. Service Providers' Personal Factors ………………………..................... 48

3.6.3. Organizational Culture Factors …………………................................. 52

3.6.4. Demographics and Other Information .................................................. 54

3.7. Data Analysis Strategies ...................................................................................... 57

3.8. Summary ............................................................................................................ 61

CHAPTER 4: RESULTS .................................................................................................. 62

4.1. Survey Procedure and Sample Size....................................................................... 62

4.2. Data Analysis Preparation ................................................................................... 63

4.3. Descriptive Statistics ............................................................................................... 65

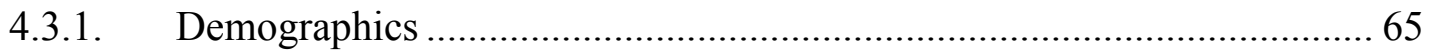




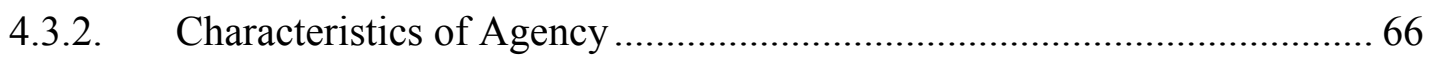

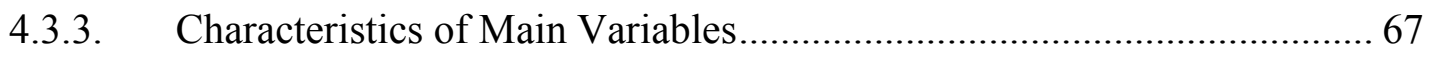

4.4. Bivariate Correlation Among the Study Variables.......................................... 82

4.5. Multiple Regression Analysis............................................................................... 86

4.5.1. Multiple Regression Analysis Predicting Overall Service Provision ....... 86

4.5.2. Multiple Regression Analysis Predicting Domains of Service Provision 91

4.5.3. Multiple Regression Analysis Predicting Domains of Service Referrals . 95

4.6. Mediation ................................................................................................................ 99

4.7. Summary ............................................................................................................ 120

CHAPTER 5: DISCUSSION AND IMPLICATIONS .................................................. 122

$5.1 \quad$ Discussion of Hypotheses..................................................................................... 123

$5.2 \quad$ Interpretation of the Major Findings............................................................... 131

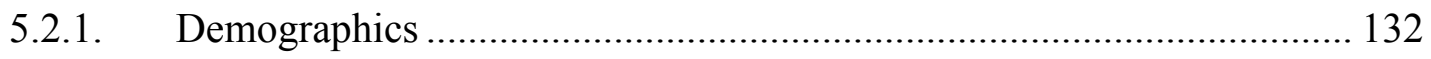

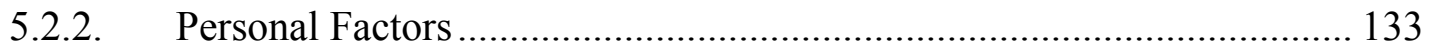

5.2.3. Organizational Culture Factors ……………………………………...... 137

5.3 Contribution to the Theories/Models ............................................................... 138

$5.4 \quad$ Implications …....................................................................................................... 140

5.4.1 Implication for Practice ............................................................................ 140

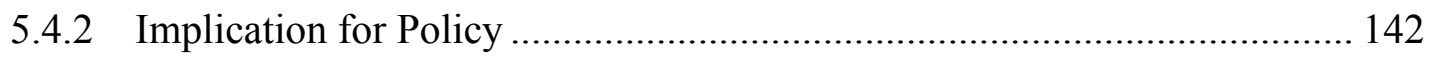

5.5 Strengths, Limitations and Recommendations for Future Research....... 145

5.5.1 Strengths of the Study ……………..................................................... 145

5.5.2 Limitation of the Study and Implication for Future Research .................... 145

5.6 Conclusion ............................................................................................................. 153 


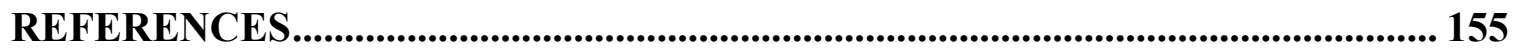

APPENDIX A: LIST OF ORGANIZATIONS ............................................................ 184

APPENDIX B: REFUGEE ARRIVALS BY STATE ................................................ 187

APPENDIX C: RECRUITMENT MAIL ..................................................................... 189

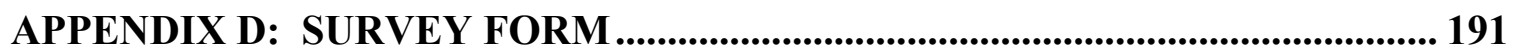

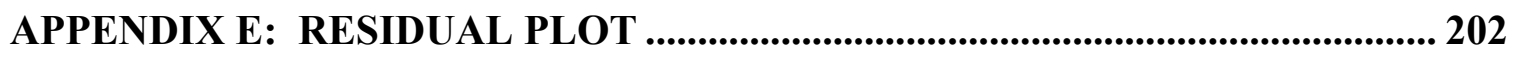

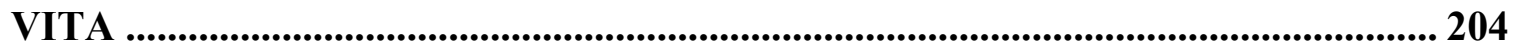




\section{LIST OF TABLES}

Table 1-1 Number of Refugees Admitted to the US from 2011 to 2013 .......................... 2

Table 3-1 Number of Agencies and Refugee Arrivals by Region ................................. 44

Table 3-2 Source of Knowledge of Refugee Instrument .......................................... 49

Table 3-3 Overview of Instruments ........................................................................ 56

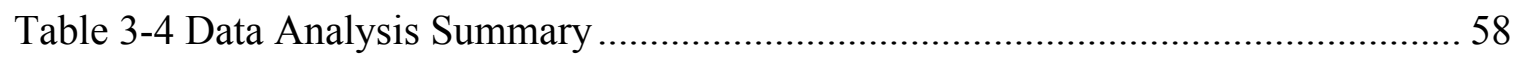

Table 4-1 Socio-demographic Characteristics of Study Participants $(\mathrm{N}=146) \ldots \ldots \ldots \ldots \ldots . . . . .65$

Table 4-2 Characteristics of Agency where Study Participants work $(\mathrm{N}=146) \ldots \ldots \ldots \ldots . . . .66$

Table 4-3 Range, Mean, and SD among Main Scale Variables $(\mathrm{N}=115) \ldots \ldots \ldots \ldots \ldots \ldots \ldots \ldots . . . . . . . . . . .67$

Table 4-4 Range, Mean, and SD among Dependent Variable by Domains $(\mathrm{N}=115) \ldots \ldots .69$

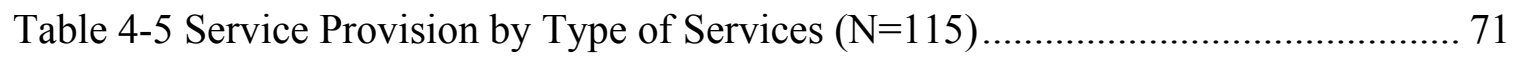

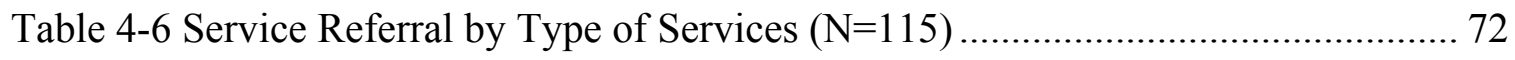

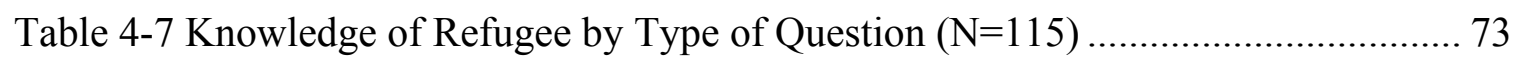

Table 4-8 Familiarity with Community Resources by Type of Question $(\mathrm{N}=115) \ldots \ldots \ldots .74$

Table 4-9 Cultural Competence Behavior by Type of Question $(\mathrm{N}=115)$..................... 75

Table 4-10 Cultural Awareness/Sensitivity by Type of Question $(\mathrm{N}=115)$................... 77

Table 4-11 Proficiency Organizational Culture by Type of Question $(\mathrm{N}=115) \ldots \ldots \ldots \ldots . . .78$

Table 4-12 Rigidity Organizational Culture by Type of Question $(\mathrm{N}=115)$.................. 79

Table 4-13 Resistance Organizational Culture by Type of Question $(\mathrm{N}=115)$............... 80

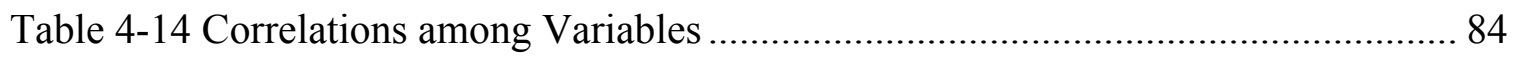

Table 4-15 Summary of Overall Multiple Regression Analysis for Variables Predicting

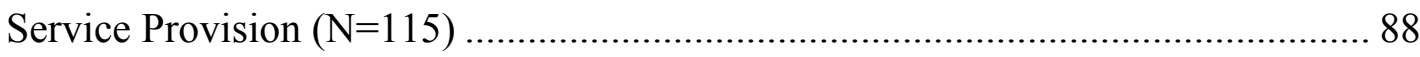


Table 4-16 Summary of Overall Multiple Regression Analysis for Variables Predicting Service Referral $(\mathrm{N}=115)$.

Table 4-17 Summary of Multiple Regression Analysis for Variables Predicting Service

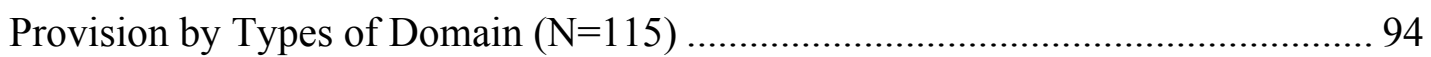

Table 4-18 Summary of Regression Analysis for Variables Predicting Service Referral by

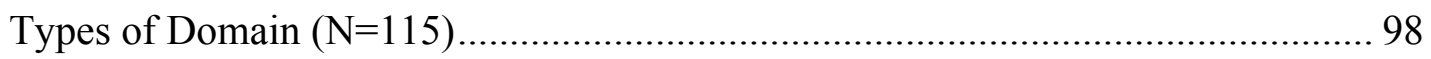

Table 4-19 Regression Coefficients, Standards Errors, and Model Summary Information for the Parallel Multiple Mediator Model Depicted in Figure 4-1....................... 102 Table 4-20 Regression Coefficients, Standards Errors, and Model Summary Information for the Parallel Multiple Mediator Model Depicted in Figure 4-2 ....................... 108

Table 4-21 Regression Coefficients, Standards Errors, and Model Summary Information for the Parallel Multiple Mediator Model Depicted in Figure 4-3........................ 113

Table 4-22 Regression Coefficients, Standards Errors, and Model Summary Information for the Parallel Multiple Mediator Model Depicted in Figure 4-4....................... 118

Table 5-1 Service Ranking of Most Frequently Provided and Referred ....................... 125 


\section{LIST OF FIGURES}

Figure 1-1 Simplified infographic of the refugee screening process (White House, 2015) 6

Figure 2-1 Gateway Provider Service Framework (Stiffman et al., 2004)....................... 13

Figure 2-2 Contextual Model of Cultural Competence ……………………………....... 20

Figure 3-1 Research model for testing direct associations of multiple variables in

predicting service provision

Figure 3-2 Research model for testing direct associations of multiple variables in predicting referral

Figure 3-3 Research model for testing indirect associations of multiple variables in

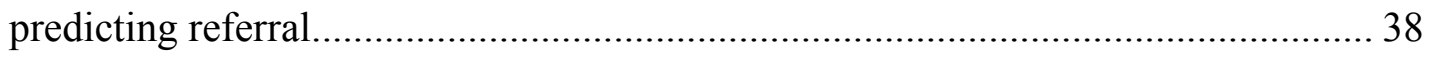

Figure 3-4 Data Collection Procedure ........................................................................... 42

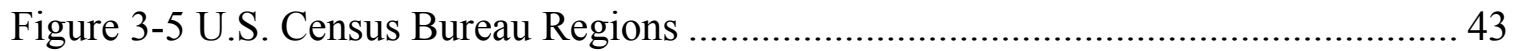

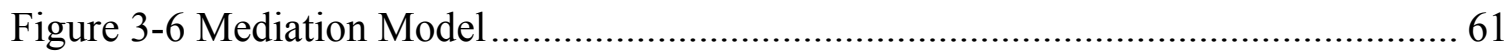

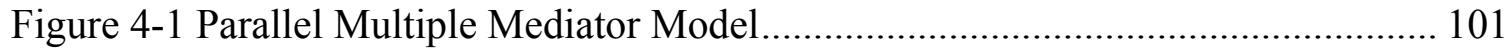

Figure 4-2 Cultural Competence Mediate Association between Familiarity with

Community Resources and Service Provision by Six Domains .............................. 104

Figure 4-3 Parallel Multiple Mediator Model 2.......................................................... 107

Figure 4-4 Cultural Competence Mediate Association between Familiarity with Community Resources and Service Referrals by Domains .................................... 110

Figure 4-5 Parallel Multiple Mediator Model 3 …………............................................. 112

Figure 4-6 Cultural Competence Mediate Association between Work Experience and

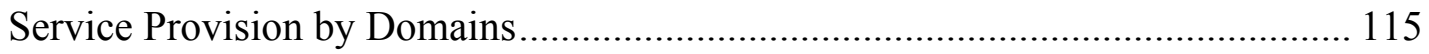

Figure 4-7 Parallel Multiple Mediator Model 4............................................................ 117 
Figure 4-8 Cultural Competence Mediate Association between Work Experience and Service Referrals by Domains 120 


\title{
PERSONAL AND ORGANIZATIONAL \\ PREDICTORS OF SERVICE PROVISION FOR REFUGEES
}

Eunyoung Jang

Dr. Mansoo Yu, Dissertation Supervisor

\begin{abstract}
This cross-sectional research study examined related predictors such as knowledge of refugees, familiarity with community resources, cultural competence, and organizational culture factors associated with service provision for refugees among a sample of service providers. Three theories/models provided the theoretical frameworkthe gateway provider model (Stiffman, Pescosolido, \& Cabassa, 2004), cultural competence model (Schim, Doorenbos, \& Borse, 2005; Suarez-Balcazar et al., 2011), and organizational theory (Glisson et al., 2008a; Glisson et al., 2008b). This study aimed to 1) describe the characteristics of a sample of refugee service providers, and 2) examine how service providers' personal factors and organizational culture factors are associated with service provision.

One hundred seventy refugee service providers across the United States completed an online survey. Descriptive statistics, bivariate analysis, multiple regression analysis, and mediation analysis were employed to address four research questions and related hypotheses. Seventy-five point three percent of study participants were white and $81.5 \%$ were female. The age of subjects ranged from 23 to 75 years of age. Most $(60 \%)$
\end{abstract}


of the study participants completed a graduate degree or higher and $86.3 \%$ of participants had not been a refugee before. Further, $62.3 \%$ of study participants were employed in a refugee resettlement related job. Most of the respondents worked in the Midwest $(35.6 \%)$ and South $(36.3 \%)$ regions. The vast majority of participants $(84.9 \%)$ were working at a refugee resettlement agency. The major findings indicated that service providers' personal factors (i.e., knowledge of refugees, familiarity with community resources, and cultural competence) were significantly associated with overall service provided and referrals as well as each domain of service provided and referrals. Proficient organizational culture was positively associated with service provided and referrals, and constantly rigid organizational culture was negatively associated with service provided and referrals. Cultural competence behavior had a mediation effect on the association between service providers' familiarity with community resources and service provision. In addition, cultural competence behavior had a mediation effect on the association between service providers' work experiences and service provision.

The findings provide important insights for refugee service providers, refugee service organizations, researchers, and policy makers for improving refugee service by developing a cultural competence training program and an online networking system. 


\section{CHAPTER 1: INTRODUCTION}

\subsection{Problem Statement}

More than 17 million refugees are receiving protection and assistance from the office of the United Nations High Commissioner for Refugees (UNHCR) due to the growing number of crises worldwide (UNHCR, 2017). These problems are not expected to improve in the near future. UNHCR helps refugees resettle in another country but, unfortunately, very few refugees have the opportunity to be resettled (UNHCR, n.d.). A small number of countries are currently accepting refugees, including the United States (US), Canada, Australia, and some European nations (e.g., Sweden, Germany, and United Kingdom). As shown in Table 1-1, 69,926 refugees were admitted into the United States in 2013, the number gradually increased to 84,995 in 2016 (Refugee Processing Center, 2018). However, after Executive Order 13780 titled 'Protecting the Nation From Foreign Terrorist Entry Into the United States', signed on January 27, 2017 by President Donald Trump suspended refugee admission, the planned number of refugees allowed into the U.S. in fiscal 2017 declined from 110,000 to 50,000 (Pew Research Center, 2017). Moreover, the declining trend in refugee admission was continuous. According to the report of proposed refugee admissions in FY 2018, the ceiling is 45,000 (Bureau of Population, Refugees, and Migration, 2017). However, since 1980, when Congress passed the Refugee Act, about 13 million refugees have been resettled in the U.S., with a lot of them still residing in the U.S. 
Table 1-1 Number of Refugees Admitted to the US from FY2011 to FY2018

\begin{tabular}{|l|c|c|c|c|c|c|c|c|}
\hline & 2011 & 2012 & 2013 & 2014 & 2015 & 2016 & 2017 & $2018^{1}$ \\
\hline Total & 56,384 & 58,238 & 69,926 & 69,987 & 69,933 & 84,995 & 53,716 & 10,548 \\
\hline
\end{tabular}

Living in a new environment can be an exciting and stimulating experience (Smith, n.d.), but living in a culture different from your own may create challenges and hardships (Kovacev \& Shute, 2004). Many refugees come to the US after struggling for survival or experiencing other difficult situations. They are then faced with new challenges, such as learning English and becoming accustomed to local values and norms (Sabbah, 2007). Refugees retain much of their native culture while adapting to their host country (Henry, 2012). Thus, they may experience inner conflicts between their native culture and their host culture (Henry, 2012), which may lead to physical and psychosocial distress (Marotta, 2003; Silove et al., 2006). Mollica (1990) described those experiences as a "social earthquake." They are often also attempting to recover from traumatic past experiences, which adds to the potential acculturation stress of adjusting to a new environment. They, however, don't have any roots to support them at a time when an emotional and social support system is essential.

Therefore, in the US, many refugee service centers or agencies provide basic services for refugees (Kenny \& Lockwood-Kenny, 2011). Service providers are central to the settlement process and are unique as they are the point of transmission between the government system and clients (Mukhtar, 2013). The role of service providers, including 
caseworkers, health providers, interpreters, and educators, as the first people who meet refugees, is an important one. Refugees trust and rely on their service providers to obtain information and help them access services. Therefore, the role of service providers is crucial as they are actively engaged in responding to newly arrived refugees and thinking about how and why it is important to include them in their communities.

Nevertheless, there is little available research about refugee service providers. As a result, important factors associated with service provision for refugees are largely unknown. A service provider may be quite capable, but they might follow their organizational culture. Therefore, the personal and organizational factors that a service provider faces should be considered when studying their service provision. Combining the gateway provider model, cultural competence model, and organization theory as a theoretical framework, this study examined how social service provision for refugees is effected by the service provider's knowledge of refugees, familiarity with community resources, cultural competence, and organizational culture factors.

\subsection{Refugee}

Refugees share similar characteristics with immigrant groups that experience intercultural change since they are confronted with the demands of adjusting to an unfamiliar environment (Ward, Bochner, \& Furnham, 2001). However, refugees are considered a special segment of the population and are commonly distinguished from immigrants; a refugee is forced to migrate while immigrants move voluntarily (Black, 2001). Involuntary migrants have greater difficulty accepting their new culture than voluntary migrants who choose to migrate for better economic or educational 
opportunities (Ogbu \& Simons, 1998).

Who, exactly, is a refugee? The United Nations' (UN) 1951 Geneva Convention defines a refugee as someone who "owing to a well-founded fear of being persecuted for reasons of race, religion, nationality, membership of a particular social group or political opinion, is outside the country of his nationality, and is unable to, or owing to such fear, is unwilling to avail himself of the protection of that country" (United Nations High Commissioner for Refugee, 2011). Additionally, in the United States, section 101(42)(a) of the Immigration and Nationality Act (INA) legally defines a refugee as "any person who is outside any country of such person's nationality or, in the case of a person having no nationality, is outside any country in which such person last habitually resided, and who is unable or unwilling to return to, and is unable or unwilling to avail himself or herself of the protection of, that country because of persecution or a well-founded fear of persecution on account of race, religion, nationality, membership in a particular social group, or political opinion..." (U.S. Citizenship and Immigration Services, n.d.). As indicated in the above definitions, refugees experience a multitude of threatening circumstances resulting in their migration to other countries (Oliver, 2014).

Refugees who wish to come to the United States must meet the UNHCR definition to be eligible for refugee status (Martin \& Yankay, 2013) and they must be cleared by the legal immigration process in the US. The United States Refugee Admittance Program (USRAP) allows refugees to arrive in the US in one of three ways: 1) via referral from the UNHCR, 2) through family members who have already resettled in the US, or 3) by being a member of a designated group of special humanitarian concern (Kenny \& Lockwood-Kenny, 2011; USCIS, 2016). All applicants for refugee 
status are physically located outside of the US (Martin \& Yankay, 2013). After the application has been submitted, the Overseas Processing Entity (OPE) screens the applicants and refers them to the Bureau of U.S. Citizenship and Immigration Services (USCIS) for an interview; less than $1 \%$ of the global refugee population is accepted during this process (The Whitehouse, 2015). Once the refugee passes the background, biodata, and medical screening processes, they are assigned to a sponsoring organization that helps them resettle in the US. Applicants can be rejected or held if any of these reviews detect any issues, serious health matters, moral and criminal matters, or security issues. Finally, the International Organization for Migration (IOM) arranges a flight. Although these steps appear to be simplistic, the average processing time is 12 to 18 months (U.S. Department of State, 2013) (See Figure 1-1). 


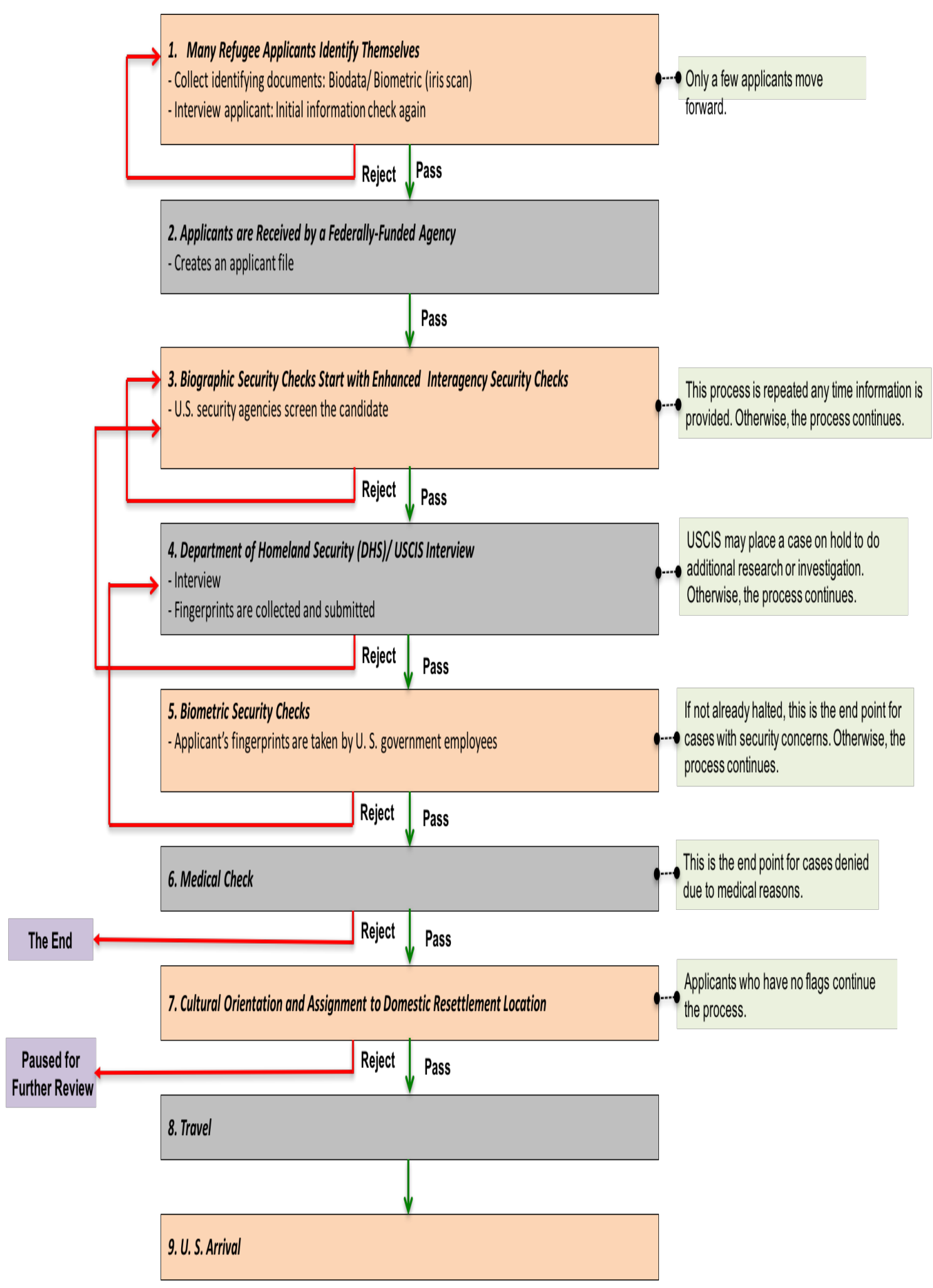

Figure 1-1 Simplified infographic of the refugee screening process (White House, 2015) 


\subsection{Service for Refugees}

The US resettlement programs provide service through public and private nonprofit organizations to help refugees become self-sufficient as quickly as possible (UNHCR, 2014). The federal government and voluntary organizations began to coordinate refugee resettlement assistance in the 1930s (Brown \& Scribner, 2014). Under President Roosevelt's directive, humanitarian agencies were allowed to become the sponsoring agencies of refugees. The federal government supported the cost of travel from Europe to the port of entry and agencies provided for the needs of refugees after their arrival. At the time, there was no legal distinction between refugee and immigrant (Brown \& Scribner, 2014).

Following World War II and during the Cold War era, large groups of people living in communist countries in Europe were displaced from their homes and forced to flee (Drachman, 1995). Several major refugee crises occurred following the World War II crisis: the Hungarians in 1956, Cubans in 1960, and Indochinese in 1975. Humanitarian agencies were important organizations during those crises; however, refugee support programs have been improved and, finally, a permanent and consistent refugee policy enacted — the Refugee Act of 1980. Today's US Refugee Admission Program and services are based on the Refugee Act (Refugee Council USA, n.d.).

Refugees have many needs, and sponsoring agencies should provide services that follow the UNHCR resettlement guideline. Other public and private social service agencies in diverse settings, such as hospitals and mental health organizations, schools, and workplaces, which assist all people in need, also provide essential services for refugees (Drachman \& Ryan, 2001). Common services could be categorized into six 
domains: basic needs, orientation, assistance to access benefits and services, employment, health care, and other services (UNHCR, 2014). Basic needs include airport reception, initial housing, essential furnishings and supplies, and clothing. Food is also provided based on the newcomers' nationality and religion. Moreover, financial assistance is provided based on the number of family members and income. Several different types of orientations exist, but it is mandatory for caseworkers to provide cultural orientation for refugees on their first or second day in the US. Cultural orientation includes basic information on US life, such as how to use a kitchen, personal and public safety, and the standard of personal hygiene. In addition, baseline rules and regulations are provided, such as smoking and drinking rules. Refugees are also provided with information on applying for a social security number, the Supplemental Nutrition Assistance Program (SNAP; formally called food stamps), school enrollment for school-aged kids, and English class enrollment for adults. Moreover, agencies help with finding job opportunities based on the refugee's capabilities, job orientation, job interview training, and follow-up assessment. Medicaid or refugee medical assistance is provided by ORR to cover basic health care costs for up to eight months. Refugees are provided a physical, as well as mental health treatment, through those programs. Other services and supports vary by state and agency, depending on their funding situation, and could include transportation service for job interviews and doctor's visits, transportation training, legal service, advocacy, or counseling service. Further, refugees are a group within our society that needs help; therefore, they should be provided social services. Social services are those programs designed to enhance individual, family, or group functioning. In this 
broad sense, social service refers to universal services used by everyone (Kahn, 1979). Thus, all social services include service to refugees.

In this study, service provision is defined as the number of times service is provided for refugees. Due to the characteristics of the organization, service providers might not be able to provide direct service to refugees (e.g., in the health care center they do not provide housing service to refugees). However, they could serve as the primary organization to resolve the situation and give information to the refugees (Hughes, Barker, Kemenoff, \& Hart, 1993); we call this "referral” (e.g., a health care center could refer a refugee to an appropriate organization that can help the refugee find a house). According to Wong-Tam (2003), referral is defined as researching information or referring clients to relevant services and/or resources within the agency or broader community according to the client's identified need(s). A referral is one of the most common ways to link clients to services (Bunger, 2010; Provan, 1984). Therefore, direct service provision and referrals were used in this study to capture service provision for refugees.

\subsection{Service Provider for Refugees}

There is no standard definition for refugee service providers. This may be because there are several different types of providers who assist refugees. Refugee service providers are members of a particular community whose task is to assist with refugee resettlement in cooperation with social service agencies or the health care system. Even when they cannot offer direct services, their referrals, consultations, and communications with potential service providers help refugees access services, and they serve as a link 
between the system and the refugee (Schwartz, Unger, Zamboanga, \& Szapocznik, 2010). Service providers ideally possess certain skills and capacities that are important to gaining the trust and acceptance of refugees. These may include cultural communication and mediation skills, understanding cultural health belief systems and their health issues, knowledge of refugee situations, the ability to advocate for and protect refugees, and cultural sensitivity. In this study, a service provider is defined as any person who works within refugee support services. Moreover, a broader conceptualization of services would include varying degrees of expertise, such as social workers, case managers, , ESL teachers, school counselors, therapists, and employment counselors, who have provided needed services and referrals (Wong-Tam, 2003).

\subsection{Purpose of the Research}

Using a combined theoretical framework based on three models/theories - the gateway provider model (Stiffman, Pescosolido, \& Cabassa, 2004), cultural competence model (Schim, Doorenbos, \& Borse, 2005; Suarez-Balcazar et al., 2011), and organizational theory (Glisson et al., 2008a; Glisson et al., 2008b)—-this study examined related predictors such as knowledge of refugees, familiarity with community resources, cultural competence, and organizational culture factors associated with service provision for refugees among a sample of service providers.

This study has the following aims:

- describe the characteristics of a sample of refugee service providers; and

- examine how service providers' personal factors and organizational culture factors are associated with service provision. 
Insight into these topics has the potential to better understand providers of services for refugees. Results of the study also lead to recommendations to optimize service provision and utilization.

\subsection{Overview of Chapters}

This study consists of five chapters. Chapter 1 outlines the introductory information and important concepts for this study. Chapter 2 explains the models/theories used to conceptualize service providers' service provision and discusses the literature relevant to the proposed study. Based on the theoretical framework and literature reviews, Chapter 3 presents research models and hypotheses for this study and the methodology, including sampling, instruments, data collection procedure, and analysis of data. Chapter 4 displays the findings of the research. Finally, Chapter 5 provides an interpretation of the study findings, implications, study limitations, and recommendations for future studies.

The next chapter begins by outlining the theoretical framework and continues with a literature review of empirical research. 


\section{CHAPTER 2: THEORETICAL BACKGROUND AND LITERATURE REVIEW}

As noted in the previous chapter, this study aims to investigate the factors related to service provision for refugees by service providers. In order to address this aim, the study applied three models/theories to create a theoretical framework. The models/theories used in this framework are not specific to research on refugee service providers because few studies exist to guide this field; however, it is important to understand what factors may related to the service provision that providers give to refugees. Since one single model/theory cannot serve to examine every aspect of personal and organizational dimensions, this study uses multiple models/theories to identify possible factors. This study took a first step toward holistically understanding service providers' service provision and building knowledge of refugee service providers' perspectives. Moreover, these models/theories guided the development and selection of the proposed variables and research questions. The three models/theories that have been merged to construct a theoretical framework for this study are the gateway provider model, the cultural competence model, and organizational theory.

\subsection{The Gateway Provider Model (GPM)}

The GPM was originally developed by Stiffman, Pescosolido, and Cabassa (2004) to help children and adolescents with mental problems access mental health services. They focused their research on factors that affect youth access to mental health services or treatments (see Figure 2-1). In this study, the GPM draws general concepts such as the idea that there is a key person (or gateway provider) who affects the response to service provision for those clients with mental health problems (Stiffman, Pescosolido, \& 
Cabassa, 2004). This model is an elaboration of three existing complementary

models/theories: the behavioral model, the Network Episode Model (NEM), and decision theory (Stiffman, Pescosolido, \& Cabassa, 2004).

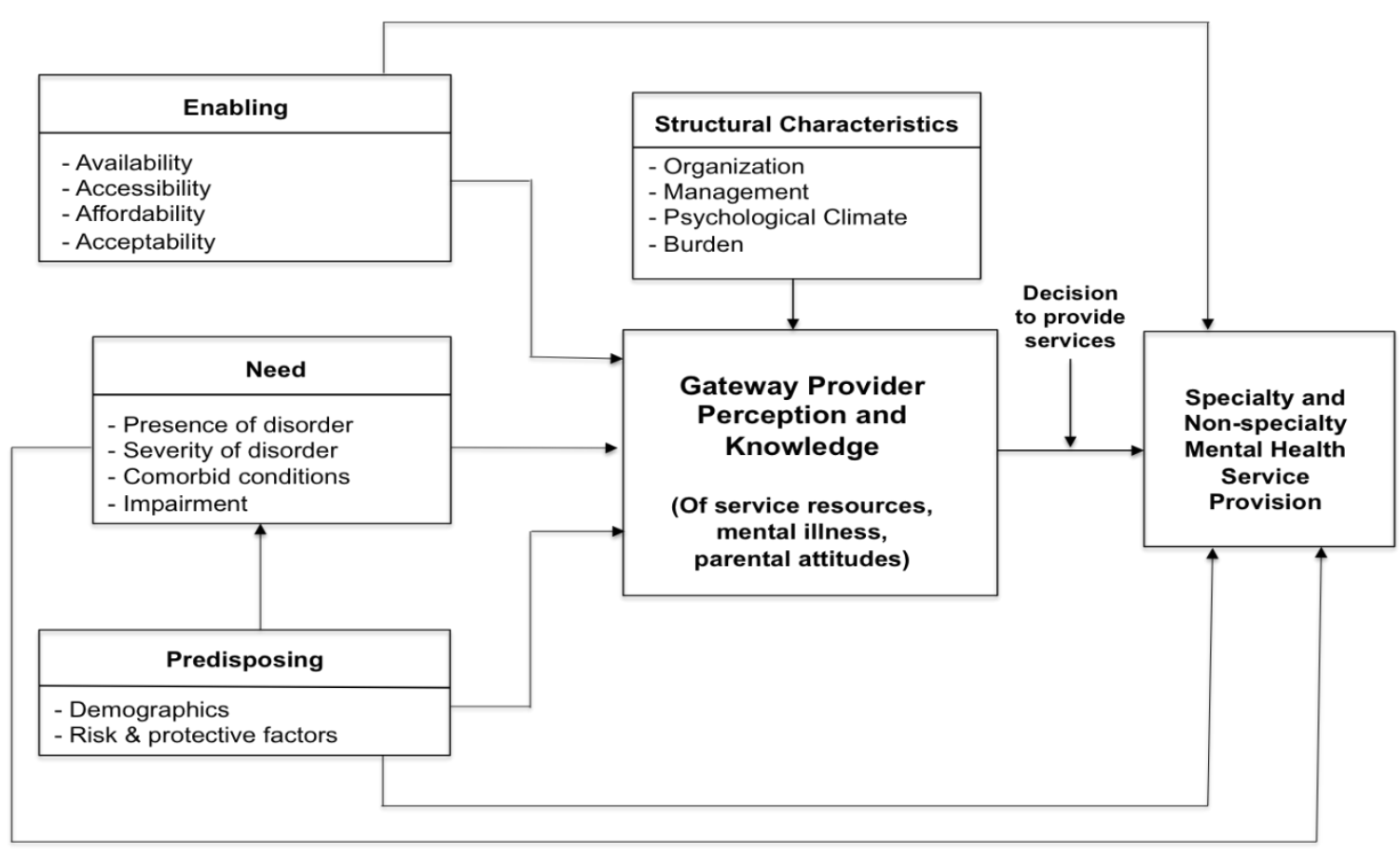

Figure 2-1 Gateway Provider Service Framework (Stiffman et al., 2004).

\subsubsection{Behavioral Model}

This model is used in GPM to explain what factors determine peoples' use of health service (Andersen, 1995). They are typically conceptualized as client variables (Stiffman et al., 2001). Predisposing characteristics include demographics and risk and protective factors such as neighborhood factors (e.g., negative neighborhood or violence exposure) and support system (e.g., family and peer environment). In addition, enabling characteristics include availability, affordability, and self-efficacy for obtaining services, 
and need characteristics include health status (diagnoses or symptoms) and functional impairment (Calsyn \& Roades, 1993). By putting Andersen's model in GPM, it explains the process or reason why clients decide to use services (Andersen, 1995). Enabling, need, and predisposing variables in Figure 2-1 are explained by this model.

\subsubsection{Network Episode Model (NEM)}

This model focuses on factors related to decision-making in health care. In the model, the provider's decisions are made within the context of interpersonal interactions, especially in one's social network (Edmonds, Hruschka, Bernard, \& Sibley, 2012). In other words, decision-making is related to interactions that are shaped by network structure and content as opposed to decision-making based on a client's needs (Kincaid, 2004). Pescosolido (1992) suggested that the treatment system related to children's mental health or drug problems produced treatment networks based on the structures' culture and mood. This turns service provision into a treatment decision that may or may not be the best option for the client.

During this process, the knowledge, experience, or resources of the gateway provider, built up through networks or interactions, may reflect on the service provision for clients; it does not necessarily guarantee that the best resource or service is provided. As a result of social interactions, providers may respond by drawing on familiar habits or organizational routines rather than by considering the client's unique needs (Stiffman, Pescosolido, \& Cabassa, 2004). Therefore, the NEM focuses on how service providers' network affects their decision and how they act or behave with their clients.

\subsubsection{Decision Theory}

While the NEM is in charge of interface factors that affect service provision, 
Decision Theory is the theory of rational decision-making, and it plays a role in how service providers choose the appropriate service provision. The aim of Decision Theory is to make rational decisions as accurately as possible (Peterson, 2009). A rational decision is one that the decision-maker chooses based on all available information and every possible outcome at the time when the decision is made (Peterson, 2009). Therefore, Decision Theory attempts to define how to choose the best outcome when faced with several options (Resnik, 1987). Gateway providers are frequently confronted with a set of options when they meet individuals in need and must balance personal and organizational influences with all possible outcomes in a situation.

A decision that needs to be made differs from one agency context to another. Consequently, work environments with no time constraints and easy workloads are more likely to result in accurately analyzed situations than burdened work environments in which decisions need to be made quickly (van de Luitgaarden, 2009). At the same time, a decision could differ between/amongst individual workers in the same environment, depending on the degree to which they employ some kind of organizing principle. This demonstrates that because there are a multitude of criteria to consider, service providers cannot make a decision without applying certain rules or values (van de Luitgaarden, 2009). Even if the service providers know which services should be used, it can often be difficult to ensure the correct services are employed.

Based on the key points of the NEM and Decision Theory, the GPM focuses on three elements that affect service provision (Stiffman, Pescosolido, \& Cabassa, 2004). First, it incorporates the NEM concepts as they relate to the individual. The person in contact with clients assesses the client's needs, determines service access for the client, 
and provides referral to other services through interaction with the provider's network of connections. These are all key roles of the individual as a gateway provider. Second, it incorporates Decision Theory concepts, which is influenced by the amount of information available. If service providers have access to more information, they can offer and refer better service to clients. Lastly, the service provider's attitude, their impression of the support available for treatment, and an overloaded system are some factors that might affect the service quality. An adaptation of the GPM provides the conceptual foundation for this study to examine the predictors of service provision by refugee service providers.

In a previous study using the Gateway Provider Model, the service providers' environment heavily affected their decision (as cited in Stiffman, Pescosolido, \& Cabassa, 2004, p. 6), as did the relationship between the worker and the organization. However, the relationship between organizational structure and systemic characteristics in the service providers' working environment, and the service provision has not been clarified because the GPM mainly focuses on the role of service providers. Even if service provision to clients is not good, the service could be provided in a good way, depending on the service providers' capabilities.

\subsection{Cultural Competence Model (CCM)}

The concept of cultural competence has been used for a long time in the social sciences to better understand and address social, health, and educational services to individuals in a culturally diverse society (Simpkins \& Riggs, 2014). The latest Census data have revealed significant increases in minority populations across the United States (Kunkle, 2015). Non-Hispanic, single-race Whites were the only group for which deaths 
exceeded births between 2013 and 2014. Additionally, minority children younger than five now make up 50.2 percent of that population group (Frey, 2015). It shows that the nation is becoming more diverse and means we possibly meet with people from various ethnic or cultural backgrounds more than before. Hence, the issue of cultural competence has been broadly used in many fields.

Before discussing the definition of cultural competence, "culture" and "competence" could be thought of separately. The word "culture" implies the integrated pattern of human behavior that includes thoughts, customs, values, and institutional belief (Cross et al., 1989; National Association of Social Workers [NASW], 2000). And, it shares and holds a group of people together and distinguishes them from other groups (Salimbene, 1999). Understanding the impact of culture on human service provision is a fundamental step to implementing service provision for various ethnic groups. The word "competence" means the capacity to function effectively (U.S. Department of Health and Human Services, Office of Minority Health, 2002) and perform specific tasks or skills necessary to achieve a particular goal (Bandura, 1986).

Based on the above definitions, cultural competence could be defined as "the ability of individuals to establish effective interpersonal and working relationships that supersede cultural differences" (Cooper \& Roter, 2002, p.554). Moreover, cultural competence is "a set of congruent behaviours, attitudes, and policies that come together in a system, agency or among professionals and enables that system, agency or those professionals to work effectively in cross cultural situations" (Cross et al., 1989, p. 13). Finally, according to the National Association of Social Workers (NASW), "cultural competence refers to the process by which individuals and systems respond respectfully 
and effectively to people of all cultures, languages, classes, races, ethnic backgrounds, religions, and other diversity factors" (NASW, 2015, p. 13). Regarding the above definitions, cultural competence is never fully realized, achieved, or completed, but rather cultural competence is a lifelong, ongoing and continuous process to develop capacity in order to provide effective service within the client's cultural context (Campinha-Bacote, 2002; Giger \& Davidhizar, 2013; Jeffreys, 2010; NASW, 2001; Smith, 1998; SuarezBalcazar \& Rodakowski, 2007; Suh, 2004).

Therefore, the person who is culturally competent would have diverse values, developed adaptations to diversity, institutional cultural knowledge, the capacity for cultural self-assessment, and an awareness of the dynamics inherent when cultures interact (Cross et al., 1989). Based on this definition, this study assumes that providers who are culturally competent, thus providing service comfortably to clients, have a higher capacity of cultural competence to provide service than those who are less culturally competent.

Several studies identify key elements as well as instruments developed to measure cultural competency. In Shen's (2015) literature review study, he reviewed cultural competency models (CCMs) and assessment instrument research published by nurse researchers since 1982. His review analyzed the concepts of cultural competence in each study and identified four attributes that the literature demonstrated most consistently: sensitivity (knowing cultural differences and similarities), awareness (one's own cultural and professional background), knowledge (the process of seeking and obtaining information about diverse cultures), and skill (the ability to assess cultural issues). According to Beach et al.'s (2005) cultural competence systematic review study, their 
research demonstrated cultural competence as a combination of provider knowledge, provider attitude, and provider skills. Rooda (1992) studied cultural competence among assigned nurses of the three cultural groups (Black American, Hispanic, and Asian American). The study used the concepts of cultural knowledge and awareness to see cultural competence. In addition, Campinha-Bacote (2002) developed the Inventory to Assess Cultural Competence among Health Care Professionals (IAPCC). She used five domains: cultural awareness, cultural knowledge, cultural skill, cultural encounters, and cultural desire. All five constructs have an interdependent relationship with each other. Therefore, the health care providers should work to improve the balance of all five. The other study that identified the key concepts for the CCM includes demonstrated cultural diversity, cultural awareness and sensitivity, and cultural competence behaviors (Schim, Doorenbos, \& Borse, 2005). Using these components, the study examined service providers in a hospice setting. Starr and Wallace (2009) studied cultural competence of public health nurses (PHNs) using cultural awareness and sensitivity, cultural competence behavior, and diversity experience. They found that nurses have moderate competence in thought (awareness and sensitivity), but this did not show in a comparable level of behaviors. That is because there are several obstacles to providing better service to clients, such as language barriers or lack of interpreters.

There are varieties of core components that have been identified by researchers, but cultural awareness (knowledge) and sensitivity (attitude), and cultural behavior have been used in the majority of cultural competence studies as key components of CCM. Those key components are (a) cultural awareness and sensitivity, which are based on one's cultural knowledge, are used to develop a critical view of cultural differences and 
then recognize one's own cultural biases; and (b) cultural behavior is everyday practice learned from personal exposure and experience with people from diverse ethnicities (Schim, Doorenbos, \& Borse, 2005; Suarez-Balcazar et al., 2011).

Therefore, this study used the cultural competence framework (See Figure 2-2) to know service providers' cultural competence in both thought and action.

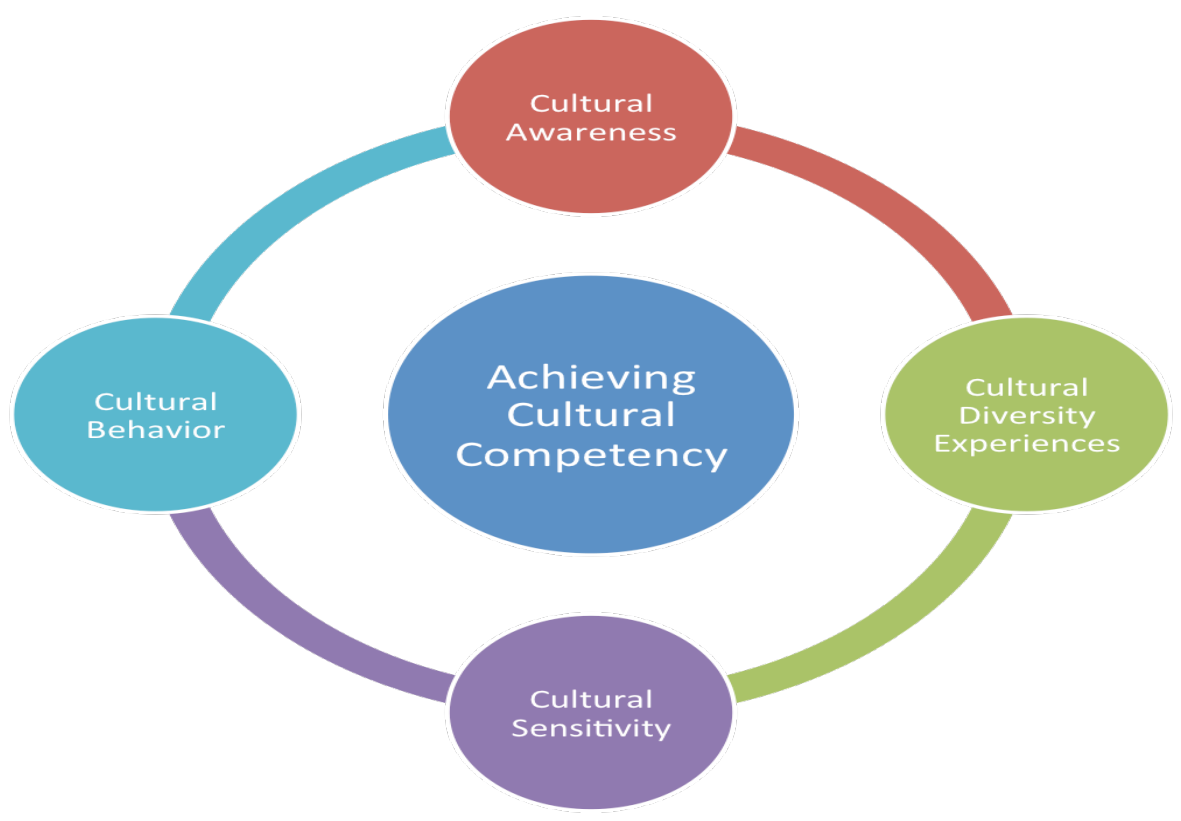

Figure 2-2 Contextual Model of Cultural Competence

\subsection{Organization Theory: Organizational Culture}

Organization theory was based on the efficiency of industrial production

processes (Glisson et al., 2008b), and it can help us understand what organizations are and how they behave in a certain organizational environment (Hodge \& Anthony, 1988). The most important early contributor was Max Weber, who emphasized bureaucracy as the ideal type of organization (Starbuck, 2003). The early studies, including Weber's studies, were centered on the organization itself rather than on the 
relationship of the organization to its surrounding environment (Hodge \& Anthony, 1988).

The classical school, the formal school of management thought, on the other hand, believed that the organization would do better when focused on determining rational approaches and techniques whenever possible (Forbes, 2012). Frederick Taylor is an important contributor among them (Hodge \& Anthony, 1988). The basic assumption of his study is that employees perform their best and work most efficiently when the organization has control (Taylor, 1913). Therefore, he emphasized "one best way" to perform a task and to make that way standard practice. That means if managers applied the best practice principle, they would proactively improve the quality of management and it would provide benefits to their organization as a whole (Hodge \& Anthony, 1988). These Classicists concentrated on how to carry out work in the most efficient manner. However, it was too narrow of a view and ignored how the human element should act in an organization. Humans have abilities to do something, and this could affect the production of the organization.

The behavioral school was a group that was concerned with the human element of an organization (Hodge \& Anthony, 1988). This group aimed to understand people's behaviors, such as group norms and customs, for organizational effectiveness (Hodge \& Anthony, 1988). Behaviorists argued that work condition, participation, cooperation, communication, leadership, and human motivation were important factors for the way that person behaves in an organization (Clegg, Kornberger, \& Pitsis, 2011). McGregor's Theory $\mathrm{X}$ and $\mathrm{Y}$ is a most notable study of human behavior (Hodge \& Anthony, 1988). The Theory $\mathrm{X}$ assumption is that employees dislike and attempt to avoid work, 
need direction, avoid responsibility, and lack ambition. In contrast, the Theory Y assumption is that employees do not dislike work, have self-control and direction, and seek responsibility (McGregor, 1960). Current thinking is that a positive view of workers, Theory $\mathrm{Y}$, is acceptable and results in more success than Theory $\mathrm{X}$ to improve organizational effectiveness (Kopelman, Prottas, \& Davis, 2008). Therefore, the view of human behavior in organizational theory is that awareness of the drive for individual selffulfillment led companies to take a long look at how their employees were managed, motivated, and led (Carson, 2005). The behavioral school focuses on human relationships in an organization and the system theory approach focuses on the relationships, not only examining how component parts of the organization function together and interact inside, but also how organizations interact with their surrounding environment (Hodge \& Anthony, 1988). This approach is macro-level, thus it allows for detailed analysis of organizational components such as human relationships, technologies, or structural dimensions (Kast \& Rosenzweig, 1996).

There are several types of organizational theories that could explain organization structure and effectiveness. The concepts are different but have one thing in common: the character of the organization could affect workers and their outcomes. This similarity indicates that there are limitations on outcome efficiency and individual effectiveness (Glisson et al., 2008a). This is because the organizational social situation is the total set of outside forces surrounding and shaping the behavior of the organization and it could effect a member's performance in many ways (Ehrhart, Schneider, \& Macey, 2014). An organizational view can provide insights into the environment in which an organization functions and how that influences people in that work environment (Murray 
\& Holmes, 2012). To explain those relationships, organizational culture conceptualizes the way people experience and describe their work setting (Schneider, Ehrhart, \& Macey, 2013). The concept of organizational culture is important because it pervades all aspects of an organization's operations. It affects how goals are determined and how work is conducted (Hodge \& Anthony, 1988). A general definition of organizational culture is "a system of shared values and beliefs that produces norms of behavior and establishes an organizational way of life" (Koberg \& Chusmir, 1987, p. 397). Further, Pettigrew (1979) defined organizational culture as a combination of the ideology, beliefs, and myths held by members of an organization. He described that understanding an organization's culture was necessary for members to make sense of their everyday work. Organizational culture is concerned with the organizational system and deep structure of the organization. Moreover, organizational culture is an organizational-level construct assessed as the behavioral expectations that members of an organization are required to meet in their work environment (Verbeke, Volgering, \& Hessels, 1998). Standards could be provided to workers for what to do and how to act (Sackmann, 1991). These expectations are priorities that are most important to the organization. For example, refugee resettlement service centers expect that service providers will maintain culturally competent skills and they try to promote that refugee quality of life is the highest priority. It could be an organizational culture for refugee resettlement service centers.

There are several components of organizational culture, according to Delobbe, Haccoun, and Vandenberghe (2002). In their research paper on organizational culture, they indicated that the most common dimensions of organizational culture are innovation (resistance), control (rigidity), and results/outcome (proficiency) orientation based on 
analyzed organizational culture questionnaires. Innovation indicates general openness to change. The organization expects that people in the organization have a desire to change, which could be an innovative organizational culture. The control dimension focuses on the level of work formalization, the existence of rules and regulations, and the importance of the hierarchy (Delobbe, Haccoun, \& Vandenberghe, 2002). The organization expects that people in the organization report all decisions to a supervisor, which could be a controlled organizational culture. Lastly, result/outcome-oriented culture indicates that it measures the level of productivity (Delobbe, Haccoun, \& Vandenberghe, 2002). This culture refers to workers' skills to enhance performance and satisfaction of clients.

Therefore, one of the most influential factors on service providers' decision behavior is the organizational culture in which they work (Glisson, 2002). Thus, even if service providers are culturally competent or have the knowledge and skills, they work within restrictions regarding their possibilities of providing services. These variations in cultures across the research point to the utility of examining how social context variations might relate to refugee service provision.

Understanding the role of organizational factors in a social service agency might lead to better ways of providing service to refugees. For the purpose of this study, organizational culture is a variable of organizational factor. It can help explain the way organizational characteristics reflect and affect the environment in which service providers exist. Therefore, organizational culture provides important conceptual and empirical bases for understanding the organizational social context.

\subsection{Conceptual Model of the Study}


The Gateway Provider Model has not been previously applied to health service providers, especially service providers for refugees. The GPM applied to service providers for refugees is a major theoretical framework of this study. Moreover, adding two models/theories - CCM and organizational theory—-this study holistically examined the importance of the roles of service providers who provide care/service to clients with diverse values (Aragaw, Yigzaw, Tetemke, \& G/Amlak, 2015).

Refugees, one of the most vulnerable population groups, have unique challenges such as resettling and acculturating into a new country with a language barrier. CCM explains the importance of a proper attitude toward refugees. When a refugee's culture is not understood, inappropriate services could be provided. Furthermore, the work environment of service providers could be one of the crucial predictors of providing services. Based on the organizational environment, service providers might come to different decisions. Those three models/theories will help to explain gaps and bridge between service providers and service provision. Thus, using aspects of the GPM (personal factors), $\mathrm{CCM}$ (personal factor), and organizational theory (organizational culture factors) helped to examine what factors affect service provision for refugees.

\subsection{Literature Review of Empirical Research}

A literature review of studies related to service provision helped to specify which factors to include in the conceptual model of this study, which is specific to service provision for refugees. The factors of interest are the service provider's personal factors related to service provision and organizational culture factors related to service provision.

\subsubsection{Personal Factors and Service Provision}


Findings from previous studies show that service providers' personal factors impact service provision. There are several personal factors relating to service provision: knowledge of the clients (refugees), familiarity with community resources, and cultural competence. Yet, little is known about how social service providers' personal factors are related to service provision, especially for refugees. Therefore, in this part, reviewed literature includes general service provider studies.

Knowledge of Clients (Refugees). Service providers try to understand and should have knowledge about their target population for better service provision (Copeland, 2006). Moe and Sparkman (2015) identified service providers' barriers to service access experienced by their clients at lesbian, gay, bisexual, transgender, and questioning (LGBTQ)-affirming social service agencies. They found when the providers have more LGBTQ knowledge, they better identify and understand their clients and their barriers to accessing service, and it helps them provide appropriate services to their clients. Another study done by Olivier and Dykeman (2003) identified challenges of HIV service provision among nurses and social workers who had provided HIV-related services. They found that one of the biggest barriers to service is the provider's lack of HIV knowledge. Moreover, service providers who participated in this study strongly endorsed the importance of HIV education for better service provision. In a study that examined service provider factors that affect service provision for individuals with intermittent preventive treatment for malaria in pregnancy (IPTp) in Nigeria (Onoka, Onwujekwe, Hanson, \& Uzochukwu, 2012), they found that there was a low level of knowledge of IPTp by all providers and this had negative effects that can lead to ineffectiveness of service provision. 
To my knowledge, there are no studies on refugee providers' refugee knowledge and service delivery, although there has been some research investigating refugees' access to health care services (Kirmayer et al., 2011; Steel et al., 2011; Sundvall, Tidemalm, Titelman, Runeson, \& Bäärnhielm, 2015). One research study by Weine, Kuc, Dzudza, Razzano, and Pavkovic (2001) explored the relationship between refugee service provider's knowledge (refugee PTSD) and their mental health service provision among service providers who were working with Bosnian refugees. They found that the provider's own knowledge of refugee trauma would be expected to play an important role in delivering refugee mental health services. No service provider can provide improved interventions without understanding the refugee.

Familiarity with Community Resources. Bunger, Stiffman, Foster, and Shi's (2009) study examined the importance of child welfare workers' familiarity with community resources for youth at high risk for mental illness, substance abuse, and other behavioral health issues. They found that youth received a greater variety of mental health service when their service providers were familiar with other community resources. Another study predicted factors of addictions and mental health services provided to American-Indian youth. Providers were more likely to identify a youth's problems and refer services when they had more knowledge of community resources and American Indian youth (Stiffman et al., 2006). In addition, if the provider's knowledge of mental health resources can be enhanced, provider ability to offer service will increase (Stiffman et al., 2001). Copeland (2006) focused on increasing providers' knowledge of the sociocultural factors that influence mental health service utilization for African American adolescents. This study suggested the service provider must have awareness of 
mental health services and knowledge about resources to provide better service. In addition, Nalwadda and colleague (2011) conducted a sexual health study related to pregnancy prevention service provision. Through semi-structured interviews with providers (medical officer, clinical officer, nurse, and nursing assistant) in Uganda, they focused on factors that influenced providing contraceptive service. Lack of knowledge and a low level of competence regarding contraceptive service, such as comprehensive counseling, are the biggest factors influencing service provision.

There are few studies that examine service providers' work with immigrants and refugees. Tastsoglou, Abidi, Brigham, and Lange (2014) conducted a qualitative study on experiences and perception of immigrant service providers in relation to women refugee claimants in Atlantic Canada. They found that there is a lack of services and resources to support clients, thus providers have difficulties in navigating services. Moreover, a systematic review of health service for migrants was conducted to identify how providers' individual attitude, work place factors, and external environment influenced service provision (Suphanchaimat, Kantamaturapoj, Putthasri, \& Prakongsai, 2015). Most of the reviewed research concluded that there is a lack of human and institutional resources that limits providing health services to migrants. Another systematic review of undocumented people by Drachman (1995) emphasized service providers' need for familiarity with resources that exist outside the mainstream of the public system. Jewson and colleagues (2015) interviewed refugee service providers in the Geelong region of Australia to explore their perceptions of capacity of service providers and services to enhance the health and well-being needs of refugees. The findings revealed that a more 
coordinated approach would increase the effectiveness of existing services; however, current resource levels could not meet refugee needs.

Cultural Competence. Service providers should be culturally competent because culturally relevant service rests on understanding the clients' cultural worldview, communication patterns, family dynamic, and behavior (Leigh, 1998). Therefore, cultural competence is an important aspect in human service where providers assist diverse populations.

Many studies emphasize the importance of service providers' cultural competence in healthcare settings. This might be because sometimes health care professionals deal with life-saving information (Van Keer, Deschepper, Francke, Huyghens, \& Bilsen, 2015). The following is an example from the South Cove Community Health Center: "When South Cove providers advise parents to give cough syrup to their children, they must remember that Chinese 'teaspoons' are generally much larger than American ones. Without this information, many children would be dangerously over-medicated" (Health Resources and Services Administration, 2001, p. 15). In addition, there are health outcome disparities because of service providers' lack of cultural competence in service provision. Smedly, Stith, and Nelson (2002) indicate that racial and ethnic minorities are less likely to receive medical services from service providers than White Americans. This issue has led to disparities between health service provision of minority groups and the majority population (Taylor, 2005). In addition, Betancourt, Green, Carrillo, and Park (2005) highlighted key perspectives and trends to improve and eliminate racial/ethnic disparities in health care. The authors conducted interviews with 37 experts. They used a structured interview guide with ten open-ended questions and, based on the results from 
the interviews, identified themes according to frequency and relevance. They found that cultural competence might improve outcomes and help control costs by making care more effective and efficient.

And, yet, few studies have been conducted on the role of cultural competence in service providers for refugees or immigrant service providers and service outcomes. Tastsoglou, Abidi, Brigham, and Lange (2014) found the service providers who have a low level of cultural competence provided inappropriate services to refugee women. Women in some refugee groups need special care, such as female staff, but the service providers in this study did not have this cultural information; thus, services were not provided effectively. Moreover, inefficient interpreter services negatively affected communication and it decreased effective service provision. The qualitative study of Griswold, Zayas, and Kernan (2007) was to determine if cultural awareness lessons were needed for medical students providing service to refugee patients. In their experiences, the students found that they needed cultural training such as interpretation service, information about the refugee's cultural background, and understanding of cultural humility to build up cultural competence for better service. Håkonsen, Lees, and Toverud (2014) studied the view of pharmacists and the obstacles of non-Western immigrants when they used drugs in Norway. Health providers found it challenging to provide adequate service to clients due to language and cultural barriers. Thus, the level of cultural competence of refugee service providers may relate to service provision.

\subsubsection{Organizational Culture Factors and Service Provision}

The organization environment is the overall atmosphere in which the service providers work. It includes the organizations' norms, values, and expectations of 
workers, as well as members' perceptions of the organization, all of which affect how services are provided (Glisson, 2007). Those organizational factors could determine what the priorities are in the work environment or the limitations of available resources. Frontline providers feel that most of the organizational conditions affecting quality of service are beyond their control (Yazdi-Feyzabadi, Emami, \& Mehrolhassani, 2015). Thus, the individual service providers who work there believe they should follow their agency roles. Therefore, organizational factors might effect a service provider's service provision positively or negatively. Extensive research has focused on the influences of organizational culture factors on service provision.

Organizational Culture. Many studies have demonstrated that organizational culture, as an organizational factor, affects service implementation and quality (Aarons \& Sawitzky, 2006; Brunette et al., 2008; Glisson, 2007; Glisson \& Green, 2006; Glisson et al., 2008a; Guzzo, Jette, \& Katzell, 1985; Parker et al., 2003). This was shown to be the case in a Glisson, Williams, Green, Hemmelgarn, and Hoagwood (2014) study where the Organizational Social Context measure was administered on-site to 209 family support specialists and clinicians in 21 mental health programs in New York State. Additionally, US regional and nationwide studies showing that children served by child welfare agencies with a positive organizational culture environment, such as service providers willing to place the wellbeing of the client first and have up-to-date knowledge have better clinical and functional outcomes than children served by agencies with a negative agency cultural environment (Glisson, 2010; Glisson \& Green, 2006; Glisson \& James, 2002). The role of the organizational culture in individuals' work behaviors has been researched (Aarons et al., 2012). Glisson and colleague (2008a) presented a study 
showing that organizational characteristics, culture, and climate relate to the successful implementation of mental health services. They found that organizations with the best culture sustain new treatment or service programs over twice as long as organizations with the worst cultures. Therefore, if organizations care about their work culture, service quality and service outcome could be improved. Another organizational factor and service outcome study showed that cultural factors (i.e., shared behavioral norms, work priorities, and expectations of front-line service providers) within organizations have a notable influence on service provision (Olin et al., 2013).

Once again, organizational culture factors may be positively or negatively related to the service provider's service provision. It could be found that even though there is a great willingness to engage more with refugee clients and provide services, the structural and organizational environment could make the service provider ineffective.

\subsection{Gaps and Summary}

Overall, the literature review reveals the key studies that have examined important predictors of service provision for refugees, such as the personal factors and organizational culture factors of the service providers. Moreover, despite the rapid expansion of research on refugees over the last decade, the challenges in understanding the service provider have not been greater. Several gaps still exist in our knowledge about service providers' service provision for refugees. As described below, the current study addresses some of the critical gaps in the literature and examines hypotheses regarding key factors related to service provision by refugee service providers. 
First, most of the studies have been focused on the refugees. Many studies have examined issues related to refugee life. Several of them have dealt with topics about the difficulties and importance of refugee resettlement and adjustment (Bayram, Thorburn, Demirhan, \& Bilgel, 2007; Birman, Trickett, \& Vinokurov, 2002; Schweitzer, et al., 2006; Silove, Sinnerbrink, Field, Manicavasagar, \& Steel, 1997; Terheggen, Stroebe, \& Kleber, 2001) and many researchers have been interested in the refugee acculturation process and stress (Beiser \& Hou, 2001; Kang, 2006; Kayes \& Kane, 2004; Kiteki, 2011; Miller et al., 2002; Mollica, Sarajlic, Chernoff, \& Lavelle, 2001; Sabbah, 2007; Sam \& Berry, 1995; Silove, 1999; Tran, 1992; Yoon, Lee, \& Goh, 2008). The most popular topic in studies about refugees has been refugees' health issues; several researchers have examined the mental and physical health of refugees (Araya, Chotai, Komproe, \& de Jong, 2007; Beiser, 2005; Carlsson, Mortensen, \& Kastrup, 2006; Fox \& Tang, 2000; Lavik, Hauff, Skrondal, \& Solberg, 1996; Mann \& Fazil, 2006; Procter, 2005; Pumariega, Rothe, \& Pumariega, 2005; Watters, 2001). Very few studies have examined service providers, yet their roles, challenges, barriers, and needs impact their ability to address the issues of their clients. It is absolutely necessary to understand and pay attention to the life of refugees. However, how service providers deliver appropriate services to refugees is also important for the successful resettlement of the refugees. Therefore, exploring the issues of service providers who are in charge of delivering services will lead to better service outcomes.

As mentioned, there are very few studies about social service providers. Most studies have targeted health professionals to try to determine the important factors for optimizing service outcomes (de Figueiredo, Yetwin, Sherer, Radzik, \& Iverson, 2014; 
Nalwadda et al., 2011; Olivier \& Dykeman, 2003). Other service providers who work with refugee populations have been overlooked. Several studies have dealt with service providers' perceptions and needs when providing services to specific populations, such as LGBTQ (Moe \& Sparkman, 2015), African American adolescents (Copeland, 2006), and children with a high risk for mental illness (Bunger, Stiffman, Foster, \& Shi, 2009).

Service providers might have different approaches to service provision depending on the service population.

The qualitative research method has been used by most researchers to understand the service providers' individual experiences when they provide services to refugees (Jewson et al., 2015; Johnson, Ziersch, \& Burgess, 2008; Nalwadda et al., 2011; Onoka et al., 2012; Tastsoglou et al., 2014; Weine et al., 2001). In-depth and semi-structured interviews and focus groups have been shown to be helpful for gaining insight into the ground-level perceptions and experiences of service providers. These studies have provided unique and detailed information. However, given the small number of participants drawn from only specific cities, these studies may not adequately represent the population of providers working with refugees, and thus these results cannot be generalized. Therefore, to discover the general predictors and issues regarding service providers who work with refugees, a larger number of participants from a higher number of programs in different cities are needed.

There is very little empirical research related to the providers' cultural competence while providing service to refugees. In contrast, there are many studies on the importance of culture in social services, especially in healthcare, as well as articles that emphasize the importance of the cultural competence of service providers (Aragaw, 
Yigzaw, Tetemke, \& G/Amlak, 2015; Betancourt \& Green, 2010; Larsen \& Reif, 2011; Suurmond, Seeleman, Rupp, Goosen, \& Stronks, 2010). However, the importance of cultural competence itself and the importance of the level of service providers' cultural competence in healthcare is overrepresented while they are underrepresented in general social services. Healthcare services are needed for refugees, but so are other services. Thus, cultural competence should be integrated into all aspects of social services rather than be viewed as a specific service area in itself.

Organizational culture factors could be important predictors of whether a service provider enhances or reduces service provision (Aarons et al., 2012; Glisson, 2007). Organizational culture refers to the rules and procedures providers must learn in order to be accepted within an organization (Sriramesh, Grunig, \& Buffington, 1992). Therefore, depending on the organizational culture that the service providers have, their service outcomes could vary. However, the literature review did not reveal any studies that focused on the impact of organizational culture factors on service providers working with refugees. Most of the organizational culture studies were conducted only on mental health service providers (Glisson \& Green, 2006; Glisson et al., 2008a) or family support service providers (Olin et al., 2013). Therefore, this study suggests approaches that focus on understanding the organizational culture for all service providers.

There is a clear need for a greater understanding of service providers who are in regular contact with refugees and how their experiences play out in the service provision. It is important to identify the factors that affect the way service providers work with refugees in order to develop better quality and more appropriate services. Therefore, additional research is needed to understand the extent to which factors affect service 
providers' service provision. Chapter 3 explains the research design that addressed this issue, and the study results attempt to fill this gap.

\section{CHAPTER 3: RESEARCH DESIGN AND METHODS}

\subsection{Research Model}

Within a framework of the merged conceptual models/theories (the gateway provider model, cultural competence model, and organization theory), this dissertation proposes to utilize two research models as described below.

In designing the theoretical framework, the aforementioned studies helped to identify specific variables in each domain: a) personal factors including knowledge of clients (refugees; e,g., Copeland, 2006; Moe \& Sparkman, 2015), familiarity with community resources (e.g., Jewson et al., 2015; Tastsoglou, Abidi, Bringham, \& Lange, 2014), and cultural competence (e.g., Griswold, Zayas, \& Kernan, 2007; Tastsoglou et al., 2014); b) organizational culture factors including proficiency, rigidity, and resistance (Aarons \& Sawitzky, 2006; Glisson, 2007); and c) demographics of the service providers (i.e., age, biological sex, race/nationality, education, and job title). This study assumed that personal factors and organizational culture factors are related to service provision and referral for refugees.

\subsubsection{Research Model for Testing Direct Effects}

Figures 3-1 and 3-2, below, present a research model for testing direct associations of personal factors and organizational factors in predicting service provision and referral. This study hypothesizes that personal and organizational factors are directly associated with service provision for refugees. 


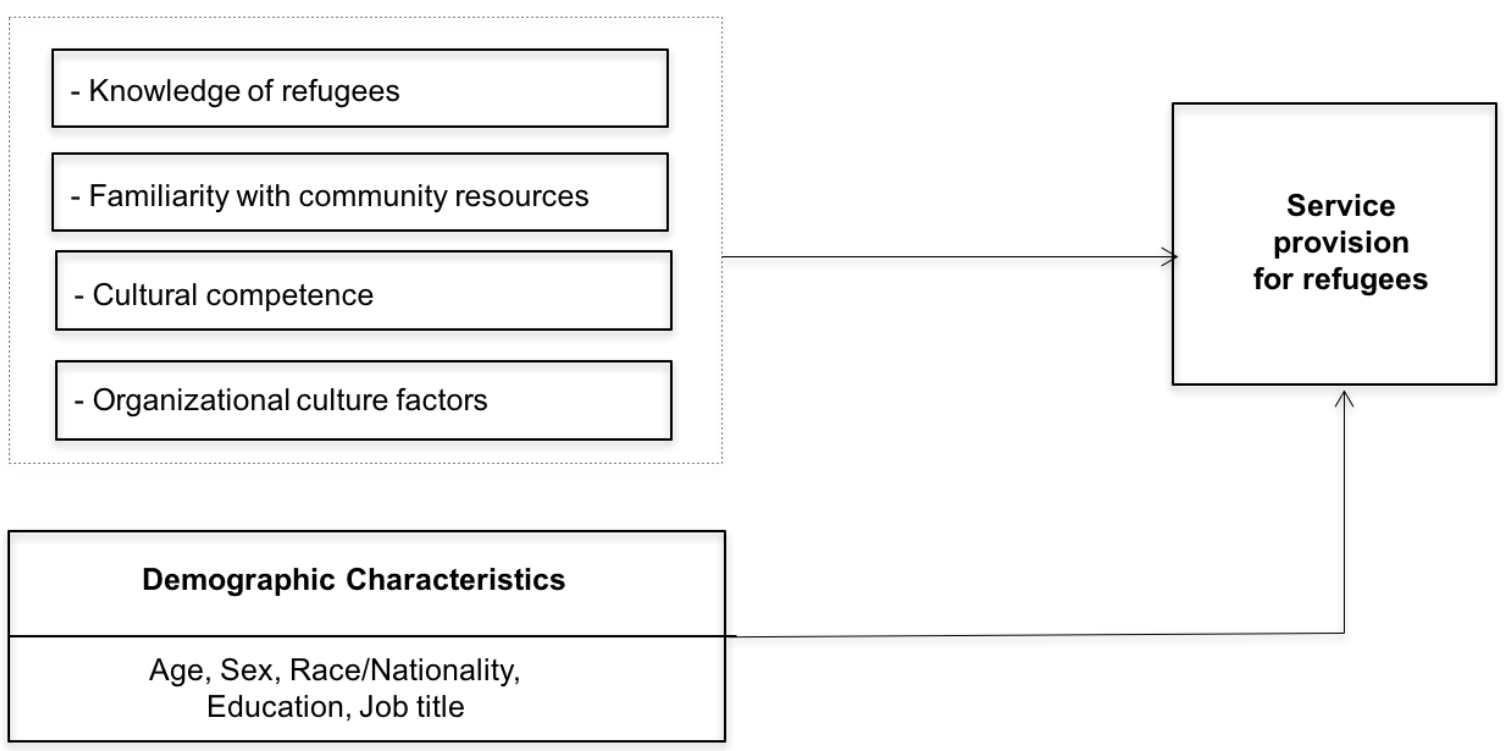

Figure 3-1 Research model for testing direct associations of multiple variables in predicting service provision

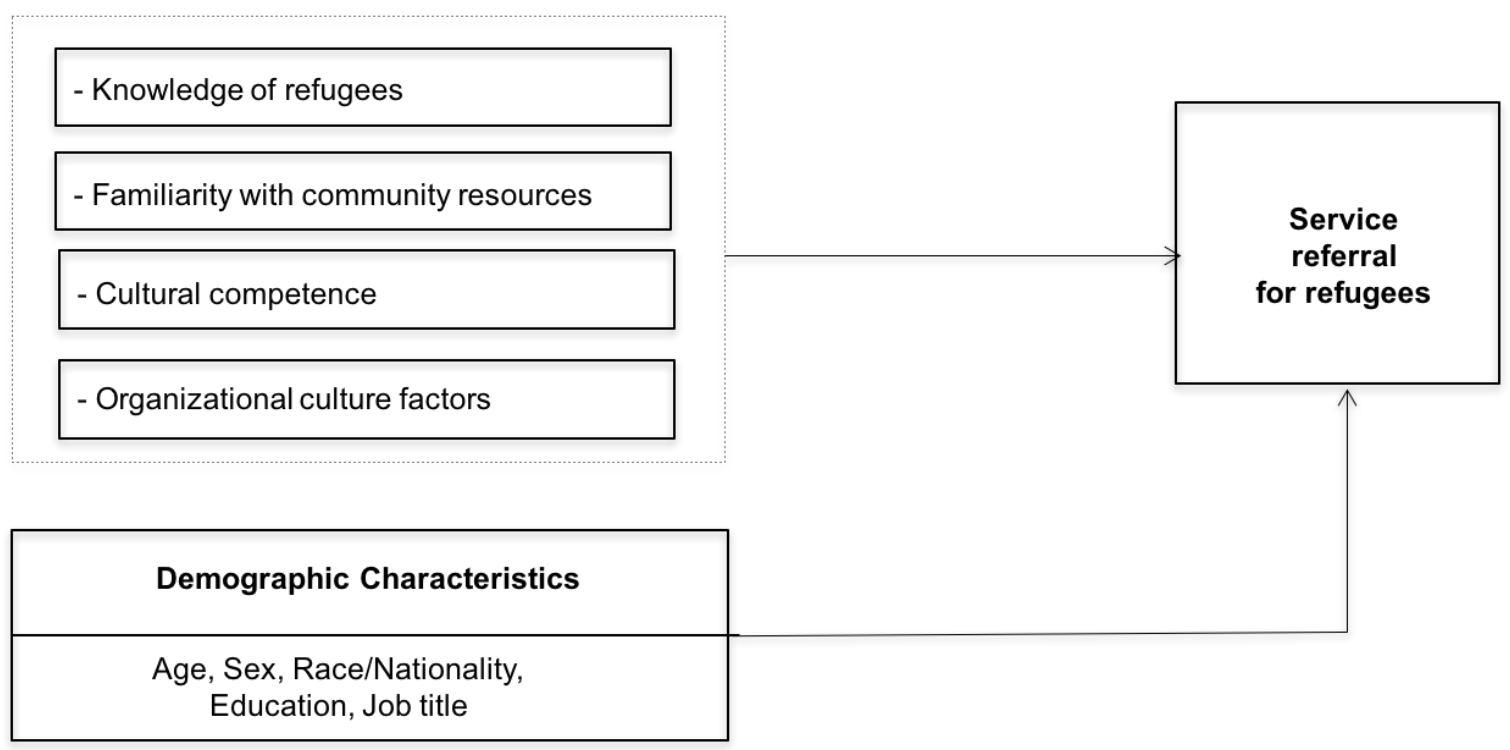

Figure 3-2 Research model for testing direct associations of multiple variables in predicting referral

\subsubsection{Research Model for Testing Indirect Effects}

Figures 3-3, below, present a research model for testing indirect effect of cultural competence associations of familiarity with community resources and work experience in 
predicting service provision and referral. This study hypothesizes that cultural competence behavior and cultural awareness/sensitivity are mediate association between familiarity with community resources and work experience and service provision/referrals for refugees.

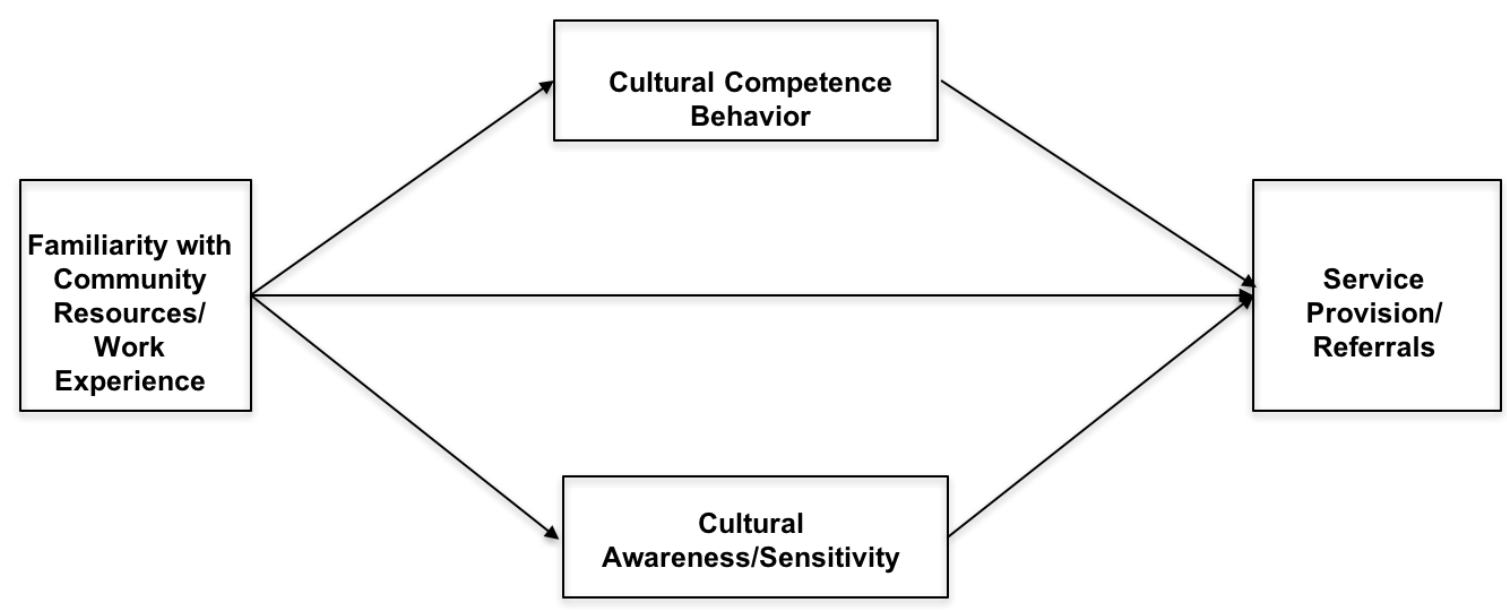

Figure 3-3 Research model for testing indirect associations of multiple variables in predicting referral

\subsection{Research Questions and Hypotheses}

Based on the three theories and review of the literature, this study specified the following research questions and hypotheses corresponding to the aims mentioned in Chapter 1.

Research Question 1: What types of services are currently being provided to meet the needs of refugees?

Research Question 2-1: What are the relative effects of personal factors (knowledge of refugees, familiarity with community resources, and cultural competence [cultural 
competence behavior and cultural awareness/sensitivity]) and organizational culture factors (proficiency, rigidity, and resistance) on service provision for refugees?

Hypothesis 2-1-1: Personal factors are stronger than organizational factors in predicting service provision for refugees.

Research Question 2-2: What are the relative effects of personal factor (knowledge of refugees, familiarity with community resources, and cultural competence [cultural competence behavior and cultural awareness/sensitivity]) and organizational culture facture (proficiency, rigidity, and resistance) on referral for refugees?

Hypothesis 2-2-1: Personal factors are stronger than organizational factors in predicting service referral for refugees.

Research Question 3: Does cultural competence behavior and cultural awareness/sensitivity mediate the associations between familiarity with community resources and service provision or referrals for refugees?

Hypothesis 3-1: Cultural competence behavior will positively mediate the association between familiarity with community resources and service provision for refugees.

Hypothesis 3-2: Cultural competence behavior will positively mediate the association between familiarity with community resources and service referral for refugees.

Hypothesis 3-3: Cultural awareness/sensitivity will positively mediate the association between familiarity with community resources and service provision 
for refugees.

Hypothesis 3-4: Cultural awareness/sensitivity will positively mediate the association between familiarity with community resources and service referral for refugees.

Research Question 4: Does cultural competence behavior and cultural awareness/sensitivity mediate the associations between work experience and service provision or referrals for refugees?

Hypothesis 4-1: Cultural competence behavior will positively mediate the association between work experience and service provision for refugees. Hypothesis 4-2: Cultural competence behavior will positively mediate the association between work experience and service referral for refugees.

Hypothesis 4-3: Cultural awareness/sensitivity will positively mediate the association between work experience and service provision for refugees. Hypothesis 4-4: Cultural awareness/sensitivity will positively mediate the association between work experience and service referral for refugees.

\subsection{Research Design}

The current study utilized a non-experimental, cross-sectional, quantitative design. An online survey was conducted using Qualtrics, which is a web-based survey tool. Additional details are provided below.

\subsection{Participant Recruitment and Data Collection Procedure}

Participants were recruited through the use of combined purposive and snowball 
sampling techniques, targeting social service agencies where a service is provided for refugees. This study had specific inclusion criteria: participants must have an employment (e.g., employment counselor, job developer, job specialist), education (e.g., English language instructor, ESL instructor, education specialist), health care (e.g., medical case manager, health coordinator, health counselor), or resettlement (e.g., case manager, social worker, resettlement coordinator, arrival coordinator, apartment setup coordinator, basic need specialist) related job title and have provided any service to refugees within the past 12 months. This was clearly stated on the recruitment email, consent form, and survey questionnaire. 


\section{Created recruitment list as a sample frame}

- Referred to the direction of state programs from the Office of Refugee Resettlement website as a sampling frame

- Checked the agencies that are providing a service to refugees

- Checked the service providers' inclusion job titles (employment, education, health, resettlement)

- Divided into four regions (Northeast, Midwest, South, and West)

- If there was no email contact information, the researcher called the agencies to ask for an email address if one could not be found

- Created a list of emails of potential participant

Sent the survey link to service providers who met the inclusion criteria

- Approached the South and Midwest agencies first

- Monitored the sample size from the South and Midwest Regions. Since the sample size did not reach 122 , reached out to the other two regions.

- Sent an email reminder at least three times at one-week interval to ask for survey completion

- Sent an email to agencies requesting that they share the survey link with other agencies meeting the participant criteria

- Sent out gift cards within 7 days of the survey completion

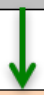

The data collection process was terminated when the sample size reached 122.

Figure 3-4 Data Collection Procedure

Figure 3-4 summarizes the data collection procedure. A recruitment list by state was created using the directory of programs from the Office of Refugee Resettlement website (http://www.acf.hhs.gov/orr/state-programs-annual-overview). The website is part of the U.S. Department of Health \& Human Services and provides comprehensive information on social service agencies for refugees. Refugee service agencies, service providers' contact information (particularly email addresses), and their job titles were identified. If there was no email address available on the website, those agencies were 
called to obtain contact information. The identified agencies were divided into four regions as defined by the U.S. Census Bureau (United States Census Bureau, n.d.; see Figure 3-5).

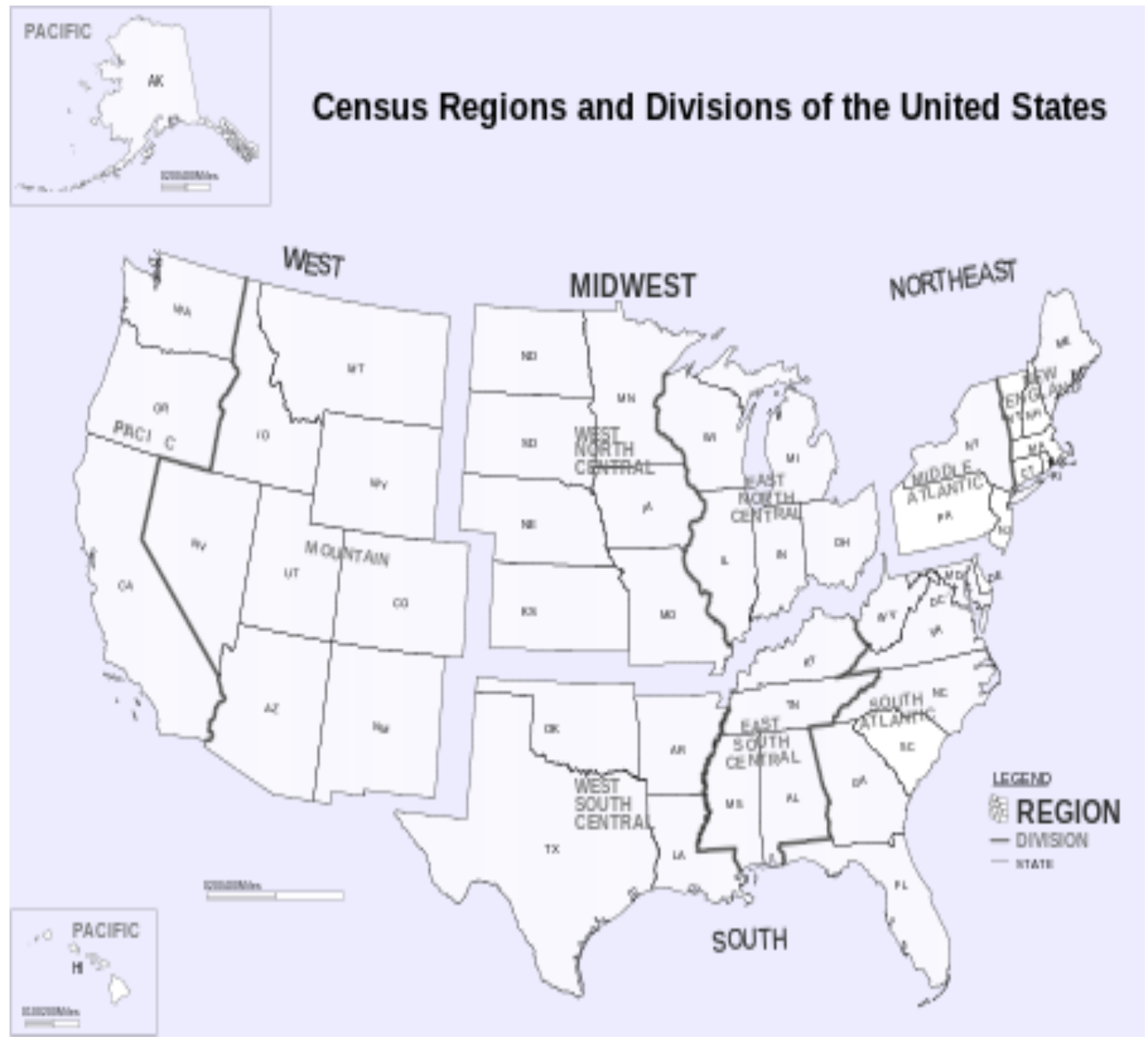

Figure 3-5 U.S. Census Bureau Regions

The four regions used within the survey are Northeast, Midwest, South, and West. Since the South and Midwest regions contain more refugee agencies (the Office of Refugee Resettlement, n.d.) and refugee arrivals than the other two regions (Department 
of State, 2016), data collection was started there (see Table 3-1). Accordingly, it was assumed that those two regions have more service providers and service provision for refugees. Data collection from the two regions did not reach to the target sample size, so data collection was extended to the other two regions.

Table 3-1 Number of Agencies and Refugee Arrivals by Region

\begin{tabular}{c|c|c} 
Region & Number of Agencies* & Number of Refugees* \\
\hline Northeast & 16 & 10,887 \\
\hline Midwest & 35 & 20,537 \\
\hline South & 34 & 22,094 \\
\hline West & 24 & 18,408 \\
\hline
\end{tabular}

* See Appendices A and B

The survey link was sent to service providers who met the inclusion criteria. Moreover, they were asked to share the survey link if they knew of other agencies meeting the inclusion criteria. It was anticipated that the link would be forwarded to other branches of the agencies or social service agencies that may have a high probability of working with refugees (i.e., refugee resettlement offices or health care centers). Reminder emails were sent at least three times at one-week intervals. Moreover, the service providers were requested to post the survey link on their Facebook or other social networking sites to increase the opportunity of reaching relevant refugee service providers.

Recruitment was also conducted by asking staff working for the Refugee and Immigration Services (RIS) office in Columbia, Missouri to send a survey link to other service providers. RIS is a service provided by Catholic Charities of Central and Northern Missouri and is one of the official resettlement services assisting refugees in the United States. Since it has its own network of service providers for refugees, this method was 
thought to be helpful. The data collection procedure ended when the planned sampling size of 122 was attained.

The survey took about 20 minutes to complete and participants received a \$5 gift certificate upon completion. If the participants wanted to receive a gift certificate and a summary of the results of the study upon its completion, they were asked to type their email or mailing addresses at the end of the survey. The email address was separated from the survey in order to maintain confidentiality.

For the protection of human subjects, this study obtained approval from the University of Missouri Campus Institutional Review Board (IRB). To guarantee respondents' confidentiality, no identifying information was collected through the survey and results were reported in aggregate. Through the consent form, respondents were informed that participation was voluntary and could be discontinued at any time. The consent form included a description of participant protections and rights.

Lastly, this study was fully funded by the University of Missouri School of Social Work's PhD program dissertation research award.

\subsection{Estimated Sample Size}

For a precise estimation of appropriate sample size, statistical consultation was requested. A power analysis was conducted using a SAS software program. The sample size was determined using a medium effect size, $80 \%$ power level, and significance level set at $\alpha=0.05$. Moreover, there were seven independent variables and a maximum of five demographic variables. Therefore, the maximum number of the predictors in the full 
model was 12 . According to the results of the power analysis, a sample of 122 service providers was needed for a multiple regression analysis.

\subsection{Variables and Instrumentation}

Four sections were included in the instrument. The first section asked about service to refugees, including service provision and referrals. The second section asked about the service providers' personal factors (i.e., knowledge of client [refugees], familiarity with community resources, and cultural competence). The third section gathered information on organizational culture factors (i.e., proficiency, rigidity, and resistance). The final section asked questions regarding demographic information, consisting of five personal information variables (i.e., age, biological sex, race/ethnicity, education, job title, and number of years worked in the agency) and three factors on organizational characteristics (i.e., area of service provided (state), type of agency, and refugee percentage). No one instrument could be found in the literature that had been established as appropriate for use with refugee service providers. Detailed measurement information follows (see Table 3-2).

\subsubsection{Dependent Variable}

Service provision and referrals by service providers is the dependent variable for this study. In this study, service provision is defined as the number of times service is provided for refugees. Referral is defined as researching/giving information or referring clients to relevant services. In organizational studies of business, outcomes can be measured by objective criteria, such as the number of products. Moreover, in Rizzo's 2002 study, Rizzo developed measures of social support provision by social workers to 
stroke patients based on social work initial assessment and weekly progress/final note forms. Still, to my knowledge, there are no suitable measurements for the number of service provision and referrals. Therefore, in this study, service provision and referrals were used as the dependent variables based on existing service information related to refugees.

In order to address service provision and referral, 23 questions about direct provision and referral (Stiffman et al., 2000) were created through a review of the UNHCR Resettlement Handbook of the United States of America (2014) and the addition of some services based on experiences working at a refugee center. The 23 questions regarding services and referrals can be categorized into six domains: orientation, basic needs, assistance to access benefits, employment, health, and other services. Specifically, the orientation dimension contains cultural and health safety information. Basic needs includes airport reception, financial support, case management, initial housing, essential furnishings, essential supplies, food, and clothing. The assistance to access benefits category contains enrollment in school for school-aged children, enrollment in English Language Service (ELS) class for adults, and applying for food stamps and social security cards. The employment domain consists of job training, employment, and opportunities. The health domain includes health screening, insurance, and any medical support. Lastly, other services for refugees include transportation, translation services, religious services, and legal assistance.

Respondents reported on a 7-point scale, ranging from 0 (none) to 6 (more than 51 times), to each of the service domains for which they provided service or referral in the past 12 months. Zero indicates 0 times, 1 indicates 1 to 10 times, 2 indicates 11 to 20 
times, 3 indicates 21 to 30 times, 4 indicates 31 to 40 times, 5 indicates 41 to 50 times, and 6 indicates more than 51 times. The number of services offered was summed to give a total number of services and referrals provided. A higher score indicates a higher degree of service provision and referrals. In this study, internal consistency reliability for the service provided was Cronbach's alpha .96 and service referral was .95 .

\subsubsection{Service Providers' Personal Factors}

In order to address service providers' personal factors for service provision, questions about knowledge of refugees, familiarity with community resources, and cultural competence was asked.

Service Providers' Knowledge of Refugees. Since no existing instrument to measure service providers' knowledge of refugees was found, an instrument was created to measure the service providers' awareness of the refugee population. The instrument consists of seven true/false questions. As shown in Table 3-2, below, questions came from several sources: U.S. Citizenship and Immigration Service (USCIS; n.d.), American Immigration Council (2015), the White House (2015), and the Migration Policy Institute (2015). An example of an agree/disagree question in this section is, "Refugee is someone who flees their home country because of fear for their lives or freedom." A correct answer is indicated by 1 and an incorrect answer is indicated by 0 . The possible maximum score is 7 and the minimum is 0 . A higher score indicates a higher degree of knowledge of the refugee population. 
Table 3-2 Source of Knowledge of Refugee Instrument

\begin{tabular}{l|l}
\multicolumn{1}{c|}{ Source } & \multicolumn{1}{c}{ Questionnaire } \\
\hline $\begin{array}{l}\text { U.S. Citizenship } \\
\text { and Immigration } \\
\begin{array}{l}\text { Service (USCIS; } \\
\text { n.d.) }\end{array}\end{array}$ & $\begin{array}{l}\text { Refugees are generally people outside of their country who } \\
\text { are unable or unwilling to return home because they fear } \\
\text { serious harm (True) }\end{array}$ \\
\hline $\begin{array}{l}\text { American } \\
\text { Immigration } \\
\text { Council (2015) }\end{array}$ & $\bullet \begin{array}{l}\text { Refugee, asylum seeker, and immigrant are the same meaning } \\
\text { in a broad sense (False) } \\
\text { Refugees can apply for lawful permanent resident status (also } \\
\text { as known as a green card) as soon as they are admitted to the } \\
\text { United States (False) }\end{array}$ \\
\hline $\begin{array}{l}\text { The White house } \\
\text { (2015) }\end{array}$ & $\bullet \begin{array}{l}\text { Less than 1\% of global refugee applicants are strong } \\
\text { candidates for resettlement (True) }\end{array}$ \\
\hline $\begin{array}{l}\text { Migration Policy } \\
\text { Institute (2015) }\end{array}$ & $\begin{array}{l}\text { In 2015, the three largest refugee populations in the United } \\
\text { States were from Burma, Iraq, and Somalia (True) }\end{array}$ \\
$\begin{array}{l}\text { In 2015, approximately 70,000 refugees were officially } \\
\text { resettled into the United States (True) } \\
\text { In response to safety issues, the Obama administration } \\
\text { proposed to significantly decrease the number of refugees the } \\
\text { United States accepts in following years (False) }\end{array}$ \\
\hline
\end{tabular}

Service Providers' Familiarity with Community Resources. Due to the limited research conducted on the issues of services to refugees, the researcher created an instrument to measure service providers' familiarity with community resources, based on general community resources and adapted from an existing study measuring community resources relevant to mental health (Stiffman et al., 2006). In a Stiffman and colleagues (2006) study, they created a mental health provider's resource knowledge measurement derived from 25 mental health resources. Based on their instrument creation, this study includes 20 questions related to community resources under five broad categories: financial resource, education resource, health care resource, religious and cultural resource, and professional resource. Financial resource contains information about the Temporary Assistance for Needy Families (TANF) program, SNAP (food stamp 
program), Medicaid program, food bank, and one-time cash assistance. Education resource includes information about school, ESL programs, job training, and other education resources such as parenting programs, sports, and music programs. Health care resource includes information about hospitals, community clinics, the public health organization, drug or alcohol clinics, and mental health clinics. Religious and cultural resource information includes church and ethnic organizations. Finally, professional resource includes information about social workers, counselors, psychologists, and lawyers.

The instrument consisted of 20 items and the participants reported on a 4-point scale, ranging from 1 (Not familiar at all) to 4 (Very familiar). An example of one of the statements asked is, "How familiar are you with the TANF program?" The possible maximum score is 80 and the minimum is 20 . A high cumulative score indicates a high degree of familiarity with community resources. In this study, internal consistency reliability for the overall familiarity with community resources was .92 .

Cultural Competence. The Cultural Competence Assessment Instrument (CCA) (Doorenbos, Schim, Benkert, \& Borse, 2005) was used to measure service providers' level of cultural competence since it has been demonstrated to be reliable and valid (Doorenbos, Schim, Benkert, \& Borse, 2005; Schim, Doorenbos, Miller, \& Benkert, 2003). Since the CCA was designed for a multidisciplinary setting (e.g., hospice care), items were reworded within a perspective of service providers for refugees (e.g., care reworded to service). The CCA method was generated to measure the cultural diversity experience, cultural awareness and sensitivity, and cultural competence behaviors. The CCA consists of 28 items that are divided by three subscales: a single-item index asking 
cultural diversity experience, cultural competence behaviors (CCB), and cultural awareness and sensitivity (CAS).

Participants reported the three subscales - cultural diversity experience, $\mathrm{CCB}$, and CAS - in a different way. To measure cultural diversity experience, respondents were asked to identify whether they have encountered people of various groups in the past 12 months using one question item (e.g., "How many groups have you served in the past 12 months?"). The item counted the number of groups, with a higher number indicating greater diversity experience (Doorenbos, Schim, Benkert, \& Borse, 2005). The cultural diversity experience score was not included in the calculation of the CCA score. To measure $\mathrm{CCB}$, service providers answered 16 items on a 4-point scale, ranging from 1 (Never) to 4 (Always). Service providers reported how frequently they seek information about refugees' cultural needs or conduct cultural assessments (e.g., "I use a variety of sources to learn about the cultural heritage of other people"). Lastly, CAS consisted of 11 items and included knowledge about similarities and differences in cultural beliefs and values of diverse groups (e.g., "Many aspects of culture influence service”; Starr \& Wallace, 2009). Participants reported this on a 4-point scale, ranging from 1 (Strongly disagree) to 4 (Strongly agree). The possible maximum score is 64 and the minimum is 16 for $\mathrm{CCB}$; the possible max score is 44 and the minimum is 11 for CAS. An overall score of cultural competence was obtained by summing the items, and a higher average score indicates greater cultural competence. Internal consistency reliability for the overall CCA was .92, and subscales were reported at .93 for CCB and .75 for CAS (Schim, Doorenbos, Miller, \& Benket, 2003). In this study, internal consistency reliability was reported at .87 for $\mathrm{CCB}$ and .77 for $\mathrm{CAS}$. 


\subsubsection{Organizational Culture Factors}

Organizational Culture. In order to address the organizational culture factors, the Organizational Social Context (OSC) was used. Subscales from the OSC scale (Glisson et al., 2008a) were used to assess the organizational culture of service providers' agency employers. The OSC has been confirmed in a nationally-normed agency sample (Glisson et al., 2008a) and it was originally developed to assess organizational culture and climate profiles for child welfare systems (Glisson, Green, \& Williams, 2012). However, Aarons and colleagues (2012) tested the OSC and verified the validity in a study of mental health organizations as a social service organization in a representative sample of mental health agencies from across the United States.

In this study, organizational culture measurement was used only to capture the way things are done in the organization itself (Glisson, 2007) and expectations of the service providers in their work environment (Glission \& James, 2002). Organizational climate is less concerned with what is embedded within the organization and more concerned with the impact of the shared perceptions and experiences on the individual worker (Denison, 1996; Glisson \& James, 2002). Thus, organizational climate is assessed as individual employees' perceptions of their work environment on their own functioning and well-being (James \& James, 1989).

As mentioned, organizational culture is defined as the expectations of the organization and regulations about how work is done. The measure consisted of 42 items that were divided into three domains: proficiency culture (15 items), rigidity culture (14 items), and resistance culture (13 items; Glission et al., 2008a). 
Proficiency culture characterizes service providers placing the well-being of each client first, with appropriate skills. This dimension included 2 subscales: 7 items measuring responsiveness (e.g., "Members of my organizational unit are expected to be responsive to the needs of each client") and 8 items measuring competence (e.g., "Members of my organizational unit are expected to have up-to-date knowledge"; Glisson, Williams, Green, Hemmelgarn, \& Hoagwood, 2014). A highly proficient culture is likely to be associated with better quality of service provision (Glisson et al., 2014). Internal consistency reliability for proficiency is 0.94 (Aarons et al., 2012). The possible maximum score is 60 and the minimum is 15 . A higher score indicates a higher degree of proficient organization culture. In this study, internal consistency reliability for the overall proficiency culture is .90 .

Rigidity culture implies that service providers have little discretion or flexibility in carrying out their job (Glisson, Green, \& Williams, 2012). The organization also expects that service providers follow key management decisions and bureaucratic rules and regulations (Glisson, Williams, Green, Hemmelgarn, \& Hoagwood, 2014). This dimension included 2 subscales: 7 items measuring centralization (e.g., "I have to ask a supervisor or coordinator before I do almost anything") and 7 items measuring formalization (e.g., "The same steps must be followed in processing every piece of work"; Glisson, Green, \& Williams, 2012). A highly rigid culture is more likely to be associated with poorer performance (Glisson et al., 2014). Internal consistency for alpha value of the rigidity culture is 0.81 (Aarons et al., 2012). The possible maximum score is 56 and the minimum is 14 . A higher score indicates a higher degree of rigid 
organizational culture. In this study, internal consistency reliability for the overall rigidity culture is .79.

Resistance cultures expect that service providers will have little desire of change to providing service (Glisson, Williams, Green, Hemmelgarn, \& Hoagwood, 2014). This dimension included 2 subscales: 6 items measuring apathy (e.g., "Members of my organizational unit are expected to not make waves") and 7 items measuring suppression (e.g., "Members of my organizational unit are expect to be critical"; Glisson, Green, \& Williams, 2012). A highly resistant culture is more likely to be associated with poorer performance (Glisson et al., 2014). The internal consistency reliability for resistance is 0.81 (Aarons et al., 2012). The possible maximum score is 52 and the minimum is 13 . A higher score indicates a higher degree of resistant organizational culture.

Participants reported on a 4-point scale, ranging from 1 (Not at all) to 4 (A very great extent) for all 42 questions. A higher score indicates a higher degree of resistance organizational culture. In this study, internal consistency reliability for the overall resistance culture is .62 .

\subsubsection{Demographics and Other Information}

This study included four socio-demographic and five organizational information variables due to their potential confounding effects on the outcome variable. The study measured age, sex, race/ethnicity, educational level, job title, and the number of years the participants have worked in the agency. In addition, to measure information about their organization, participants were asked which state their organization serves, type of agency, and the percentage of refugee clients in their total number of clients.

Age. This item was defined as a continuous variable. 
Biological Sex. It consisted of two categories: female and male.

Race/Ethnicity. It consisted of six categories: White, Hispanic or Latino, Black or African American, Native American or American Indian, Asian/Pacific Islander, and other.

Education Level. It consisted of five categories: less than high school, high school (GED), undergraduate degree, graduate degree, and other.

Job Title. Job title is a set of job roles across organizations that have common work requirements such as tasks, responsibilities, goals, or methods of achieving these responsibilities (Morgeson, Dierdorff, \& Hmurovic, 2010). Therefore, different job positions have different responsibilities that could affect service provision outcomes. This item was defined as a continuous variable.

Number of Years Worked in the Agency. Years of work experience at an agency impacts the service provider's familiarity with clients, resources, and the organization. Therefore, different work experience could result in a different amount of service provision. This item was defined as a continuous variable.

State Where Organization Serves. Even though there is no study examining the impact of state on service provision to refugees, it is possible that different states could have different service outcomes because of regional resources. Since this study included surveys across the US, it may be necessary to know the organization's location. This item was defined as a continuous variable.

Type of Agency. Even though there is no study examining the impact of the type of agency on service provision to refugees, it is possible that different agencies could have different service outcomes simply because of the agency type. This is because some 
agencies might provide services only for refugees but others might not. Moreover, there might be many types of agencies to provide service to refugees; therefore, the survey measured the number of various organization types that provide service to refugees. This item was defined as a continuous variable.

Percentage of Refugee Clients. Service providers who work with refugees were the participants of this study. However, providers may have varying levels of refugee clients based on the type of organization and the number of refugees the organization serves. Service providers who have more refugee clients may have more information about refugees than those who serve fewer refugee clients. This item was defined as a continuous variable.

Table 3-3 Overview of Instruments

\begin{tabular}{|c|c|c|c|c|}
\hline & & Variables & Sources & Reliability \\
\hline \multirow{12}{*}{$\begin{array}{l}\text { Dependent } \\
\text { Variable }\end{array}$} & \multirow{6}{*}{$\begin{array}{l}\text { Service } \\
\text { provision } \\
\text { for } \\
\text { refugee }\end{array}$} & 1.Orientation & \multirow{6}{*}{ (UNHCR, 2014) } & \multirow{6}{*}{$\begin{array}{l}\text { Cronbach's } \\
\text { alpha }=.96\end{array}$} \\
\hline & & 2.Basic needs & & \\
\hline & & $\begin{array}{l}\text { 3.Assistance to access } \\
\text { benefits }\end{array}$ & & \\
\hline & & 4.Employment & & \\
\hline & & 5.Health & & \\
\hline & & 6.Other services & & \\
\hline & \multirow{6}{*}{$\begin{array}{l}\text { Referrals } \\
\text { for } \\
\text { refugee }\end{array}$} & 1.Orientation & \multirow{6}{*}{ (UNHCR, 2014) } & \multirow{6}{*}{$\begin{array}{c}\text { Cronbach's } \\
\text { alpha }=.95\end{array}$} \\
\hline & & 2.Basic needs & & \\
\hline & & $\begin{array}{l}\text { 3.Assistance to access } \\
\text { benefits }\end{array}$ & & \\
\hline & & 4.Employment & & \\
\hline & & 5.Health & & \\
\hline & & 6.Other services & & \\
\hline \multirow{6}{*}{$\begin{array}{c}\text { Independent } \\
\text { Variables }\end{array}$} & \multicolumn{4}{|c|}{ Personal Factors } \\
\hline & \multirow{2}{*}{\multicolumn{2}{|c|}{$\begin{array}{l}\text { Knowledge of refugee } \\
\text { Familiarity with community } \\
\text { resources }\end{array}$}} & & $\mathrm{n} / \mathrm{a}$ \\
\hline & & & & $\begin{array}{l}\text { Cronbach's } \\
\text { alpha }=.92\end{array}$ \\
\hline & \multicolumn{2}{|c|}{ Cultural competence behavior } & \multirow{2}{*}{$\begin{array}{c}\text { (Doorenbos et al., } \\
\text { 2005) }\end{array}$} & $\begin{array}{l}\text { Cronbach's } \\
\text { alpha }=.87\end{array}$ \\
\hline & \multicolumn{2}{|c|}{ Cultural awareness/sensitivity } & & $\begin{array}{l}\text { Cronbach's } \\
\text { alpha }=.77\end{array}$ \\
\hline & \multicolumn{4}{|c|}{ Organizational Culture Factors } \\
\hline
\end{tabular}




\begin{tabular}{|c|c|c|c|}
\hline & Culture: Proficiency & \multirow{3}{*}{$\begin{array}{c}\text { (Glisson et al., } \\
\text { 2008) }\end{array}$} & $\begin{array}{c}\text { Cronbach's } \\
\text { alpha }=.90\end{array}$ \\
\hline & Culture: Rigidity & & $\begin{array}{l}\text { Cronbach's } \\
\text { alpha }=.79\end{array}$ \\
\hline & Culture: Resistance & & $\begin{array}{l}\text { Cronbach's } \\
\text { alpha }=.62\end{array}$ \\
\hline \multirow{5}{*}{ Demographics } & Age & & \\
\hline & Biological Sex & & \\
\hline & Race/Ethnicity & & \\
\hline & Educational level & & \\
\hline & \begin{tabular}{|l} 
Job title \\
\end{tabular} & & \\
\hline
\end{tabular}

\subsection{Data Analysis Strategies}

A series of quantitative tests were utilized to answer the research questions. The statistical analysis was divided into four components: descriptive analysis, univariate analysis, bivariate analysis, and multiple regression (See Table 3-4).

Before analysis of the main hypotheses, a preliminary analysis was conducted. A univariate analysis was conducted to calculate mean, range, and standard deviations of all variables.

The first research question was answered by conducting bivariate analyses of the characteristics of the independent variables and the dependent variables. Moreover, it tested differences in service provision and referrals by demographics and independent variables.

To address research questions 2-1 and 2-2, before the multiple regression analysis, correlation analysis was used to test the relationship among all study variables. Then, multiple regression was completed to determine the strength of the relationship between the predictor variables and the number of service provision. Multiple regression models were used to examine the relative effects of multiple independent variables on service provision and referrals. 
Table 3-4 Data Analysis Summary

\begin{tabular}{l} 
Research Question \\
\hline Preliminary Analysis \\
\hline \\
1. What type of services are currently being \\
provided to meet the needs of refugees?
\end{tabular}
provided to meet the needs of refugees?
- Univariate analysis

- Bivariate analysis conducted. How personal factors, organizational culture factors, and dependent variables (service provision and referrals) differ by demographic variables
2-1. What are the relative effects of personal factors (knowledge of refugees, familiarity with community resources, and cultural competence) and organizational culture factors (proficiency, rigidity, and resistance) on service provision for refugees? (direct association)
- Correlations conducted for dependent variables and independent variables in order to examine associations

- Regression analysis conducted. How much service provision is accounted for personal factors and organizational culture factors and how strongly they related to service provision
2-2. What are the relative effects of personal factors (knowledge of refugees, familiarity with community resources, and cultural competence) and organizational culture factors (proficiency, rigidity, and resistance) on service referrals for refugees? (direct association)
- Correlations conducted for dependent variables and independent variables in order to examine associations

- Regression analysis conducted. How much service provision is accounted for personal factors and organizational culture factors and how strongly they related to service referral
3. Does cultural competence behavior and cultural awareness/sensitivity mediate the associations between familiarity with community resources and service provision or referrals for refugees? (indirect association)
- Mediation analysis conducted. Estimation of path-coefficients and test the significance of indirect effects

- Using PROCESS SPSS Macro (Preacher \& Hayes's, 2008)
4. Does cultural competence behavior and cultural awareness/sensitivity mediate the associations between work experience and service provision or referrals for refugees? (indirect association)
- Mediation analysis conducted. Estimation of path-coefficients and test the significance of indirect effects

- Using PROCESS SPSS Macro (Preacher \& Hayes's, 2008) 
Lastly, to address research questions 3 and 4, mediation analysis was conducted. Mediation is to understand how changes are transmitted from causal variables through intervening variables, which in turn leads to changes in an outcome (Little, 2013). In this study, mediation models were used to examine mediators' effect on the association between multiple independent variables and service provision and referrals.

To examine mediation effect in this study, the researchers employed Preacher and Hayes's (2008) PROCESS SPSS Macro using bootstrapping to obtain estimates of pathcoefficients and test the significance of indirect effects (mediation effects). Below are reasons why this research utilized Preacher and Hayes's PROCESS.

Baron and Kenny's (1986) "causal steps" method is the most well-known mediation analysis. The causal steps assume that certain steps must be met for mediation to occur. For example, first, there should be a significant correlation between the independent variable $(\mathrm{X})$ and the dependent variables $(\mathrm{Y})$; if the first step is not met, the process could not move on to the next step. Second, there should be a significant correlation between $\mathrm{X}$ and the mediator variable (M). The third and fourth steps are that there should be a significant correlation between $\mathrm{M}$ and $\mathrm{Y}$, and when controlled for $\mathrm{M}$, the correlation should no longer be statistically significant (complete mediation) or have a significant reduction of the direct effect (partial mediation; Bailey, n.d; Hayes, 2009; Stage, Carter, \& Nora, 2004). However, the causal steps approach has been criticized by many researchers. They are especially concerned with the concept that the path of X -> Y (c path in Figure 3-6) has to be significant in order to detect mediation effect (Hayes, 2009; Hayes, 2013; Rucker, Preacher, Tormala, \& Petty, 2011; Shrout \& Bolger, 2002). Additionally, for this concept, they felt it failed to test the hypotheses. However, the 
indirect effect ( $\mathrm{a}^{*} \mathrm{~b}$ path) might be detected even when the $\mathrm{c}$ effect is not significant (Rucker, Preacher, Tormala, \& Petty, 2011). This is because X might have a stronger relationship with M (path a) than with $\mathrm{Y}$ (c' path), and it could lead to a stronger indirect effect ( $a * b$ path) than total effect (c path). Thus, the $a * b$ path can be significant even when the $\mathrm{c}$ path is not. Indeed, focusing on the significance of the $\mathrm{X}->\mathrm{Y}$ relationship might be unnecessary (Bailey, n.d).

The other reason for utilizing the PROCESS approach using bootstrapping, which is beneficial for a small sample size, is that it is used for testing the mediation effect (Preacher \& Hayes, 2008). Bootstrapping is a method of repeatedly sampling from the data set and estimating the indirect effect in each resampled data set. By repeating this process, the sampling distribution of $a * b$ was created and used to construct confidence intervals for the indirect effect. Moreover, bootstrap samples to derive the $95 \%$ biascorrected confidence intervals (CI) for the indirect effects. CI (95\%) not including zero indicate significant indirect effects and, thus, that the effect of the independent variable on the dependent variable is mediated by the mediators (Hayes, 2013). Therefore, this study used PROCESS to examine cultural competence mediation effects.

Figure 3-6 displays a multiple mediator model. It illustrates the effects (represented as unstandardized coefficients) of the independent variable on the proposed mediator variable (a paths), the effects of the mediator variables on the dependent variable taking the other mediators into account (b path), the direct effect (c' path), and the specific indirect effects ( $a * b$ paths). 

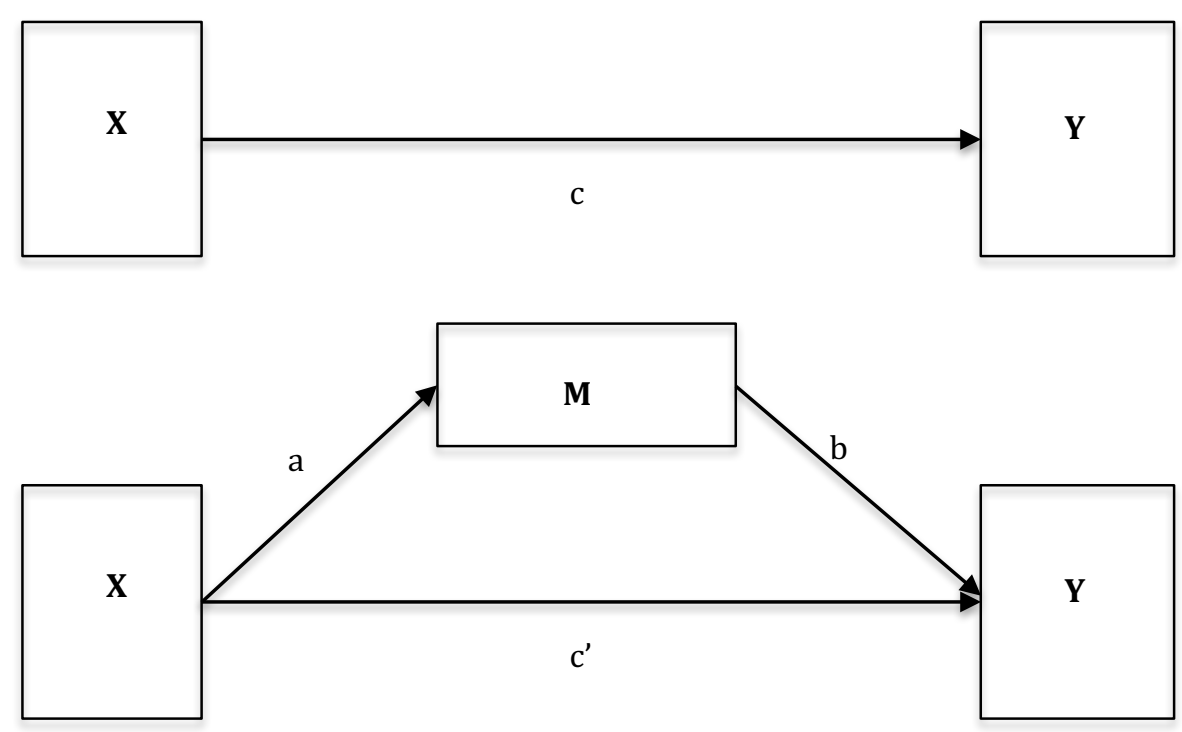

Figure 3-6 Mediation Model

\subsection{Summary}

Chapter 3 presented the research design, participant sampling, sample size, instruments, human subjects protection, and data analysis to identify the personal and organizational culture factors of service providers who provide service provision and referrals for refugees. An online self-administered questionnaire was used to collect data that were used to measure major study variables: service provision, referrals, personal factors, organizational culture factors, and demographics. Data analyses were described in detail. The following chapter presents the results. 


\section{CHAPTER 4: RESULTS}

This chapter describes the sample of refugee service providers who participated in the survey and provides the data analysis results intended to answer the five main research questions. The aims of the study's data analysis were: 1) to gain an understanding of the characteristics of refugee service providers, 2) to examine relative effects of personal and organizational culture factors on service provision and referrals for refugees, and 3) to explore the mediation effect of cultural competence. In order to answer the research questions and test the hypotheses, this study developed a data analysis plan and analyzed the data based on the plan.

This chapter discusses the survey procedure and data analysis preparation, including handling missing data, summed subscale, normality and reliability, followed by socio-demographic characteristics of the sample, descriptive findings of the study variables, bivariate correlation among the main variables, and explanation of service provision. Lastly, the results of testing the research hypotheses by utilizing multiple regression analysis and mediation analysis are presented.

\subsection{Survey Procedure and Sample Size}

The online survey was started in February and closed in August. Approximately 735 refugee service providers were selected and emailed the research study questionnaire (Appendix A). Three follow-up email communications were sent at 1-week intervals encouraging practitioners to participate in the research by completing the online questionnaire. Approximately $24 \%$ of practitioners completed the questionnaire $(\mathrm{n}=$ 170). The collected data in Qualtrics were automatically exported into the IBM Statistical 
Package for Social Science (SPSS, Version 23) software program. A non-response unit was created for missing data, and the researcher excluded those cases in the final sample. Finally, 146 cases were used for the descriptive analysis and 115 cases were used for the regression analysis.

\subsection{Data Analysis Preparation}

Four steps were used to prepare for data analysis: (1) handling missing data, (2) summed subscale, (3) normality check, (4) reliability and multicollinearity check. In order to use multiple regression and mediation analysis, it was necessary to eliminate all cases with missing data for one or more variables. While missing data were expected to be minimal, it is important to be able to utilize whatever data were provided, rather than excluding participants due to one or more answers not being given. Therefore, an Expectation-maximization (EM) algorithm was used in the study. An EM algorithm is a general method for obtaining the maximum likelihood estimation within statistical analysis with incomplete data (Allison, 2002; Dempster et al., 1977; Ding \& Song, 2016). The EM algorithm steps are: “(1) replace missing values by estimated values, (2) estimate parameters, (3) reestimate the missing values assuming the new parameter estimates are correct, (4) reestimate parameters and so forth, iterating until convergence" (Little \& Rubin, 1987, p. 129). Using an EM algorithm technique in SPSS, missing values were filled out for each possible completion of the missing values rather than picking the single most likely completion of the missing values in each iteration (Do \& Batzoglou, 2008). 
Next, subscale scores were summed and the overall total score variables were computed by adding the summed subscale score to create totaled scale variables. The variables were service provision, service referral, knowledge of refugee, familiarity with community resource, cultural competence (cultural competence behavior and cultural awareness/sensitivity), and organizational culture (proficiency, rigidity, and resistance).

Third, the variables were screened to check for data normality. Normality of residuals is important for showing that this study did not violate the basic assumption. According to Habeck and Brickman (2014), if the residuals in a linear regression are well behaved, the distribution of variables is irrelevant. The standardized residual histogram and normal P-P plot of regression standardized residual supported assessment of skewness. No violations of normality were found (see detailed information about normality in Appendix E).

Lastly, Cronbach's alphas of the scale variables were checked for internal consistency. Moreover, the tolerance levels and the variance inflation factors (VIF) levels were checked to ensure that the explanatory variables were not linearly related. Otherwise, the problem of multicollinearity can occur in the model, which is a potential problem in all regression analysis and mediation relationships (Kline, 2015; Sinan \& Alkan, 2015; Thompson, Kim, Aloe, \& Becker, 2017). Particularly, this data could possibly have a nested data effect between personal factors and organizational factors, which means that all predictors are highly interrelated in the model. Therefore, ensuring that there was no multicollinearity among variables was an important step before conducted a regression analysis. Generally, tolerance should be at least 0.1 and VIF values should be less than 10 (Tabachnick \& Fidell, 2007). By checking tolerance and 
VIF values, multicollinearity is not likely to be an issue. Detailed information about reliability is in the descriptive findings of the study variables.

\subsection{Descriptive Statistics}

The descriptive outline of the study sample includes demographic characteristics, agency characteristics, and main variables. The final sample consisted of 146 responses from service providers.

\subsubsection{Demographics}

Table 4-1 presents the demographic characteristics of the study sample. The average age of participants was 36.3 years old $(S D=10.5)$ with a range between 23 and 75 years. The majority of the study participants reported their race/ethnicity to be white $(75.3 \%)$ and the sample was predominately female $(81.5 \%)$. Over half of the study participants completed a graduate degree and higher (59.6\%). The vast majority of participants $(86.3 \%)$ have not been a refugee. Further, $62.3 \%$ of study participants were employed in a refugee resettlement related job.

Table 4-1 Socio-demographic Characteristics of Study Participants $(\mathrm{N}=146)$

\begin{tabular}{|c|c|c|c|c|}
\hline & $\%$ & $\mathrm{n}$ & $\mathrm{M}$ & $\mathrm{SD}$ \\
\hline \multicolumn{5}{|l|}{ Age } \\
\hline $20-29$ & 32.9 & 48 & & \\
\hline $30-39$ & 42.5 & 62 & & \\
\hline $40-49$ & 11.6 & 17 & & \\
\hline $50-59$ & 7.5 & 11 & & \\
\hline 60 and more & 5.5 & 8 & & \\
\hline Age in years (range $23-75$ ) & & & 36.3 & 10.5 \\
\hline Gender & & & & \\
\hline
\end{tabular}




\begin{tabular}{lcc}
\hline Male & 18.5 & 27 \\
Female & 81.5 & 119 \\
Race/Ethnicity & & \\
White & 75.3 & 110 \\
Hispanic or Latino & 5.5 & 8 \\
Asian & 8.7 & 13 \\
Black or African American & 6.2 & 9 \\
Other & 4.1 & 7 \\
Education & & \\
Undergraduate degree and lower & 40.4 & 59 \\
Graduate degree and higher & 59.6 & 87 \\
Was a Refugee & & \\
Yes & 13.7 & 20 \\
No & 86.3 & 126 \\
Job Title & & \\
Resettlement related & 62.3 & 91 \\
Employment related & 17.1 & 25 \\
Education related & 11.0 & 16 \\
Healthcare related & 9.6 & 14 \\
\hline
\end{tabular}

\subsubsection{Characteristics of Agency}

Table 4-2 shows information about the agencies where the study participants work. Most of the respondents work in the Midwest (35.6\%) and South (36.3\%) regions. The vast majority of participants (84.9\%) are working at a refugee resettlement agency. The average number of employees of participants' agencies was $39.9(S D=27.9)$ with a range of 1 to 100 . Further, on average, the percentage of refugee clients served by the agency during the last 12 months was $79.3(S D=27.2)$ with a range of 10 to 100 .

Table 4-2 Characteristics of Agency where Study Participants work (N=146)

\begin{tabular}{|c|c|c|c|c|}
\hline Variables & $\%$ & $\mathbf{n}$ & $\mathbf{M}$ & SD \\
\hline State & & & & \\
\hline
\end{tabular}




\begin{tabular}{llllr}
\hline Midwest & 35.6 & 52 & & \\
South & 36.3 & 53 & & \\
West & 9.6 & 14 & & \\
$\quad$ Northeast & 18.5 & 27 & & \\
Type of agency & & & & \\
$\quad$ Refugee Resettlement Agency & 84.9 & 124 & & \\
$\quad \begin{array}{l}\text { Social Service Agency } \\
\text { Religious Agency }\end{array}$ & 13.7 & 20 & & \\
\cline { 2 - 5 } & 1.4 & 2 & & \\
\hline Number of employees (1-100) & & & M & SD \\
$\begin{array}{l}\text { Percentage of refugee client } \\
\text { (range 10-100) }\end{array}$ & & & 39.9 & 27.9 \\
\hline
\end{tabular}

\subsubsection{Characteristics of Main Variables}

Table 4-3 presents a summary of descriptive findings of the main variables and the computed study variable. The summed study variables included service provision (service provision and service referral), knowledge of refugee, familiarity with community resources, cultural competence (cultural competence behavior and cultural awareness and sensitivity), and organizational culture (proficiency, rigidity, and resistance). In addition, internal reliability of each scale was examined and is presented in Table 4-3.

Table 4-3 Range, Mean, and SD among Main Scale Variables (N=115)

\begin{tabular}{lcccc}
\hline & Range & Mean & SD & Alpha \\
\cline { 2 - 5 } Service Provision & $0-138$ & 63.6 & 43.5 & .96 \\
$\quad$ Service Provision & $0-138$ & 35.5 & 35.8 & .95 \\
Service Referral & & & & \\
Personal Factors & $3-7$ & 5.2 & .9 & $\mathrm{n} / \mathrm{a}$ \\
$\quad$ Knowledge of Refugee & $36-80$ & 64.1 & 10.5 & .92 \\
Familiarity with Community Resource & & & &
\end{tabular}




\begin{tabular}{ccccc}
\cline { 1 - 2 } Cultural Competence: & & & & \\
Cultural Competence Behavior (CCB) & $29-64$ & 55.3 & 7.0 & .87 \\
Cultural Awareness and Sensitivity (CAS) & $6-44$ & 37.0 & 4.5 & .78 \\
Organizational Factors & & & & \\
Proficiency & $23-60$ & 48.7 & 7.1 & .90 \\
Rigidity & $18-47$ & 29.0 & 6.0 & .79 \\
Resistance & $19-43$ & 26.0 & 4.5 & .62 \\
\hline
\end{tabular}

Service Provision. There were two types of scales: number of service provision and number of service referrals. It was created through a review of the UNHCR Resettlement Handbook of the United States of America (2014) and the addition of some services based on experiences working at a refugee center. There are six subdomains for each scale: orientation, basic needs, assistance to access benefits, employment, health, and other services. The exact number of service provision was not measured. Instead, there were six numbers, each number indicated the range of number of provision. A higher number indicates that services were provided and referred more. A detailed explanation is provided in Chapter 3 .

The mean score of service provision was $63.6(S D=43.5)$, and the scores ranged from 0 to 138 . The Cronbach's alpha was .96 , which indicates a high level of reliability. Next, service referrals ranged from 0 to 138 , with a mean of $35.5(S D=35.8)$. The theoretical range of this scale was from 0 to 138 . The Cronbach's alpha was .95 , which indicates a high level of reliability.

As shown in Table 4-4, service provision by domains, the mean score of service provided for Domain 1 (orientation) was $4.2(S D=1.6)$, and the score ranged from 2 to 6 . The mean score of service provided for Domain 2 (basic needs) was $16.4(S D=5.4)$, and the score ranged from 8 to 24 . In addition, the mean score of service provided for Domain 
3 (assistance to access benefits) was $7.8(S D=2.9)$, and the score ranged from 4 to 12 . For service provided for Domain 4 (employment), the mean score was $4.1(S D=1.6)$ and the score ranged from 2 to 6 . The mean score of service provided for Domain 5 (health) was $5.6(S D=2.2)$, and the score ranged from 3 to 9 . Lastly, the mean score of service provided for Domain 6 (other services) was $7.4(S D=2.1)$, and the score ranged from 4 to 12.

Next, for service referral subdomains, the mean score of Domain 1 (orientation) was $2.8(S D=1.3)$, and the score ranged from 2 to 6 . For service referral Domain 2 (basic needs), the mean score was $12.5(S D=4.5)$, and the score ranged from 8 to 24 . Moreover, the mean score for service referral Domain 3 (assistance to access benefits) was 6.6 $(S D=2.5)$, and the score ranged from 4 to 12 . For service referral Domain 4 (employment), the mean score was $3.3(S D=1.5)$, and the score ranged from 2 to 6 . In addition, the mean score of service referral Domain 5 (health) was $5.2(S D=2.1)$, and the score ranged from 4 to 12. Lastly, the mean score of service referral Domain 6 (other services) was $6.3(S D=2.1)$, and the score ranged from 4 to 12 . Domain 2, basic needs, was the most frequently provided and referred to refugees. In contrast, orientation (Domain 1) service was the least provided and referred among the six domains.

Table 4-4 Range, Mean, and SD among Dependent Variable by Domains ( $\mathrm{N}=115)$

\begin{tabular}{llll}
\hline & \multicolumn{1}{c}{ Range } & Mean & SD \\
\cline { 2 - 4 } Service Provision & & & \\
Domain 1: Orientation & $2-6$ & 4.2 & 1.6 \\
Domain 2: Basic Needs & $8-24$ & 16.4 & 5.4 \\
Domain 3: Assistance to Access to Benefits & $4-12$ & 7.8 & 2.9 \\
Domain 4: Employment & $2-6$ & 4.1 & 1.6 \\
Domain 5: Health & $3-9$ & 5.6 & 2.2 \\
\hline
\end{tabular}




\begin{tabular}{llll}
\hline $\begin{array}{l}\text { Domain 6: Other Services } \\
\text { Service Referral }\end{array}$ & $4-12$ & 7.4 & 2.1 \\
Domain 1: Orientation & $2-6$ & 2.8 & 1.3 \\
Domain 2: Basic Needs & $8-24$ & 12.5 & 4.5 \\
Domain 3: Assistance to Access to Benefits & $4-12$ & 6.6 & 2.5 \\
Domain 4: Employment & $2-6$ & 3.3 & 1.5 \\
Domain 5: Health & $3-9$ & 5.2 & 2.1 \\
Domain 6: Other Services & $4-12$ & 6.3 & 2.1 \\
\hline
\end{tabular}

Tables 4-5 and 4-6 indicate detailed information of service provision and referrals of refugee service providers by type of services. The most provided service by refugee service providers was case management followed by transportation service. In addition, the most referred service by service providers was immigration legal service referral followed by health screening and health insurance service referral. 
Table 4-5 Service Provision by Type of Services $(\mathrm{N}=115)$

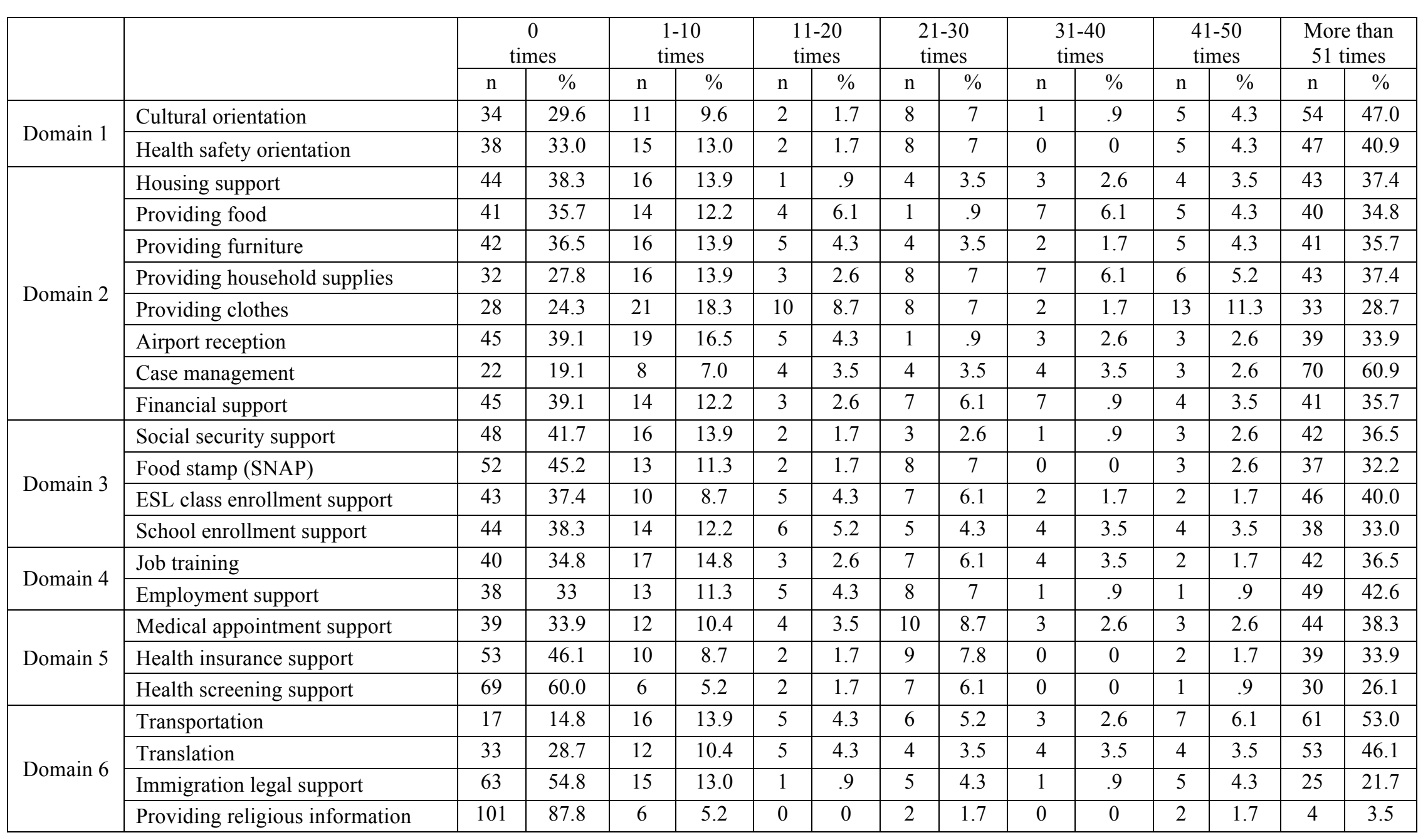


Table 4-6 Service Referral by Type of Services $(\mathrm{N}=115)$

\begin{tabular}{|c|c|c|c|c|c|c|c|c|c|c|c|c|c|c|c|}
\hline & & \multicolumn{2}{|c|}{$\begin{array}{c}0 \\
\text { times }\end{array}$} & \multicolumn{2}{|c|}{$\begin{array}{c}1-10 \\
\text { times }\end{array}$} & \multicolumn{2}{|c|}{$\begin{array}{l}11-20 \\
\text { times }\end{array}$} & \multicolumn{2}{|c|}{$\begin{array}{l}21-30 \\
\text { times }\end{array}$} & \multicolumn{2}{|c|}{$\begin{array}{l}31-40 \\
\text { times }\end{array}$} & \multicolumn{2}{|c|}{$\begin{array}{l}41-50 \\
\text { times }\end{array}$} & \multicolumn{2}{|c|}{$\begin{array}{l}\text { More than } \\
51 \text { times }\end{array}$} \\
\hline & & $\mathrm{n}$ & $\%$ & $\mathrm{n}$ & $\%$ & $\mathrm{n}$ & $\%$ & $\mathrm{n}$ & $\%$ & $\mathrm{n}$ & $\%$ & $\mathrm{n}$ & $\%$ & $\mathrm{n}$ & $\%$ \\
\hline \multirow{2}{*}{ Domain 1} & Cultural orientation & 83 & 72.2 & 8 & 7.0 & 2 & 1.7 & 4 & 3.5 & 1 & .9 & 1 & .9 & 16 & 13.9 \\
\hline & Health safety orientation & 85 & 73.9 & 8 & 7.0 & 5 & 4.3 & 2 & 1.7 & 0 & 0 & 0 & 0 & 15 & 13.0 \\
\hline \multirow{8}{*}{ Domain 2} & Housing support & 58 & 50.4 & 30 & 26.1 & 10 & 8.7 & 4 & 8.7 & 0 & 0 & 2 & 1.7 & 11 & 9.6 \\
\hline & Providing food & 56 & 48.7 & 20 & 17.4 & 6 & 5.2 & 4 & 3.5 & 2 & 1.7 & 5 & 4.3 & 22 & 19.1 \\
\hline & Providing furniture & 70 & 60.9 & 16 & 13.9 & 7 & 6.1 & 5 & 4.3 & 1 & .9 & 1 & .9 & 15 & 13.0 \\
\hline & Providing household supplies & 73 & 63.5 & 18 & 15.7 & 4 & 3.5 & 5 & 4.3 & 2 & 1.7 & 0 & 0 & 13 & 11.3 \\
\hline & Providing clothes & 61 & 53.0 & 15 & 13.0 & 5 & 4.3 & 6 & 5.2 & 5 & 4.3 & 3 & 2.6 & 20 & 17.4 \\
\hline & Airport reception & 98 & 85.2 & 6 & 5.2 & 1 & .9 & 1 & .9 & 0 & 0 & 0 & 0 & 9 & 7.8 \\
\hline & Case management & 66 & 57.4 & 10 & 8.7 & 8 & 7.0 & 7 & 6.1 & 2 & 1.7 & 1 & .9 & 21 & 18.3 \\
\hline & Financial support & 59 & 51.3 & 14 & 12.2 & 6 & 5.2 & 8 & 7.0 & 2 & 1.7 & 3 & 2.6 & 23 & 20.0 \\
\hline \multirow{4}{*}{ Domain 3} & Social security support & 72 & 62.6 & 19 & 16.5 & 3 & 2.6 & 1 & .9 & 1 & .9 & 0 & 0 & 19 & 16.5 \\
\hline & Food stamp (SNAP) & 60 & 52.2 & 15 & 13.0 & 4 & 3.5 & 8 & 7.0 & 1 & .9 & 2 & 1.7 & 25 & 21.7 \\
\hline & ESL class enrollment support & 55 & 47.8 & 15 & 13.0 & 7 & 6.1 & 7 & 6.1 & 3 & 2.6 & 2 & 1.7 & 26 & 22.6 \\
\hline & School enrollment support & 64 & 55.7 & 18 & 15.7 & 5 & 4.3 & 3 & 2.6 & 2 & 1.7 & 3 & 2.6 & 20 & 17.4 \\
\hline \multirow{2}{*}{ Domain 4} & Job training & 64 & 55.7 & 11 & 9.6 & 9 & 7.8 & 5 & 4.3 & 4 & 3.5 & 4 & 3.5 & 18 & 15.7 \\
\hline & Employment support & 55 & 47.8 & 15 & 13.0 & 5 & 4.3 & 9 & 7.8 & 4 & 3.5 & 3 & 2.6 & 24 & 20.9 \\
\hline \multirow{3}{*}{ Domain 5} & Medical appointment support & 57 & 49.6 & 10 & 8.7 & 9 & 7.8 & 8 & 7 & 3 & 2.6 & 3 & 2.6 & 25 & 21.7 \\
\hline & Health insurance support & 55 & 47.8 & 14 & 12.2 & 7 & 6.1 & 5 & 4.3 & 2 & 1.7 & 5 & 4.3 & 27 & 23.5 \\
\hline & Health screening support & 61 & 53.0 & 12 & 10.4 & 2 & 1.7 & 7 & 6.1 & 0 & 0 & 3 & 2.6 & 30 & 26.1 \\
\hline \multirow{4}{*}{ Domain 6} & Transportation & 67 & 58.3 & 15 & 13.0 & 4 & 3.5 & 4 & 3.5 & 1 & .9 & 3 & 2.6 & 21 & 18.3 \\
\hline & Translation & 69 & 60.0 & 10 & 8.7 & 7 & 6.1 & 9 & 7.8 & 2 & 1.7 & 2 & 1.7 & 16 & 13.9 \\
\hline & Immigration legal support & 50 & 43.5 & 19 & 16.5 & 7 & 6.1 & 10 & 8.7 & 6 & 5.2 & 4 & 3.5 & 19 & 16.5 \\
\hline & Providing religious information & 77 & 67.0 & 18 & 15.7 & 4 & 3.5 & 2 & 1.7 & 0 & 0 & 2 & 1.7 & 12 & 10.4 \\
\hline
\end{tabular}


Knowledge of Refugee. The mean score of summed knowledge of refugee was $5.2(S D=.9)$ and the score ranged from 3 to 7 . The theoretical score range is from 0 to 7 . This scale is a correct-false test, not a Likert scale. Therefore, it did not have the Cronbach's Alpha coefficient value (Kisa, 2015). Most of service providers (99.1\%) knew the definition of refugee. On the other hand, only 33 service providers out of 115 had a wrong answer about refugee admission under the Obama administration. Detailed information of knowledge of refugee by type of question is shown in Table 4-7.

Table 4-7 Knowledge of Refugee by Type of Question (N=115)

\begin{tabular}{lcc}
\hline & \multicolumn{2}{c}{$\begin{array}{c}\text { Frequency and Percent of } \\
\text { Correct Answer }\end{array}$} \\
\cline { 2 - 3 } & $\mathrm{n}$ & $\%$ \\
\cline { 2 - 3 } $\begin{array}{l}\text { Refugees are generally people outside of their country who are } \\
\text { unable or unwilling to return home because of they fear } \\
\text { serious harm (True) }\end{array}$ & 114 & 99.1 \\
$\begin{array}{l}\text { Refugee, asylum seeker, and immigrant are the same meaning } \\
\text { in a broad sense (False) }\end{array}$ & 92 & 80.0 \\
$\begin{array}{l}\text { Less than } 1 \% \text { of global refugee population applicants who are } \\
\text { strong candidates for resettlement (True) }\end{array}$ & 65 & 56.5 \\
$\begin{array}{l}\text { In } 2015, \text { the three largest refugee population in the United } \\
\text { States were Burma, Iraq, and Somalia(True) }\end{array}$ & 94 & 81.7 \\
$\begin{array}{l}\text { In } 2015, \text { around } 70,000 \text { refugees were officially resettled into } \\
\text { the United States (True) }\end{array}$ & 107 & 93.0 \\
$\begin{array}{l}\text { In response to safety issue, the Obama administration proposed } \\
\text { to significantly decrease the number of refugees the United }\end{array}$ & 38 & 33.0 \\
$\begin{array}{l}\text { States accepts following years (False) } \\
\text { Refugee can apply for lawful permanent resident status (also } \\
\text { as known as a green card) as soon as being admitted to the } \\
\text { United States (False) }\end{array}$ & 88 & 76.5 \\
\hline
\end{tabular}


Familiarity with Community Resource. For the summed familiarity with community resources, the mean score was $64.1(S D=10.5)$ and the score ranged from 36 to 80 . The theoretical score range is from 20 to 80 . The scale was considerably reliable with a Cronbach's Alpha coefficient of .92. The most familiar community resource for refugee service providers was the ESL program (94.8\%) followed by the Medicaid program $(92.2 \%)$ and social worker $(91.3 \%)$. On the other hand, the community resource study participants were least familiar with was the drug or alcohol clinic (57.4\%). Table 4-8 presents the detailed information of service providers' responses.

Table 4-8 Familiarity with Community Resources by Type of Question $(\mathrm{N}=115)$

\begin{tabular}{lcccc}
\hline & \multicolumn{2}{c}{ Frequency and Percent of Familiarity } \\
\cline { 2 - 5 } & \multicolumn{2}{c}{ Familiar } & \multicolumn{2}{c}{ Not Familiar } \\
\cline { 2 - 5 } & $\mathrm{n}$ & $\%$ & $\mathrm{n}$ & $\%$ \\
\cline { 2 - 5 } TANF program & 90 & 78.2 & 25 & 21.8 \\
\hline Food stamp program & 107 & 93.0 & 8 & 7.0 \\
Medicaid program & 106 & 92.2 & 9 & 7.8 \\
Food bank & 94 & 81.7 & 21 & 18.3 \\
One-time cash assistance & 80 & 69.6 & 35 & 30.4 \\
Schools & 103 & 89.6 & 12 & 10.4 \\
ESL program & 109 & 94.8 & 6 & 5.2 \\
\hline Job training & 97 & 84.3 & 18 & 15.7 \\
Other education program & 78 & 67.8 & 37 & 32.2 \\
Hospital & 101 & 87.8 & 14 & 12.2 \\
Community clinic & 97 & 84.3 & 18 & 15.7 \\
Public health organization & 86 & 74.8 & 29 & 25.2 \\
Drug or alcohol clinic & 49 & 42.6 & 66 & 57.4 \\
Mental health clinic & 81 & 70.4 & 34 & 29.6 \\
Church & 76 & 66.1 & 39 & 33.9 \\
\hline Ethnic organization & 84 & 73.0 & 31 & 27.0 \\
Social worker & 105 & 91.3 & 10 & 8.7 \\
Counselor & 98 & 85.2 & 17 & 14.8 \\
\hline Psychologist & 86 & 74.8 & 29 & 25.2 \\
\hline Lawyer & 86 & 74.8 & 29 & 25.2 \\
\hline & & & & \\
\hline
\end{tabular}


Cultural Competence. There were two scales that measured cultural competence of refugee service providers. First, for the summed cultural competence behavior (CCB), the mean score was $55.3(S D=7.0)$ and the score ranged from 29 to 64 . The theoretical score range was from 16 to 64 . The Cronbach's alpha coefficient was .87, which indicates a reliable scale with this sample.

Detailed cultural competence behavior by type of question is included in Table 49. Service providers who have higher cultural competence behavior, reported similar concepts of competence behavior. The questions that over $70 \%$ of service providers answered "Always" for were about recognizing cultural barriers and removing those obstacles. Moreover, refugee service providers who have a high level of cultural competence behavior were open to listening to feedback from refugees as well as conworkers.

Table 4-9 Cultural Competence Behavior by Type of Question $(\mathrm{N}=115)$

\begin{tabular}{|c|c|c|c|c|c|c|c|c|}
\hline & \multicolumn{8}{|c|}{ Frequency and Percent of Behavior } \\
\hline & \multicolumn{2}{|c|}{ Never } & \multicolumn{2}{|c|}{ At times } & \multicolumn{2}{|c|}{ Often } & \multicolumn{2}{|c|}{ Always } \\
\hline & $\mathrm{n}$ & $\%$ & $\mathrm{n}$ & $\%$ & $\mathrm{n}$ & $\%$ & $\mathrm{n}$ & $\%$ \\
\hline $\begin{array}{l}\text { I include cultural assessment when I do } \\
\text { refugee evaluations }\end{array}$ & 5 & 4.3 & 13 & 11.3 & 40 & 34.8 & 57 & 49.6 \\
\hline $\begin{array}{l}\text { I seek information on cultural needs } \\
\text { when I identify new refugees in my } \\
\text { practice }\end{array}$ & 0 & 0 & 12 & 10.4 & 28 & 24.3 & 75 & 65.2 \\
\hline $\begin{array}{l}\text { I have resource books and other } \\
\text { materials available to help me learn } \\
\text { about refugees from different cultures }\end{array}$ & 5 & 4.3 & 23 & 20 & 27 & 23.5 & 60 & 50.2 \\
\hline $\begin{array}{l}\text { I use a variety of sources to learn about } \\
\text { the cultural heritage of other people }\end{array}$ & 1 & .9 & 12 & 10.4 & 39 & 33.9 & 63 & 54.8 \\
\hline $\begin{array}{l}\text { I ask refugees to tell me about their } \\
\text { own explanations of problems }\end{array}$ & 3 & 2.6 & 3 & 2.6 & 33 & 28.7 & 76 & 66.1 \\
\hline
\end{tabular}




\begin{tabular}{|c|c|c|c|c|c|c|c|c|}
\hline $\begin{array}{l}\text { I ask refugees to tell me about their } \\
\text { expectations for service }\end{array}$ & 2 & 1.7 & 22 & 19.1 & 43 & 37.4 & 48 & 41.7 \\
\hline $\begin{array}{l}\text { I avoid using generalizations to } \\
\text { stereotype groups of people }\end{array}$ & 0 & 0 & 8 & 7 & 42 & 36.5 & 65 & 56.5 \\
\hline $\begin{array}{l}\text { I recognize potential barriers to service } \\
\text { that might be encountered by different } \\
\text { people }\end{array}$ & 2 & 1.8 & 3 & 2.6 & 28 & 24.3 & 82 & 71.3 \\
\hline $\begin{array}{l}\text { I act to remove obstacles for people of } \\
\text { different cultures when I identify such } \\
\text { obstacles }\end{array}$ & 3 & 2.6 & 0 & 0 & 30 & 26.1 & 82 & 71.3 \\
\hline $\begin{array}{l}\text { I act to remove obstacles for people of } \\
\text { different cultures when refugees } \\
\text { identify such obstacles to me }\end{array}$ & 1 & .9 & 2 & 1.7 & 29 & 25.2 & 83 & 72.2 \\
\hline $\begin{array}{l}\text { I welcome feedback from refugees } \\
\text { about how I relate to others with } \\
\text { different cultures }\end{array}$ & 0 & 0 & 4 & 3.5 & 23 & 20.0 & 88 & 76.5 \\
\hline $\begin{array}{l}\text { I welcome feedback from co-workers } \\
\text { about how I relate to others with } \\
\text { different cultures }\end{array}$ & 0 & 0 & 3 & 2.6 & 21 & 18.3 & 91 & 79.1 \\
\hline $\begin{array}{l}\text { I find ways to adapt my services to } \\
\text { refugees cultural preferences }\end{array}$ & 0 & 0 & 6 & 5.2 & 38 & 33.0 & 71 & 61.7 \\
\hline I document cultural assessment & 16 & 13.9 & 21 & 18.3 & 28 & 24.3 & 50 & 43.5 \\
\hline $\begin{array}{l}\text { I document the adaptations I make with } \\
\text { refugees }\end{array}$ & 18 & 15.7 & 26 & 22.6 & 29 & 25.2 & 42 & 36.5 \\
\hline $\begin{array}{l}\text { I learn from my co-workers about } \\
\text { people with different cultural heritage }\end{array}$ & 1 & .9 & 5 & 4.3 & 24 & 20.9 & 85 & 73.9 \\
\hline
\end{tabular}

Second, the summed cultural awareness and sensitivity (CAS) ranged from 6 to 44 with a mean of $37(S D=4.5)$. The theoretical score range is from 16 to 64 . The scale was considerably reliable with the Cronbach's Alpha coefficient of .78.

Table 4-10 shows detailed cultural awareness/sensitivity by type of question. Question response showed that most of refugee service providers were aware of client culture and culture based service provision. 
Table 4-10 Cultural Awareness/Sensitivity by Type of Question ( $\mathrm{N}=115)$

\begin{tabular}{|c|c|c|c|c|}
\hline & \multicolumn{4}{|c|}{$\begin{array}{c}\text { Frequency and Percent of } \\
\text { Awareness/Sensitivity }\end{array}$} \\
\hline & \multicolumn{2}{|c|}{ Agree } & \multicolumn{2}{|c|}{ Disagree } \\
\hline & $\mathrm{n}$ & $\%$ & $\mathrm{n}$ & $\%$ \\
\hline $\begin{array}{l}\text { Race is the most important factor in } \\
\text { determining a person's culture }\end{array}$ & 20 & 17.4 & 95 & 82.6 \\
\hline $\begin{array}{l}\text { People with a common cultural } \\
\text { background think and act alike }\end{array}$ & 32 & 27.8 & 83 & 72.2 \\
\hline $\begin{array}{l}\text { Many aspects of culture influence } \\
\text { services }\end{array}$ & 106 & 92.2 & 9 & 7.8 \\
\hline $\begin{array}{l}\text { Aspects of cultural diversity need to be } \\
\text { assessed for each individual, group, and } \\
\text { organization }\end{array}$ & 111 & 96.5 & 4 & 3.5 \\
\hline $\begin{array}{l}\text { If I know about a person's culture, I do } \\
\text { not need to assess their personal } \\
\text { preferences for services }\end{array}$ & 8 & 6.9 & 107 & 93.1 \\
\hline $\begin{array}{l}\text { Spirituality and religious beliefs are } \\
\text { important aspects of many cultural } \\
\text { groups }\end{array}$ & 112 & 97.4 & 3 & 2.6 \\
\hline $\begin{array}{l}\text { Individuals may identify with more than } \\
\text { one cultural group }\end{array}$ & 112 & 97.4 & 3 & 2.6 \\
\hline $\begin{array}{l}\text { Language barriers are the only } \\
\text { difficulties for recent refugees to the } \\
\text { United States }\end{array}$ & 12 & 10.4 & 103 & 89.6 \\
\hline $\begin{array}{l}\text { I understand that people from different } \\
\text { cultures may define the concept of } \\
\text { "services" in different ways }\end{array}$ & 113 & 98.2 & 2 & 1.8 \\
\hline $\begin{array}{l}\text { I think that knowing about different } \\
\text { cultural groups helps direct my work } \\
\text { with individuals, families, groups, and } \\
\text { organizations }\end{array}$ & 114 & 99.1 & 1 & .9 \\
\hline $\begin{array}{l}\text { I enjoy working with people who are } \\
\text { culturally different from me }\end{array}$ & 114 & 99.1 & 1 & .9 \\
\hline
\end{tabular}

Organizational Culture Factor. There were three subscales that measured organizational culture where refugee service providers work. First, for the summed proficiency culture, the mean score was $48.7(S D=7.1)$ and the score ranged from 23 to 
60. The theoretical score range was from 15 to 60 . The scale was considerably reliable with the Cronbach's Alpha coefficient of .90 . Second, the summed rigidity culture ranged from 18 to 47 with a mean of $29.0(S D=6.0)$. The theoretical score range was from 11 to 56. The scale was considerably reliable with the Cronbach's Alpha coefficient of .79 . Lastly, mean of the summed resistance culture was $26.0(S D=4.5)$ and the score ranged from 19 to 43 . The theoretical score range was from 13 to 52 . The scale was considerably reliable with the Cronbach's Alpha coefficient of .62.

Table 4-11 showed details of proficiency organization culture by type of question. According to the answer, organizational culture of service participants in this study expect to act in the best interest of each client and to strive for excellence.

Table 4-11 Proficiency Organizational Culture by Type of Question (N=115)

\begin{tabular}{|c|c|c|c|c|c|c|c|c|}
\hline & \multicolumn{8}{|c|}{ Frequency and Percent of Proficiency Culture } \\
\hline & \multicolumn{2}{|c|}{ Not at all } & \multicolumn{2}{|c|}{$\begin{array}{c}\text { A slight } \\
\text { extent }\end{array}$} & \multicolumn{2}{|c|}{$\begin{array}{c}\text { A great } \\
\text { extent }\end{array}$} & \multicolumn{2}{|c|}{$\begin{array}{c}\text { A very } \\
\text { great extent }\end{array}$} \\
\hline & $\mathrm{n}$ & $\%$ & $\mathrm{n}$ & $\%$ & $\mathrm{n}$ & $\%$ & $\mathrm{n}$ & $\%$ \\
\hline $\begin{array}{l}\text { Members of my organizational unit are } \\
\text { expected to improve the well-being of } \\
\text { each client }\end{array}$ & 0 & 0 & 7 & 6.2 & 45 & 39.8 & 61 & 54.0 \\
\hline $\begin{array}{l}\text { Members of my organizational unit are } \\
\text { expected to evaluate how much we } \\
\text { benefit clients }\end{array}$ & 4 & 3.5 & 23 & 20.4 & 50 & 44.2 & 36 & 31.9 \\
\hline $\begin{array}{l}\text { Members of my organizational unit are } \\
\text { expected to place the well-being of } \\
\text { clients' first }\end{array}$ & 1 & .9 & 11 & 9.7 & 41 & 36.3 & 60 & 53.1 \\
\hline $\begin{array}{l}\text { Members of my organizational unit are } \\
\text { expected to find ways to serve clients } \\
\text { more effectively }\end{array}$ & 1 & .9 & 5 & 4.4 & 40 & 35.4 & 67 & 59.3 \\
\hline $\begin{array}{l}\text { Members of my organizational unit are } \\
\text { expected to act in the best interest of } \\
\text { each client }\end{array}$ & 0 & 0 & 7 & 6.2 & 26 & 23.0 & 80 & 70.8 \\
\hline
\end{tabular}




\begin{tabular}{|c|c|c|c|c|c|c|c|c|}
\hline $\begin{array}{l}\text { Members of my organizational unit are } \\
\text { expected to be available to each client } \\
\text { we serve }\end{array}$ & 3 & 2.7 & 21 & 18.8 & 59 & 52.7 & 29 & 25.9 \\
\hline $\begin{array}{l}\text { Members of my organizational unit are } \\
\text { expected to be responsive to the needs } \\
\text { of each client }\end{array}$ & 2 & 1.8 & 7 & 6.2 & 42 & 37.2 & 62 & 54.9 \\
\hline $\begin{array}{l}\text { Members of my organizational unit are } \\
\text { expected to have up-to-date knowledge }\end{array}$ & 1 & .9 & 11 & 9.7 & 47 & 41.6 & 54 & 47.8 \\
\hline $\begin{array}{l}\text { Members of my organizational unit are } \\
\text { expected to become more effective in } \\
\text { serving clients }\end{array}$ & 3 & 2.7 & 11 & 9.8 & 40 & 35.7 & 58 & 51.8 \\
\hline $\begin{array}{l}\text { Members of my organizational unit are } \\
\text { expected to learn new tasks }\end{array}$ & 3 & 2.7 & 19 & 16.8 & 52 & 46.0 & 39 & 34.5 \\
\hline $\begin{array}{l}\text { Members of my organizational unit are } \\
\text { expected to interact positively with } \\
\text { others }\end{array}$ & 2 & 1.8 & 2 & 1.8 & 34 & 30.1 & 75 & 66.4 \\
\hline $\begin{array}{l}\text { Members of my organizational unit are } \\
\text { expected to strive for excellence }\end{array}$ & 1 & .9 & 9 & 8.0 & 24 & 21.2 & 79 & 69.9 \\
\hline $\begin{array}{l}\text { Members of my organizational unit are } \\
\text { expected to be number one }\end{array}$ & 59 & 52.2 & 29 & 25.7 & 15 & 13.3 & 10 & 8.8 \\
\hline $\begin{array}{l}\text { Members of my organizational unit are } \\
\text { expected to plan for success }\end{array}$ & 3 & 2.7 & 15 & 13.3 & 61 & 54.0 & 34 & 30.1 \\
\hline $\begin{array}{l}\text { Members of my organizational unit are } \\
\text { expected to pay attention to detail }\end{array}$ & 1 & .9 & 10 & 8.9 & 51 & 45.5 & 50 & 44.6 \\
\hline
\end{tabular}

Furthermore, Table 4-12 shows details of rigid organization culture by type of question.

Table 4-12 Rigidity Organizational Culture by Type of Question (N=115)

\begin{tabular}{|c|c|c|c|c|c|c|c|c|}
\hline & \multicolumn{8}{|c|}{ Frequency and Percent of Rigidity Culture } \\
\hline & \multicolumn{2}{|c|}{ Not at all } & \multicolumn{2}{|c|}{$\begin{array}{l}\text { A slight } \\
\text { extent }\end{array}$} & \multicolumn{2}{|c|}{$\begin{array}{c}\text { A great } \\
\text { extent }\end{array}$} & \multicolumn{2}{|c|}{$\begin{array}{l}\text { A very } \\
\text { great extent }\end{array}$} \\
\hline & $\mathrm{n}$ & $\%$ & $\mathrm{n}$ & $\%$ & $\mathrm{n}$ & $\%$ & $\mathrm{n}$ & $\%$ \\
\hline $\begin{array}{l}\text { I have to ask a supervisor or } \\
\text { coordinator before I do almost } \\
\text { anything }\end{array}$ & 70 & 61.9 & 30 & 26.5 & 7 & 6.2 & 6 & 5.3 \\
\hline $\begin{array}{l}\text { A person can make his or her own } \\
\text { decisions without checking with } \\
\text { anyone else }\end{array}$ & 17 & 15.2 & 57 & 50.9 & 33 & 29.5 & 5 & 4.5 \\
\hline
\end{tabular}




\begin{tabular}{|c|c|c|c|c|c|c|c|c|}
\hline $\begin{array}{l}\text { Any decision I make has to have a } \\
\text { supervisor's or coordinator's approval }\end{array}$ & 29 & 25.9 & 62 & 55.4 & 12 & 10.7 & 9 & 8.0 \\
\hline $\begin{array}{l}\text { There is only one way to do the job-the } \\
\text { boss's way }\end{array}$ & 82 & 72.6 & 19 & 16.8 & 5 & 4.4 & 7 & 6.2 \\
\hline $\begin{array}{l}\text { Whenever we have a problem, we are } \\
\text { supposed to go to the same person for } \\
\text { an answer }\end{array}$ & 49 & 43.8 & 48 & 42.9 & 12 & 10.7 & 3 & 2.7 \\
\hline $\begin{array}{l}\text { People here always get their orders } \\
\text { from higher up }\end{array}$ & 18 & 16.2 & 63 & 56.8 & 24 & 21.6 & 6 & 5.4 \\
\hline $\begin{array}{l}\text { There can be little action until a } \\
\text { supervisor or coordinator approves the } \\
\text { decision }\end{array}$ & 41 & 36.3 & 53 & 46.9 & 13 & 11.5 & 6 & 5.3 \\
\hline $\begin{array}{l}\text { The same steps must be followed in } \\
\text { processing every piece of work }\end{array}$ & 23 & 20.4 & 39 & 34.5 & 37 & 32.7 & 14 & 12.4 \\
\hline $\begin{array}{l}\text { We usually work under the same } \\
\text { circumstances day to day }\end{array}$ & 59 & 52.2 & 37 & 32.7 & 12 & 10.6 & 5 & 4.4 \\
\hline $\begin{array}{l}\text { People here do the same job on the } \\
\text { same way everyday }\end{array}$ & 59 & 52.2 & 38 & 33.6 & 10 & 8.8 & 6 & 5.3 \\
\hline $\begin{array}{l}\text { How things are done around here is left } \\
\text { pretty much up to the person doing the } \\
\text { work }\end{array}$ & 12 & 10.6 & 47 & 41.6 & 41 & 36.3 & 13 & 11.5 \\
\hline $\begin{array}{l}\text { The same procedures are to be } \\
\text { followed in most situations }\end{array}$ & 11 & 9.7 & 57 & 50.4 & 36 & 31.9 & 9 & 8.0 \\
\hline $\begin{array}{l}\text { I feel that I am my own boss in most } \\
\text { matter }\end{array}$ & 13 & 11.5 & 40 & 35.4 & 38 & 33.6 & 22 & 19.5 \\
\hline $\begin{array}{l}\text { We are to follow strict operating } \\
\text { procedures at all times }\end{array}$ & 10 & 8.9 & 48 & 42.9 & 37 & 33.0 & 17 & 15.2 \\
\hline
\end{tabular}

Table 4-13 showed details of resistance organization culture by type of question.

Table 4-13 Resistance Organizational Culture by Type of Question ( $\mathrm{N}=115)$

\begin{tabular}{|c|c|c|c|c|c|c|c|c|}
\hline & \multicolumn{8}{|c|}{ Frequency and Percent of Resistance Culture } \\
\hline & \multicolumn{2}{|c|}{ Not at all } & \multicolumn{2}{|c|}{$\begin{array}{l}\text { A slight } \\
\text { extent }\end{array}$} & \multicolumn{2}{|c|}{$\begin{array}{l}\text { A great } \\
\text { extent }\end{array}$} & \multicolumn{2}{|c|}{$\begin{array}{l}\text { A very } \\
\text { great extent }\end{array}$} \\
\hline & $\mathrm{n}$ & $\%$ & $\mathrm{n}$ & $\%$ & $\mathrm{n}$ & $\%$ & $\mathrm{n}$ & $\%$ \\
\hline $\begin{array}{l}\text { Members of my organizational unit are } \\
\text { expected to not make waves }\end{array}$ & 52 & 46.4 & 40 & 35.7 & 12 & 10.7 & 8 & 7.1 \\
\hline $\begin{array}{l}\text { Members of my organizational unit are } \\
\text { expected to stay uninvolved }\end{array}$ & 64 & 57.1 & 30 & 26.8 & 11 & 9.8 & 7 & 6.3 \\
\hline
\end{tabular}




\begin{tabular}{lccccccccc}
\hline $\begin{array}{l}\text { Members of my organizational unit are } \\
\text { expected to be stern and unyielding }\end{array}$ & 76 & 67.3 & 24 & 21.2 & 11 & 9.7 & 2 & 1.8 \\
$\begin{array}{l}\text { Members of my organizational unit are } \\
\text { expected to avoid problems }\end{array}$ & 47 & 41.6 & 42 & 37.2 & 16 & 14.2 & 8 & 7.1 \\
$\begin{array}{l}\text { Members of my organizational unit are } \\
\text { expected to follow rather than lead }\end{array}$ & 59 & 52.2 & 40 & 35.4 & 7 & 6.2 & 7 & 6.2 \\
$\begin{array}{l}\text { Members of my organization unit are } \\
\text { expected to be thoughtful and } \\
\text { considerate }\end{array}$ & 0 & 0 & 5 & 4.4 & 27 & 23.9 & 81 & 71.7 \\
$\begin{array}{l}\text { Members of my organizational unit are } \\
\text { expected to be critical }\end{array}$ & 21 & 18.8 & 44 & 39.3 & 35 & 31.3 & 12 & 10.7 \\
$\begin{array}{l}\text { Members of my organizational unit are } \\
\text { expected to avoid being different }\end{array}$ & 81 & 71.7 & 21 & 18.6 & 5 & 4.4 & 6 & 5.3 \\
\hline $\begin{array}{l}\text { Members of my organizational unit are } \\
\text { expected to be dominant and assertive }\end{array}$ & 55 & 49.5 & 36 & 32.4 & 16 & 14.4 & 4 & 3.6 \\
$\begin{array}{l}\text { Members of my organizational unit are } \\
\text { expected to criticize mistakes }\end{array}$ & 40 & 35.7 & 50 & 44.6 & 16 & 14.3 & 6 & 5.4 \\
$\begin{array}{l}\text { Members of my organizational unit are } \\
\text { expected to defeat the competition }\end{array}$ & 83 & 73.5 & 22 & 19.5 & 3 & 2.7 & 5 & 4.4 \\
$\begin{array}{l}\text { Members of my organizational unit are } \\
\text { expected to be competitive with } \\
\text { coworkers }\end{array}$ & 84 & 74.3 & 17 & 15.0 & 8 & 7.1 & 4 & 3.5 \\
$\begin{array}{l}\text { Members of my organizational unit are } \\
\text { expected to go along with group } \\
\text { decisions }\end{array}$ & 14 & 12.4 & 48 & 42.5 & 32 & 28.3 & 19 & 16.8 \\
\hline
\end{tabular}




\subsection{Bivariate Correlation Among the Study Variables}

A summary of the bivariate correlations and significance are presented in Table 414. Pearson's $r$ was used for checking correlation coefficients among variables. Significant indicators on the 12 dependent variables are explained. First, for service provision related dependent variables, correlation results revealed that service provision Domain 1 (orientation) was significantly, positively associated with resettlement specialist $(r=.2, p \leq .05)$, familiarity with community resources $(r=.2, p \leq .05)$, and cultural competence behavior ( $r=.2, p \leq .05)$. Service provision Domain 2 (basic needs) was significantly related to resettlement specialist $(r=.3, p \leq .001)$, familiarity with community resources $(r=.3, p \leq .001)$, and cultural competence behavior $(r=.3, p \leq$ .001 ), and it was a positive relationship with medium level of strength. Service provision domain (assistance to access benefits) was also significantly associated with resettlement specialist $(r=.4, p \leq .001)$, work experience $(r=.2, p \leq .05)$, familiarity with community resources $(r=.3, p \leq .001)$, and cultural competence behavior $(r=.3, p \leq .001)$, and it was a positive relationship. Service provision Domain 4 (employment) was significantly, positively related to familiarity with community resources $(r=.2, p \leq .05)$. Service provision Domain 5 (health) was significantly associated with resettlement specialist ( $r$ $=.2, p \leq .001$ ). Lastly, service provision Domain 6 (other services) was significantly related to resettlement specialist $(r=.3, p \leq .001)$, familiarity with community resources $(r=.3, p \leq .001)$, and cultural competence behavior $(r=.3, p \leq .001)$ with medium level of strength.

Next, for dependent variables related to service referrals, correlation results showed that service referrals Domain 1 (orientation) was positively associated with 
familiarity with community resources $(r=.3, p \leq .001)$. In addition, service referrals Domain 2 (basic needs) was significantly related to work experience $(r=.2, p \leq .05)$, familiarity with community resources $(r=.3, p \leq .001)$, and cultural competence behavior $(r=.3, p \leq .001)$. Service referrals Domain 3 (assistance to access benefits) was significantly, positively correlated to cultural competence behavior $(r=.3, p \leq .001)$. Moreover, service referrals Domain 4 (employment) was significantly associated with resettlement specialist $(r=.2, p \leq .05)$, familiarity with community resources $(r=.3, p \leq$ $.001)$, and cultural competence behavior $(r=.3, p \leq .001)$ with small and medium levels of strength. Service referrals Domain 5 (health) was correlated with familiarity with community resources $(r=.3, p \leq .001)$ and cultural competence behavior $(r=.3, p \leq$ .001). Lastly, Domain 6 (other services) of service referrals was significantly related to non-white $(r=-.2, p \leq .05)$, familiarity with community resources $(r=.2, p \leq .05)$, and cultural competence behavior ( $r=.2, p \leq .001)$. 
Table 4-14 Correlations among Variables

\begin{tabular}{|c|c|c|c|c|c|c|c|c|c|c|c|c|c|c|c|c|c|c|c|c|c|c|c|c|c|}
\hline & 1 & 2 & 3 & 4 & 5 & 6 & 7 & 8 & 9 & 10 & 11 & 12 & 13 & 14 & 15 & 16 & 17 & 18 & 19 & 20 & 21 & 22 & 23 & 24 & 25 \\
\hline 1. Age & 1 & & & & & & & & & & & & & & & & & & & & & & & & \\
\hline 2. $\operatorname{Sex}^{1}$ & 5 & 1 & & & & & & & & & & & & & & & & & & & & & & & \\
\hline 3. Race/Ethnicity ${ }^{2}$ & 5 & $.4 * *$ & 1 & & & & & & & & & & & & & & & & & & & & & & \\
\hline 4. Education ${ }^{3}$ & $.3^{* *}$ & 0 & 0 & 1 & & & & & & & & & & & & & & & & & & & & & \\
\hline 5. Job titelw ${ }^{4}$ & 0 & -.1 & -.1 & . 1 & 1 & & & & & & & & & & & & & & & & & & & & \\
\hline $\begin{array}{l}\text { 6. Work experience } \\
\text { (year) }\end{array}$ & $.4 * *$ & -.0 & .0 & $.2 *$ & 2.2 & 1 & & & & & & & & & & & & & & & & & & & \\
\hline $\begin{array}{l}\text { 7. Knowledge of } \\
\text { refugee }\end{array}$ & $-.2^{*}$ & $.2 *$ & .1 & -.1 & .0 & -.0 & 1 & & & & & & & & & & & & & & & & & & \\
\hline $\begin{array}{l}\text { 8. Familiarity with } \\
\text { community resources }\end{array}$ & -.0 & -.1 & -.2 & .1 & $.2 *$ & $.2 *$ & -.1 & 1 & & & & & & & & & & & & & & & & & \\
\hline $\begin{array}{l}\text { 9. Cultural competence } \\
\text { behavior }\end{array}$ & . 0 & .1 & . 0 & .1 & $.2 *$ & $.2 *$ & 0 & $.5^{* *}$ & 1 & & & & & & & & & & & & & & & & \\
\hline $\begin{array}{l}\text { 10. Cultural awareness } \\
\text { and sensitivity }\end{array}$ & -.2 & $.2 *$ & $.2 *$ & .1 & -.0 & -.1 & .1 & -.0 & $.2^{* *}$ & 1 & & & & & & & & & & & & & & & \\
\hline 11. Proficiency culture & -.1 & -.2 & $-.2^{*}$ & . 0 &,- 1 & 0 & -.1 & .1 & $.3^{* *}$ & 2 & 1 & & & & & & & & & & & & & & \\
\hline 12. Rigidity culture & -.0 & $-.2^{*}$ & -.1 & -.0 & -.1 & .1 & -.1 & . 1 & $.3 * *$ & .1 & $.8^{* *}$ & 1 & & & & & & & & & & & & & \\
\hline 13. Resistance culture & .1 & $-.3 * *$ & $-.2^{*}$ & . 0 & -.1 & 0 & -.2 & $.2 *$ & $.2 *$ & $-.2^{*}$ & $.6^{* *}$ & $.6^{* *}$ & 1 & & & & & & & & & & & & \\
\hline $\begin{array}{l}\text { 14. } \mathrm{SP}^{5} \text { Domain } \\
\text { 1:Orientation }\end{array}$ & -.1 & -.0 & .1 & -.1 & $.2 *$ & .1 & .1 & $.2 *$ & $.2 *$ & -.1 & .1 & .1 & 2 & 1 & & & & & & & & & & & \\
\hline 15. SP D2:Basic needs & -.1 & -.0 & -.0 & -.1 & $.3^{* *}$ & .2 & .1 & $.3^{* *}$ & $.3^{* *}$ & -.1 & .1 & 0 & 0 & $.8^{* *}$ & 1 & & & & & & & & & & \\
\hline
\end{tabular}




\begin{tabular}{|c|c|c|c|c|c|c|c|c|c|c|c|c|c|c|c|c|c|c|c|c|c|c|c|c|c|}
\hline $\begin{array}{l}\text { 16.SP_D3: Assistance } \\
\text { to access benefit }\end{array}$ & -.0 & 1 & 1 & 0 & $.4^{* *}$ & $.2 *$ & -.0 & $3^{* *}$ & $.3^{* *}$ & -.1 & 1 & 1 & . 1 & $.8^{* *}$ & $.8^{* *}$ & 1 & & & & & & & & & \\
\hline $\begin{array}{l}\text { 17. SP_D4: } \\
\text { Employment }\end{array}$ & 0 & .1 & -.0 & -.2 & -.1 & 2 & -.0 & $.2 *$ & 2 & -.0 & .2 & 1 & .1 & $.5^{* *}$ & $.5^{* *}$ & $.4^{* * *}$ & 1 & & & & & & & & \\
\hline 18. SP_D5: Health & -.0 & . 0 & . 1 & . 0 & $.3^{* *}$ & . 1 & 0 & 2 & . 1 & -.2 & . 1 & 1 & .1 & $.7^{* *}$ & $.6^{* *}$ & $.8^{* *}$ & $.3^{* *}$ & 1 & & & & & & & \\
\hline $\begin{array}{l}\text { 19. SP_D6: Other } \\
\text { services }\end{array}$ & -.0 & -.1 & -.1 & -.0 & $.3^{* *}$ & .1 & 0 & $.3^{* *}$ & $.3^{* *}$ & -.1 & 1 & 1 & . 1 & $.7^{* *}$ & $.8 * *$ & $.7^{* *}$ & $.5^{* *}$ & $.6^{* *}$ & 1 & & & & & & \\
\hline $\begin{array}{l}\text { 20. } \mathrm{SR}^{5} \text { Domain 1: } \\
\text { Orientation }\end{array}$ & -.1 & -.1 & -.2 & -.0 & 1 & 0 & -.1 & 2 & $.3^{* *}$ & -.0 & 1 & 1 & 2 & 1 & 1 & 2 & -.0 & 1 & 1 & 1 & & & & & \\
\hline 21. SR_D2: Basic needs & 1 & -.1 & -.2 & . 0 & 2 & $2 *$ & -.1 & $.3^{* *}$ & $.3^{* *}$ & -.0 & .0 & 1 & . 0 & 1 & $.3^{* *}$ & $.2^{* *}$ & . 1 & $.3^{* *}$ & $.3^{* *}$ & $.6^{* *}$ & 1 & & & & \\
\hline $\begin{array}{l}\text { 22. SR_D3: Assistance } \\
\text { to access benefit }\end{array}$ & 0 & -.1 & -.1 & 1 & 1 & 1 & -.0 & 2 & $.3^{* *}$ & 0 & .1 & 0 & 1 & $.3^{* *}$ & $.3^{* *}$ & 2 & . 1 & 2 & $.3^{* *}$ & $.7^{* *}$ & $.8^{* *}$ & 1 & & & \\
\hline $\begin{array}{l}\text { 23. SR_D4: } \\
\text { Employment }\end{array}$ & -.0 & -.1 & -.1 & .0 & $.2 *$ & .1 & -.0 & $.3^{* *}$ & $.3^{* *}$ & -.0 & -.0 & .0 & 0 & $.2^{* *}$ & $.3^{* *}$ & $.3^{* *}$ & . 0 & $.3^{* *}$ & $.3^{* * *}$ & $.5^{* *}$ & $.8^{* *}$ & $.6^{* *}$ & 1 & & \\
\hline 24. SR_D5: Health & -.0 & .0 & -.1 & . 0 & 1 & . 1 & 0 & $.3^{* *}$ & $.3^{* *}$ & 0 & .0 & -.0 & . 1 & $.3 * *$ & $.4 * *$ & $.3 * *$ & . 1 & .2 & $.3 * *$ & $.6^{* *}$ & $.7^{* *}$ & $.8^{* *}$ & $.6^{* *}$ & 1 & \\
\hline $\begin{array}{l}\text { 25. SR_D6: Other } \\
\text { services }\end{array}$ & -.1 & -.1 & $-.2^{*}$ & -.1 & 2 & .1 & 0 & $.2 *$ & $.2^{* *}$ & -.0 & 0 & -.0 & -.0 & $.3^{* *}$ & $.4^{* *}$ & $.3^{* *}$ & .2 & $.4^{* *}$ & $.3^{* *}$ & $.6^{* *}$ & $.8 * *$ & $.7^{* *}$ & $.7^{* *}$ & $.6^{* *}$ & 1 \\
\hline \multicolumn{26}{|c|}{$\begin{array}{l}\text { Note. }{ }^{*} p \leq .05,{ }^{* *} p \leq .01,{ }^{* * *} p \leq .001 \\
{ }^{1} \text { Reference group: female/ } \\
{ }^{2} \text { Reference group: white } \\
{ }^{3} \text { Reference group: Graduate school and above } \\
{ }^{4} \text { Reference group: Resettlement Specialist } \\
{ }^{5} \mathrm{SP}=\text { Service Provided, } \mathrm{SR}=\text { Service Referral }\end{array}$} \\
\hline
\end{tabular}




\subsection{Multiple Regression Analysis}

Multiple regression analysis was conducted to address research questions 2 and 21 regarding the association between service providers' personal and organizational culture factors and service provision and referrals.

Multiple regression results of service provision and referral are presented in Tables 4-15 and 4-16, and six multiple regression analyses for each domain of service provision and referrals are presented in Tables 4-17 and 4-18. Demographics (age, sex, race/ethnicity, education, and job title), personal factors (knowledge of refugee, familiarity with community resources, and cultural competence [cultural competence behavior and cultural awareness/sensitivity]), and organizational culture factors (proficiency, rigidity, and resistance) were treated as independent variables. To parsimonious models, different predictors were entered to the regression for service provision and referrals.

\subsubsection{Multiple Regression Analysis Predicting Overall Service Provision}

First, Table 4-15 presents the results of multiple regression analysis evaluating the personal factors and organizational culture factors to service provision. In the first step, model 1, the control variables were entered as a block to determine the amount of variance they accounted for in service provided. It was found that model 1 was significant, $\mathrm{F}(5,109)=2.26, p=.05$ with $9 \%$ of the variance in service provision accounted for the model 1. Next, model 2, personal factors (knowledge of refugee, familiarity with community resources, and cultural awareness/sensitivity) was added to the regression to assess contribution to service provided. It was significantly related to general service provision, $\mathrm{F}(8,106)=2.91, p=.01$ with $18 \%$ of the variance in service 
provision accounted for by personal factors. The last step, model 3, was to enter proficiency as an organizational culture factor to account for service provided. It was significantly related to general service provision, $\mathrm{F}(9,105)=2.81, p=.01$ with $19 \%$ of the variance in service provision accounted for by organizational culture factors.

In the final model, specifically, service providers who have refugee resettlement related job titles provide more service to refugees than others $(\beta=.24, p=.01)$. In addition, service provision significantly regressed on familiarity with community resources $(\beta=.29, p=.00)$. On the other hand, the proficiency organizational culture factor did not significantly account for variance in overall service provision. 
Table 4-15 Summary of Multiple Regression Analysis for Variables Predicting a Total Number of Service Provision (N=115)

\begin{tabular}{|c|c|c|c|c|c|c|c|c|c|}
\hline \multirow[b]{2}{*}{ Variables } & \multicolumn{3}{|c|}{ Model 1} & \multicolumn{3}{|c|}{ Model 2} & \multicolumn{3}{|c|}{ Model 3} \\
\hline & B & $S E \mathrm{~B}$ & $\beta$ & B & $S E \mathrm{~B}$ & $\beta$ & $\mathrm{B}$ & $S E \mathrm{~B}$ & $\beta$ \\
\hline Age & -.15 & .42 & -.04 & -.12 & .42 & -.03 & -.17 & .42 & -.04 \\
\hline Female (vs. male) & 1.82 & 10.99 & .02 & 2.73 & 10.92 & .03 & 5.95 & 11.01 & .05 \\
\hline White (vs. non-white) & 6.21 & 9.48 & .06 & 11.33 & 9.38 & .12 & 11.67 & 9.35 & .12 \\
\hline Graduate or above (vs. under graduate) & -8.38 & 8.59 & -.10 & -10.17 & 8.39 & -.12 & -9.94 & 8.36 & -.11 \\
\hline Resettlement Specialist (vs. others) & 26.14 & 8.17 & $.29^{* *}$ & 21.26 & 8.02 & $.24 * *$ & 21.67 & 8.00 & $.24 * *$ \\
\hline \multicolumn{10}{|l|}{ Personal Factors } \\
\hline Knowledge of Refugee & & & & 2.11 & 4.37 & .05 & 2.48 & 4.36 & .02 \\
\hline Familiarity with Community Resource & & & & 1.21 & .38 & $.29 * *$ & 1.20 & .38 & $.29 * *$ \\
\hline CAS & & & & -.89 & .91 & -.09 & -1.35 & .58 & -.14 \\
\hline \multicolumn{10}{|l|}{ Organizational Factors } \\
\hline Proficiency & & & & & & & .77 & .58 & .13 \\
\hline $\mathrm{R}^{2}$ & & $.09 *$ & & & $18 * *$ & & & $.19^{* *}$ & \\
\hline
\end{tabular}

Note. ${ }^{+} p \leq .10,{ }^{*} p \leq .05,{ }^{* *} p \leq .01, * * * p \leq .001$ 
Next, Table 4-16 presents the results of multiple regression analysis evaluating the personal factors and organizational culture factors to service referral. In the first step, model 1 , the demographic variables such as age, sex, race/ethnicity, education level, and job title were entered as a block to determine the amount of variance they accounted for in service referral. It was found that the model 1 was not significant, $\mathrm{F}(5,109)=1.19, p$ $=.32$. Next, model 2, cultural competence behavior $(\mathrm{CCB})$ as a personal factor was added to the regression to assess contribution to service referral. It was significantly related to general service provision, $\mathrm{F}(6,108)=3.69, p=.002$ with $17 \%$ of the variance in service referral accounted for by CCB. The last step, model 3, was to enter organizational culture factor (proficiency, rigidity, and resistance) to account for service referral. It was found that the overall model was significantly related to general service referral, $\mathrm{F}(9,105)$ $=2.86, p=.005$ with $20 \%$ of the variance in service provision accounted for by organizational culture factors.

In the final model, specifically, service referral was regressed on race/ethnicity in the demographic variables. Service providers who are non-white was significant in explaining overall service referral to refugees compared to white service providers ( $\beta=$ $-.17, p=.08)$. In addition, service referral significantly associated with $\mathrm{CCB}(\beta=.41, p$ $=.000)$. On the other hand, organizational culture factor did not significantly account for variance in overall service referral. 
Table 4-16 Summary of Multiple Regression Analysis for Variables Predicting a Total Number of Service Referral (N=115)

\begin{tabular}{|c|c|c|c|c|c|c|c|c|c|}
\hline \multirow[b]{2}{*}{ Variables } & \multicolumn{3}{|c|}{ Model 1} & \multicolumn{3}{|c|}{ Model 2} & \multicolumn{3}{|c|}{ Model 3} \\
\hline & B & $S E \mathrm{~B}$ & $\beta$ & B & $S E \mathrm{~B}$ & $\beta$ & $\mathrm{B}$ & $S E \mathrm{~B}$ & $\beta$ \\
\hline Age & -.14 & .35 & -.04 & -.14 & .33 & -.04 & -.16 & .33 & -.05 \\
\hline Female (vs. male) & -1.17 & 9.24 & -.01 & -4.33 & 8.72 & -.05 & -8.52 & 9.08 & -.09 \\
\hline White (vs. non-white) & -9.97 & 7.97 & -.13 & -10.18 & 7.49 & -.13 & -13.71 & 7.76 & $-.17 \dagger$ \\
\hline Graduate or above (vs. under graduate) & -.70 & 7.22 & -.01 & -2.72 & 6.80 & -.04 & -2.55 & 6.79 & -.04 \\
\hline Resettlement Specialist (vs. others) & 12.69 & 6.87 & $.17+$ & 7.07 & 6.61 & .10 & 4.67 & 6.75 & .06 \\
\hline \multicolumn{10}{|l|}{ Personal Factors } \\
\hline $\mathrm{CCB}$ & & & & 1.81 & .46 & $.36 * * *$ & 2.07 & .51 & $.41 * * *$ \\
\hline \multicolumn{10}{|l|}{ Organizational Factors } \\
\hline Proficiency & & & & & & & -.25 & .48 & -.05 \\
\hline Rigidity & & & & & & & -.73 & .71 & -.12 \\
\hline Resistance & & & & & & & -.52 & .96 & -.07 \\
\hline $\mathrm{R}^{2}$ & & .05 & & & $.17^{* *}$ & & & $.20 * *$ & \\
\hline
\end{tabular}

Note. ${ }^{\dagger} p \leq .10,{ }^{*} p \leq .05,{ }^{* *} p \leq .01,{ }^{* * *} p \leq .001$ 


\subsubsection{Multiple Regression Analysis Predicting Domains of Service Provision}

Table 4-17 presents the results of multiple regression analyses evaluating the personal factors and organizational culture factors to domains of service provision. In the first step for each regression, the control variables were entered as a block to determine the amount of variance they accounted for in service provided. Next, personal factors, knowledge of refugee, familiarity with community resources, and cultural awareness/sensitivity were entered to assess contribution to service provided. The last step was to enter proficiency as an organizational culture factor to account for service provided.

Domain 1 (Orientation) Analysis for Service Provision. In the final model, all of the variables were entered as predictors of orientation service provision. In this model, orientation service provision was regressed on two predictors, race/ethnicity and service provider's job title in the demographic variables. The standardized regression coefficients revealed that refugee service providers who are white was significant in explaining orientation service provision to refugees compared to non-white service providers ( $\beta$ $=.17, p=.09$ ). In addition, service providers who have refugee resettlement related job titles provide more orientation service to refugees than others $(\beta=.19, p=.04)$. In the next step, evaluating personal factors, orientation service provision significantly regressed on knowledge of refugee $(\beta=.17, p=.08)$ and familiarity with community resources $(\beta=.18, p=.05)$. On the other hand, proficiency organizational culture factor did not significantly account for variance in orientation service provision.

The overall model significantly accounted for $15 \%$ variances $\left(R^{2}\right)$ in orientation service provision to refugees, $\mathrm{F}(9,105)=2.08, p=.04$. 
Domain 2 (Basic Needs) Analysis for Service Provided. Service providers' level of education $(\beta=-.17, p=.06)$ and job title $(\beta=.31, p=.001)$ in the block of control variables significantly contribute variance to basic needs service provision.

In the personal factor variables, basic needs service provision was regressed on the familiarity with community resources $(\beta=.29, p=.002)$. In contrast, there was no significant association between proficiency organizational culture factor and basic needs service provision to refugees.

The overall model explained $24 \%$ of variances $\left(R^{2}\right)$ in basic needs service provision, $\mathrm{F}(9,105)=3.73, p=.000$.

Domain 3 (Assistance to Access Benefits) Analysis for Service Provided. Service providers' job title $(\beta=.30, p=.001)$ in the block of demographic variables significantly associated with assistance to access benefit service provision. In the personal factor variables, assistance to access benefit service provision was only regressed on the familiarity with community resources $(\beta=.27, p=.003)$. In contrast, there was no significant association between proficiency organizational culture factor and basic needs service provision to refugee.

The overall model explained $21 \%$ of variances $\left(R^{2}\right)$ in assistance to access benefit service provision, $\mathrm{F}(9,105)=3.16, p=.002$.

Domain 4 (Employment) Analysis for Service Provided. Service providers' level of education $(\beta=-.24, p=.02)$ negatively associated with employment service provision among the demographic variables. In the personal factor variables, familiarity with community resources associated with employment service provision $(\beta=.25, p$ 
$=.01)$. Moreover, employment service provision was regressed on proficiency organizational culture factor in the block of organizational factors $(\beta=.19, p=.05)$.

The overall model accounted for $15 \%$ variances $\left(R^{2}\right)$ in employment service provision, $\mathrm{F}(9,105)=2.01, p=.045$.

Domain 5 (Health) Analysis for Service Provided. In the demographic variables, service providers' job title significantly associated with health service provision $(\beta=.26, p=.006)$. The only variable, cultural awareness/sensitivity (CAS), was negatively associated with health service provision $(\beta=-.25, p=.019)$ in the personal factors. In contrast, there was no association between proficiency organizational culture factor and health service provision to refugees.

The overall model accounted for $15 \%$ variances $\left(R^{2}\right)$ in health service provision, $\mathrm{F}$ $(9,105)=2.09, p=.037$.

Domain 6 (Other Services) Analysis for Service Provided. Service providers' job title significantly associated with other service provision in the block of demographics $(\beta=.21, p=.028)$. In the personal factors, other service provision was regressed on service providers' familiarity with community resources $(\beta=.27, p=.005)$. Similar to other domains' regression results, there was no association between proficiency organizational culture factor and other service provision to refugees.

The overall model explained $15 \%$ of variances $\left(R^{2}\right)$ in other service provision, $\mathrm{F}$ $(9,105)=2.09, p=.037$. 
Table 4-17 Summary of Multiple Regression Analysis for Variables Predicting a Number of Service Provision by Domain (N=115)

\begin{tabular}{|c|c|c|c|c|c|c|c|c|c|c|c|c|c|c|c|c|c|c|}
\hline \multirow[b]{2}{*}{ Variables } & \multicolumn{3}{|c|}{ Orientation } & \multicolumn{3}{|c|}{ Basic Needs } & \multicolumn{3}{|c|}{$\mathbf{A A B}^{1}$} & \multicolumn{3}{|c|}{ Employment } & \multicolumn{3}{|c|}{ Health } & \multicolumn{3}{|c|}{ Other Services } \\
\hline & B & $\begin{array}{c}S E \\
\mathrm{~B}\end{array}$ & $\beta$ & B & $\begin{array}{c}S E \\
\mathrm{~B}\end{array}$ & $\beta$ & B & $\begin{array}{c}S E \\
\mathrm{~B}\end{array}$ & $\beta$ & B & $\begin{array}{c}S E \\
\mathrm{~B}\end{array}$ & $\beta$ & B & \begin{tabular}{|c|}
$S E$ \\
$\mathrm{~B}$
\end{tabular} & $\beta$ & B & $\begin{array}{c}S E \\
\mathrm{~B}\end{array}$ & $\beta$ \\
\hline Age & -.01 & .02 & -.05 & -.03 & .05 & -.06 & -.02 & .03 & -.06 & .02 & .02 & .11 & -.02 & .02 & -.08 & .00 & .02 & .02 \\
\hline Female (vs. male) & -.25 & .41 & -.06 & .50 & 1.32 & .04 & .86 & .72 & .12 & .60 & .43 & .14 & .52 & .58 & .69 & -.02 & .54 & -.00 \\
\hline $\begin{array}{l}\text { White (vs. non- } \\
\text { white) }\end{array}$ & .59 & .35 & $.17^{+}$ & .32 & 1.13 & .03 & .67 & .61 & .11 & -.01 & .36 & -.00 & .62 & .49 & .13 & -.06 & .46 & -.01 \\
\hline $\begin{array}{l}\text { Graduate or above } \\
\text { (vs. under } \\
\text { graduate) }\end{array}$ & -.37 & .31 & -.11 & -1.88 & 1.01 & $-.17 \dagger$ & -.22 & .54 & -.04 & -.79 & .32 & $-.24 *$ & -.02 & .44 & -.00 & -.38 & .41 & -.09 \\
\hline $\begin{array}{l}\text { Resettlement } \\
\text { Specialist (vs. } \\
\text { others) }\end{array}$ & .62 & .30 & $.19 *$ & 3.42 & .96 & $.31 * * *$ & 1.74 & .52 & $.30 * * *$ & -.30 & .31 & -.09 & 1.17 & .42 & $.26^{* *}$ & .88 & .39 & $.21 *$ \\
\hline \multicolumn{19}{|l|}{ Personal Factors } \\
\hline $\begin{array}{l}\text { Knowledge of } \\
\text { refugee }\end{array}$ & .29 & .16 & $.17^{\dagger}$ & .37 & .53 & .06 & -.04 & .28 & -.01 & .02 & .17 & .01 & .01 & .23 & .00 & .12 & .22 & .05 \\
\hline $\begin{array}{l}\text { Familiarity with } \\
\text { community } \\
\text { resources }\end{array}$ & .03 & .01 & $.18^{+}$ & .15 & .05 & $.29 * *$ & .09 & .03 & $.27 * *$ & .04 & .02 & $.25^{*}$ & .03 & .02 & .15 & .05 & .02 & $.27^{* *}$ \\
\hline CAS & -.05 & .04 & -.15 & -.12 & .12 & -.10 & -.09 & .06 & -.13 & -.04 & .04 & -.10 & -.12 & .05 & $-.25^{*}$ & -.04 & .05 & -.08 \\
\hline \multicolumn{19}{|c|}{ Organizational Factors } \\
\hline Proficiency & .03 & .02 & .13 & .06 & .07 & .80 & .04 & .04 & .11 & .04 & .02 & $.19 *$ & .04 & .03 & .18 & .03 & .03 & .11 \\
\hline$R^{2}$ & & $.15^{*}$ & & & $.24 * *$ & & & $.21 * *$ & & & $.15^{*}$ & & & $.15 *$ & & & $15^{*}$ & \\
\hline \multicolumn{19}{|c|}{ Note. ${ }^{\dagger} p \leq .10, * p \leq .05, * * p \leq .01, * * * p \leq .001$} \\
\hline Assistance to A & s Bene & - 4 & & & & & & & & & & & & & & & & \\
\hline
\end{tabular}


4.5.3. Multiple Regression Analysis Predicting Domains of Service Referrals

Table 4-18 presents the summary of results of multiple regression analyses evaluating the personal factors and organizational culture factors to domains of service referrals. In the first step for each regression, the control variables (age, sex, race/ethnicity, education, and job title) were entered as a block to determine the amount of variance they accounted for in service referrals. Next, personal factors, cultural competence behavior was entered to assess contribution to service referrals. Lastly, organizational factors, proficiency, rigidity, and resistance were entered to understand their unique contribution in service referrals.

Domain 1 (Orientation) Analysis for Service Referrals. None of the demographic variables found significant association with orientation service referrals. In the next step, personal factor, cultural competence behavior (CCB) was positively associated with orientation service referral $(\beta=.32, p=.003)$. In contrast, there was no association between organizational culture factors and orientation service referral to refugees.

The overall model accounted for $14 \%$ variances $\left(R^{2}\right)$ in orientation service referral, $\mathrm{F}(9,105)=1.93, p=.06$.

Domain 2 (Basic Needs) Analysis for Service Referrals. In the demographic variables, race/ethnicity negatively associated with basic needs service referrals $(\beta=-.22$, $p=.03)$. Cultural competence behavior (CCB) was significantly associated with basic needs service referrals $(\beta=.34, p=.001)$ in the personal factors. For organizational culture factors, there was no association between proficiency, rigidity, and resistance and basic needs service referrals to refugees. 
The overall model explained $19 \%$ of the variances $\left(R^{2}\right)$ in basic needs service referral, $\mathrm{F}(9,105)=2.79, p=.01$.

\section{Domain 3 (Assistance to Access Benefits) Analysis for Service Referrals. In}

the final model, demographics, personal factor, and organizational culture factor variables accounted for $14 \%$ of the variances $\left(R^{2}\right)$ in the assistance to access benefits service referrals $(\mathrm{F}(9,105)=1.96, p=.05)$.

In the demographic variables, race/ethnicity $(\beta=-.17, p=.09)$ negatively associated with assistance to access benefits service referrals. Cultural competence behavior (CCB) was significantly associated with assistance to access benefits service referrals $(\beta=.32, p=.00)$ in the personal factors. For organizational culture factors, rigid organizational culture $(\beta=-.23, p=.07)$ was negatively associated with assistance to access benefits. However, the other two organizational culture factors were not significant predictors to assistance to access benefits service referrals to refugees.

Domain 4 (Employment) Analysis for Service Referrals. None of the demographic variables found significant association between employment service referrals. Similar to the findings for other domain regression results, cultural competence behavior (CCB) was positively associated with employment service referrals $(\beta=.26, p$ $=.02)$. For organizational culture factors, rigid organizational culture $(\beta=-.23, p=.07)$ was negatively associated with assistance to access benefits service referrals. In contrast, there was no association between the other organizational culture factors and employment service referral to refugees.

The overall model explained $15 \%$ of variances $\left(R^{2}\right)$ in employment service referral, $\mathrm{F}(9,105)=2.05, p=.04$. 
Domain 5 (Health) Analysis for Service Referrals. None of the demographic variables found significant association between health service referrals. In the personal factors, health service referrals was regressed on cultural competence behavior $(\beta=.36, p$ $=.001)$. In contrast, there was no association between organizational culture factors and health service referrals to refugees.

The final model did not significantly explain health service referrals.

Domain 6 (Other Services) Analysis for Service Referrals. In the final model, demographics, personal factor, and organizational culture factor variables accounted for $15 \%$ of the variances $\left(R^{2}\right)$ in other service referral $(\mathrm{F}(9,105)=2.10, p=.04)$.

Race/ethnicity (white; $\beta=-.24, p=.02$ ) negatively associated with other service referrals. Other service referrals was regressed on cultural competence behavior (CCB; $\beta$ $=.28, p=.01)$. For organizational culture factors, none of the variables associated with other service referral. 
Table 4-18 Summary of Regression Analysis for Variables Predicting a Number of Service Referral by Domain (N=115)

\begin{tabular}{|c|c|c|c|c|c|c|c|c|c|c|c|c|c|c|c|c|c|c|}
\hline \multirow[b]{2}{*}{ Variables } & \multicolumn{3}{|c|}{ Orientation } & \multicolumn{3}{|c|}{ Basic Needs } & \multicolumn{3}{|c|}{$\mathbf{A A B}^{1}$} & \multicolumn{3}{|c|}{ Employment } & \multicolumn{3}{|c|}{ Health } & \multicolumn{3}{|c|}{ Other Services } \\
\hline & B & $\begin{array}{c}S E \\
\mathrm{~B}\end{array}$ & $\beta$ & B & $\begin{array}{c}S E \\
\mathrm{~B} \\
\end{array}$ & $\beta$ & B & $\begin{array}{c}S E \\
\mathrm{~B}\end{array}$ & $\beta$ & B & $\begin{array}{c}S E \\
\mathrm{~B}\end{array}$ & $\beta$ & B & $\begin{array}{c}S E \\
\mathrm{~B} \\
\end{array}$ & $\beta$ & B & $\begin{array}{c}S E \\
\mathrm{~B} \\
\end{array}$ & $\beta$ \\
\hline Age & -.02 & .01 & -.13 & .02 & .04 & .05 & -.00 & .02 & -.01 & -.01 & .01 & -.04 & -.01 & .02 & -.04 & -.02 & .02 & -.08 \\
\hline Female (vs. male) & -.15 & .35 & -.05 & -1.16 & 1.14 & -.10 & -.57 & .67 & -.09 & -.45 & .38 & -.12 & -.05 & .55 & -.01 & -.21 & .55 & -.04 \\
\hline $\begin{array}{l}\text { White (vs. non- } \\
\text { white) }\end{array}$ & -.43 & .30 & -.14 & -2.19 & .97 & $-.22^{*}$ & -.98 & .57 & $-.17 \dagger$ & -.47 & .33 & -.15 & -.56 & .47 & -.12 & -1.09 & .47 & $-.24^{*}$ \\
\hline $\begin{array}{l}\text { Graduate or above } \\
\text { (vs. under } \\
\text { graduate) }\end{array}$ & -.02 & .26 & .05 & -.08 & .85 & -.01 & .15 & .50 & .03 & .04 & .28 & .01 & .07 & .41 & .02 & -.37 & .41 & -.09 \\
\hline $\begin{array}{l}\text { Resettlement } \\
\text { Specialist (vs. } \\
\text { others) }\end{array}$ & .13 & .26 & .05 & .58 & .85 & .06 & -.06 & .50 & -.01 & .31 & .28 & .10 & .11 & .41 & .03 & .33 & .41 & .08 \\
\hline \multicolumn{19}{|l|}{ Personal Factors } \\
\hline $\mathrm{CCB}$ & .00 & .02 & $.32 * *$ & .22 & .07 & $.34 * * *$ & .12 & .04 & $.32 * *$ & .05 & .02 & $.26^{*}$ & .11 & .03 & $.36 * * *$ & .08 & .03 & $.28 * *$ \\
\hline \multicolumn{19}{|c|}{ Organizational Factors } \\
\hline Proficiency & -.01 & .02 & -.08 & .01 & .06 & .02 & -.01 & .04 & -.03 & .00 & .02 & .02 & -.03 & .03 & -.10 & -.01 & .03 & -.04 \\
\hline Rigidity & -.01 & .03 & -.04 & -.12 & .09 & -.16 & -.10 & .05 & $-.23+$ & -.06 & .03 & $-.23+$ & -.02 & .04 & -.07 & -.05 & .04 & -.16 \\
\hline Resistance & .02 & .04 & .06 & -.06 & .12 & -.06 & .05 & .07 & .09 & -.01 & .04 & -.02 & -.01 & .06 & -.03 & -.02 & .06 & -.04 \\
\hline $\mathrm{R} 2$ & & $.14 \dagger$ & & & $.19^{* *}$ & & & $.14 *$ & & & $.15^{*}$ & & & .13 & & & $.15^{*}$ & \\
\hline
\end{tabular}




\subsection{Mediation}

Mediation analyses were conducted in SPSS Macro to address research questions three and four regarding the cultural competence mediation effect on the association between service providers' familiarity with community resources/work experience and each domain of service provision and referrals.

All mediation models illustrate the following: effects (represented as unstandardized coefficients) of the independent variable (familiarity with community resources and work experience) on the proposed mediator variable (cultural competence; a path); the effects of the mediator variables on the dependent variable (each domain of service provided and referral) taking the other mediators into account (b path); the total effects; the direct effect (c' path); and the specific indirect effects ( $a^{*} b$ paths).

Figure 4-1 and Table 4-19 indicate the overall cultural competence (cultural competence behavior and cultural awareness/sensitivity) mediation effect on the association between service providers' familiarity with community resources and service provision.

Very little of the variance in cultural awareness/sensitivity (CAS) is explained by the familiarity with community resources $\left(\mathrm{R}^{2}=.00\right)$, and this path was not statistically significant. In contrast, cultural competence behavior (CCB) is well explained by familiarity with community resources $\left(\mathrm{R}^{2}=.22\right)$, and it was statistically significant.

The indirect effect of familiarity with community resources on service provision through CCB is the product of the effect of familiarity with community resources on CCB ( $a_{1}$ from Figure 4-1) and the effect of CCB on service provision ( $b_{1}$ from Figure 41), holding all else constant. That is $\mathrm{a}_{1} \mathrm{~b}_{1}=(.32) *(1.69)=.54$. So, level of familiarity 
with community resources seems to increase service provision by .54 units indirectly, through its effect on increasing CCB. The confidence interval of 95\% was from .17 to 1.08. The CI (95\%), not including zero, indicates significant indirect effects and, thus, that the effect of the familiarity with community resource on service provision is mediated by the cultural competence behavior.

The indirect effects of familiarity with community resources on service provision through CAS include zero, a 95\% confidence interval from -.06 to .17. It indicates no significant indirect effects.

The direct effect of familiarity with community resources, $c^{\prime}=.72$, is the estimated difference in service provision between two service providers experiencing the same level of cultural competence behavior but who differ by one unit in their reported familiarity with community resources. However, this direct effect is not statistically different from zero, $\mathrm{t}(111)=1.72, \mathrm{p}=.089$, with a $95 \%$ confidence interval from -.11 to 1.55 .

Observe that the total effect $\mathrm{C}$ is the sum of the direct effect of familiarity with community resources and the two indirect effects of familiarity with community resources, through CCB and CAS. That is, $\mathrm{C}=\mathrm{c}^{\prime}+\mathrm{a}_{1} \mathrm{~b}_{1}+\mathrm{a}_{2} \mathrm{~b}_{2}=(.72)+(.54)+(.02)=1.28$. Service providers who differ by one unit in familiarity with community resources are estimated to differ by 1.28 units in their reported service provision. The positive sign means persons who have more familiarity with community resources report providing more service to refugees ( $95 \%$ confidence interval from .53 to 2.01 ). 
$\mathrm{X}$ : Familiarity with Community Resources

Y: Service Provision

$\mathrm{M}_{1}$ : Cultural Competence Behavior

$\mathrm{M}_{2}$ : Cultural Awareness/Sensitivity

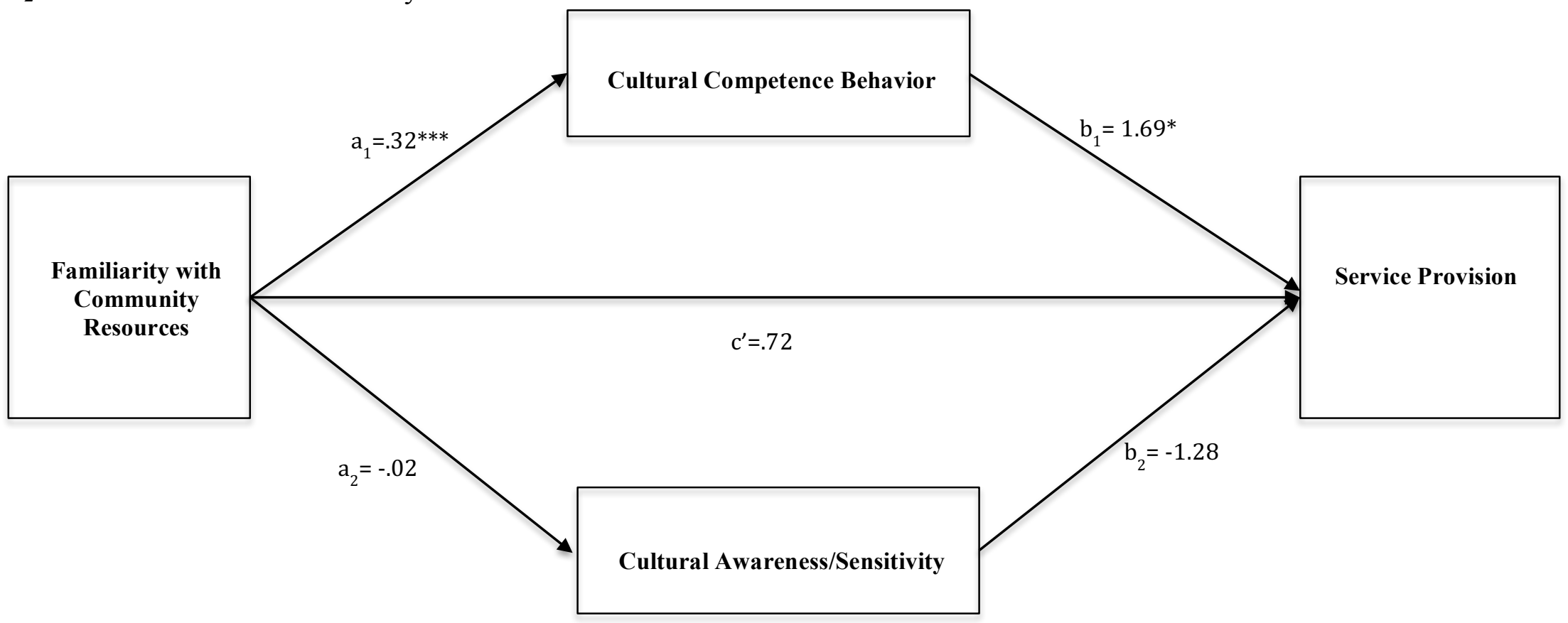

Figure 4-1 Parallel Multiple Mediator Model 
Table 4-19 Regression Coefficients, Standards Errors, and Model Summary Information for the Parallel Multiple Mediator Model Depicted in Figure 4-1.

\begin{tabular}{|c|c|c|c|c|c|c|c|c|c|c|c|c|}
\hline & & \multicolumn{11}{|c|}{ Consequent } \\
\hline & & \multicolumn{3}{|c|}{$\begin{array}{c}\mathrm{M}_{1} \\
\text { (Cultural Competence Behavior) }\end{array}$} & & \multicolumn{3}{|c|}{$\begin{array}{c}\mathrm{M}_{2} \\
(\text { Cultural } \\
\text { Awareness/Sensitivity })\end{array}$} & & \multicolumn{3}{|c|}{$\begin{array}{c}\text { Y } \\
\text { (Service Provision) }\end{array}$} \\
\hline \multicolumn{2}{|l|}{ Antecedent } & Coeff. & $S E$ & $\mathrm{p}$ & & Coeff. & $S E$ & $\mathrm{p}$ & & Coeff. & $S E$ & $\mathrm{p}$ \\
\hline$X$ & \multirow[t]{3}{*}{$a_{1}$} & .32 & .06 & .000 & $\mathrm{a}_{2}$ & -.02 & .04 & .71 & $c^{\prime}$ & .72 & .42 & .09 \\
\hline $\mathrm{M}_{1}$ & & & & & & & & & $b_{1}$ & 1.69 & .64 & .01 \\
\hline $\mathrm{M}_{2}$ & & & & & & & & & $\mathrm{~b}_{2}$ & -1.28 & .89 & .15 \\
\hline \multirow[t]{3}{*}{ Constant } & $\mathrm{i}_{\mathrm{M} 1}$ & 35.03 & 3.63 & .000 & $\mathrm{i}_{\mathrm{M} 2}$ & 37.93 & 2.63 & .000 & $\mathrm{I}_{\mathrm{Y}}$ & -28.40 & 41.82 & .50 \\
\hline & & \multicolumn{3}{|c|}{$\mathrm{R}^{2}=.22$} & & \multicolumn{3}{|c|}{$\mathrm{R}^{2}=.001$} & & \multicolumn{3}{|c|}{$\mathrm{R}^{2}=.15$} \\
\hline & & \multicolumn{3}{|c|}{$F(1,113)=31.99 p=.000$} & & \multicolumn{3}{|c|}{$F(1,113)=.14, p=.71$} & & \multicolumn{3}{|c|}{$F(3,111)=6.50, p=.00$} \\
\hline
\end{tabular}


Figure 4-2 indicates a cultural competence (cultural competence behavior and cultural awareness/sensitivity) mediation effect on the association between service providers' familiarity with community resources and service provision by six domains. The mediation effects of cultural competence behavior were founded on the association between familiarity with community resources and basic needs (Domain 2), assistance to access benefits (Domain 3), and other service (Domain 6) service provision through CCB.

For basic needs service provision, $\mathrm{a}_{1} \mathrm{~b}_{1}=(.32) *(.18)=.06$. So, the level of familiarity with community resources seems to increase basic needs service provision by .06 units, indirectly, through its effect on increasing $\mathrm{CCB}$, which in turn leads to higher basic needs service provision. The 95\% confidence interval is from .01 to .12.

Next, level of familiarity with community resources increases assistance to access to benefits service provision by .03 units $\left(a_{1} b_{1}=(.32) *(.09)=.03\right)$, indirectly, through its effect on increasing $\mathrm{CCB}$, which in turn leads to higher assistance to access benefits. The $95 \%$ confidence interval is from .001 to .06 .

Lastly, other service provision was significantly mediated by familiarity with community resources through $\mathrm{CCB}$ (95\% confidence interval is from .001 to .04). CI (95\%) not including zero indicates significant indirect effects and, thus, that the effect of the familiarity with community resources on other service provision was mediated by the cultural competence behavior.

There were no indirect effects of familiarity with community resources on basic needs, assistance to access benefits, and other service provision through cultural awareness/sensitivity (CAS). Thus, the effect of the familiarity with community resources 
on basic needs, assistance to access benefits, and other service provision were not mediated by CAS.

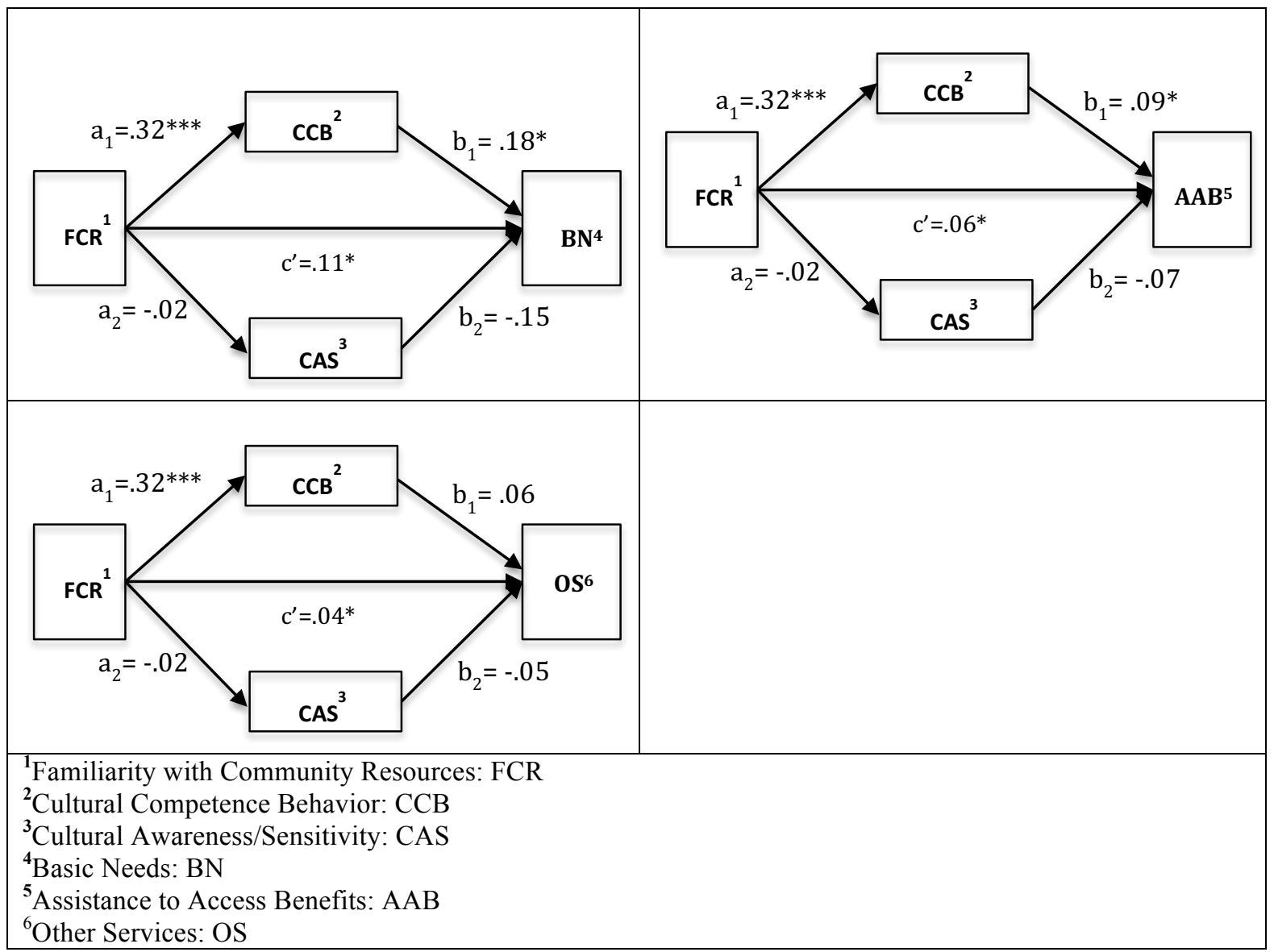

Figure 4-2 Cultural Competence Mediate Association between Familiarity with Community Resources and Service Provision by Six Domains

Figure 4-3 and Table 4-20 indicate the overall cultural competence (cultural competence behavior and cultural awareness/sensitivity) mediation effect on the association between service providers' familiarity with community resources and service referrals.

CAS is explained by the familiarity with community resources $\left(\mathrm{R}^{2}=.001\right)$, which is not significant; CCB is well explained by the familiarity with community resources 
$\left(\mathrm{R}^{2}=.22\right)$. More specifically, the indirect effect of familiarity with community resources on service referrals through $\mathrm{CCB}$ is the product of the effect of familiarity with community resources on $\mathrm{CCB}$ ( $\mathrm{a}_{1}$ from figure) and the effect of $\mathrm{CCB}$ on service referrals $\left(\mathrm{b}_{1}\right.$ from figure), holding all else constant. That is, $\mathrm{a}_{1} \mathrm{~b}_{1}=(.32) *(1.58)=.51$. So, level of familiarity with community resources seems to increase service referrals by .51 units, indirectly, through its effect on increasing $\mathrm{CCB}$, which in turn leads to higher service provision. The $95 \%$ confidence interval is from .22 to .93 . CI (95\%) not including zero indicates significant indirect effects and, thus, that the effect of the familiarity with community resource on service referrals is mediated by the cultural competence behavior.

The indirect effects of familiarity with community resources on service referrals through CAS is not statistically different from zero ( $95 \%$ confidence interval from -1.92 to .96). Thus, the effect of the familiarity with community resources on service referrals was not mediated by CAS.

The direct effect of familiarity with community resources, $\mathrm{c}^{\prime}=.50$, is the estimated difference in service referrals between two service providers experiencing the same level of cultural competence behavior but who differ by one unit in their reported familiarity with community resources. However, this direct effect is not statistically different from zero, $\mathrm{t}(111)=1.44, \mathrm{p}=.15$, with a $95 \%$ confidence interval from -.18 to 1.18 .

Observe that the total effect $\mathrm{C}$ is the sum of the direct effect of familiarity with community resources and the two indirect effects of familiarity with community resources, through CCB and CAS. That is, $\mathrm{C}=\mathrm{c}^{\prime}+\mathrm{a}_{1} \mathrm{~b}_{1}+\mathrm{a}_{2} \mathrm{~b}_{2}=(.50)+(.51)+(.01)=1.02$. Service providers who differ by one unit in familiarity with community resources are estimated to differ by 1.02 units in their reported service referrals. The positive sign 
means the persons who have more familiarity with community resources report more referred service to refugees (95\% confidence interval from .39 to 1.61$)$. 
X: Familiarity with Community Resources

Y: Service Referrals

$\mathrm{M}_{1}$ : Cultural Competence Behavior

$\mathrm{M}_{2}$ : Cultural Awareness/Sensitivity

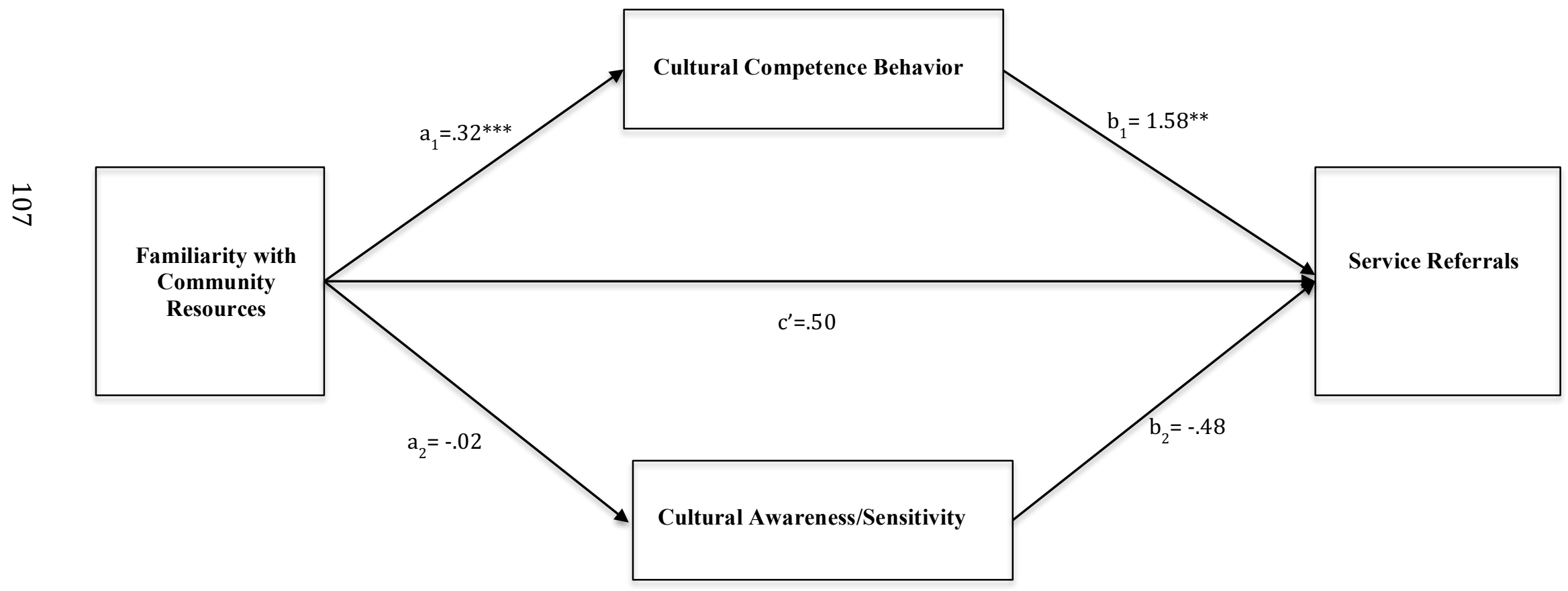

Figure 4-3 Parallel Multiple Mediator Model 2 
Table 4-20 Regression Coefficients, Standards Errors, and Model Summary Information for the Parallel Multiple Mediator Model Depicted in Figure 4-2.

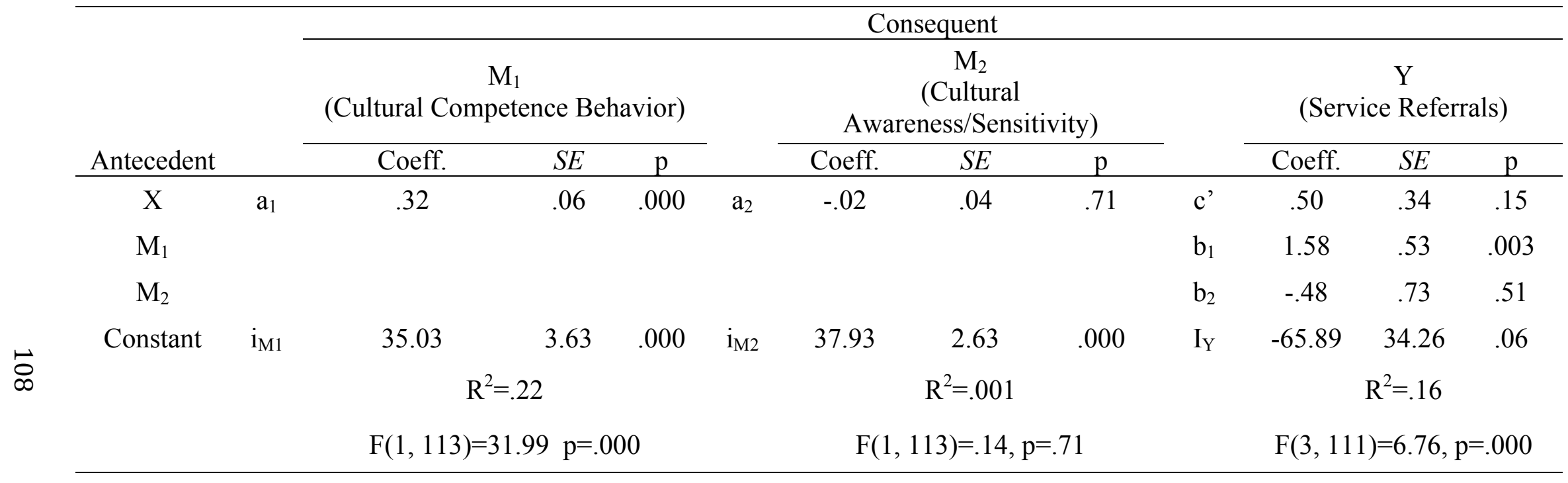


Figure 4-4 indicates the cultural competence (cultural competence behavior and cultural awareness/sensitivity) mediation effect on the association between service providers' familiarity with community resources and service referrals by domains.

In all six domains, cultural $\mathrm{CCB}$ is well explained by familiarity with community resources. In contrast, the indirect effects of familiarity with community resources on all domains' service referrals through CAS is not statistically different from zero. Thus, the effect of the familiarity with community resources on all service referrals was not mediated by CAS.

More specifically, for orientation service referrals ( $95 \%$ confidence interval is from .01 to .04), basic needs service referrals ( $95 \%$ confidence interval is from .02 to .11), assistance to access benefits service referrals ( $95 \%$ confidence interval is from .01 to .06), employment service referrals ( $95 \%$ confidence interval is from .001 to .03), health service referrals ( $95 \%$ confidence interval is from .002 to .05), and other service referrals ( $95 \%$ confidence interval is from .001 to .04 ) were significantly mediated by familiarity with community resources through CCB. CI (95\%) not including zero indicates significant indirect effects and, thus, that the effect of the familiarity with community resources on all service referrals was mediated by the cultural competence behavior. 


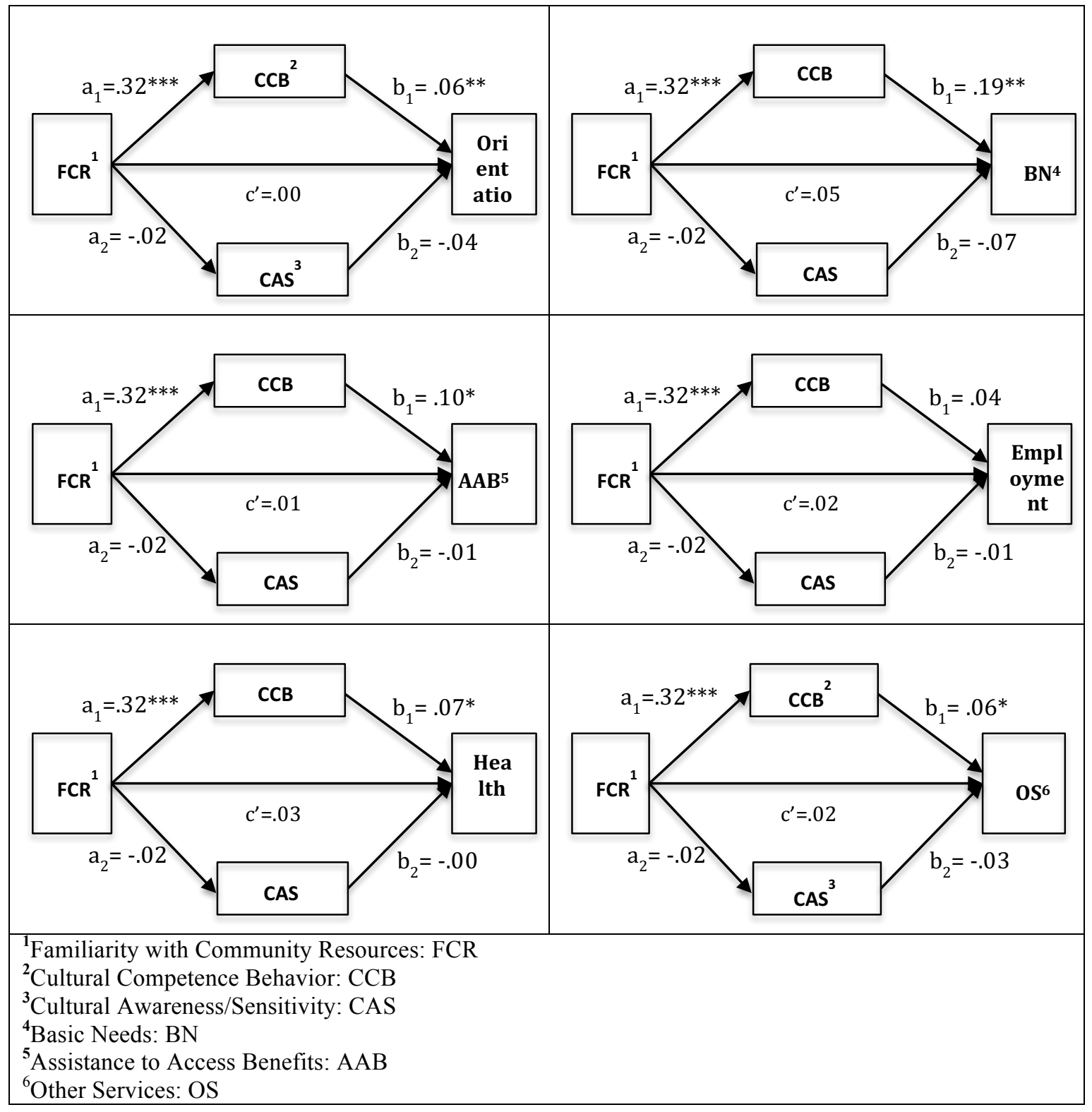

Figure 4-4 Cultural Competence Mediate Association between Familiarity with Community Resources and Service Referrals by Domains

Figure 4-5 and Table 4-21 explain the overall cultural competence (cultural competence behavior and cultural awareness/sensitivity) mediation effect on the association between service providers' work experience and service provision. 
CAS is explained by the work year $\left(\mathrm{R}^{2}=.005\right)$, which is not significant; cultural CCB is well explained by work experience $\left(\mathrm{R}^{2}=.14\right)$. More specifically, the indirect effect of work experience on service provision through $\mathrm{CCB}$ is the product of the effect of work experience on $\mathrm{CCB}$ ( $\mathrm{a}_{1}$ from figure) and the effect of $\mathrm{CCB}$ on service provision ( $\mathrm{b}_{1}$ from figure), holding all else constant. That is, $\mathrm{a}_{1} \mathrm{~b}_{1}=(.30) *(2.10)=.63$. So, work experience seems to increase service provision by .63 units, indirectly, through its effect on increasing CCB, which in turn leads to higher service provision. The $95 \%$ confidence interval is from .08 to 1.56 . CI (95\%) not including zero indicates significant indirect effects and, thus, that the effect of the work experience on service provision is mediated by the cultural competence behavior.

The indirect effects of work experience on service provision through CAS is not statistically different from zero ( $95 \%$ confidence interval from -.10 to .61$)$. Thus, the effect of the work experience on service provision was not mediated by CAS.

The direct effect of work experience, $c^{\prime}=.95$, is the estimated difference in service provision between two service providers experiencing the same level of cultural competence behavior but who differ by one unit in their reported work experience. However, this direct effect is not statistically different from zero, $\mathrm{t}(111)=1.07, \mathrm{p}=.29$, with a $95 \%$ confidence interval from -.80 to 2.70 .

Observe that the total effect $\mathrm{C}$ is the sum of the direct effect of work experience and the two indirect effects of work experience, through $\mathrm{CCB}$ and CAS. That is, $\mathrm{C}=\mathrm{c}$ ' + $\mathrm{a}_{1} \mathrm{~b}_{1}+\mathrm{a}_{2} \mathrm{~b}_{2}=(.95)+(.63)+(.10)=1.68$. However, this total effect is not statistically different from zero ( $95 \%$ confidence interval from -.12 to 3.47$)$. 


\section{$\mathrm{X}$ : Work Experience}

Y: Service Provision

$\mathrm{M}_{1}$ : Cultural Competence Behavior

$\mathrm{M}_{2}$ : Cultural Awareness/Sensitivity

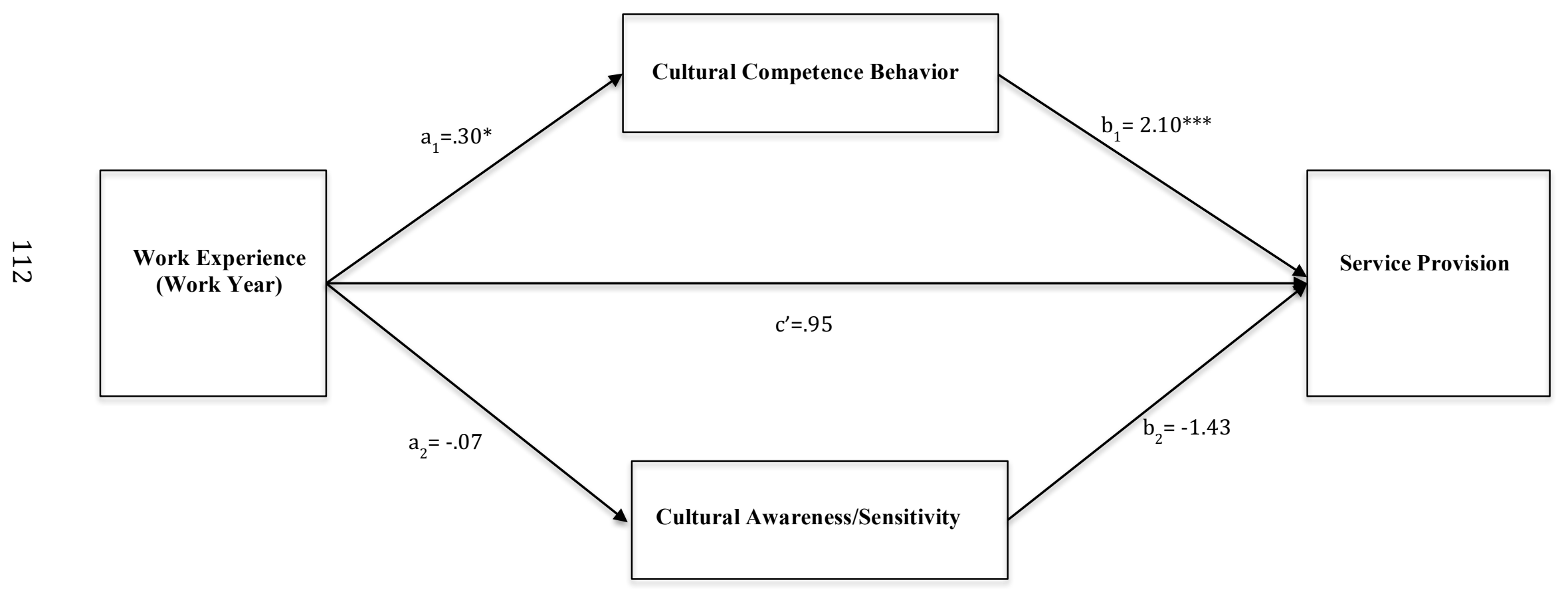

Figure 4-5 Parallel Multiple Mediator Model 3 
Table 4-21 Regression Coefficients, Standards Errors, and Model Summary Information for the Parallel Multiple Mediator Model Depicted in Figure 4-3.

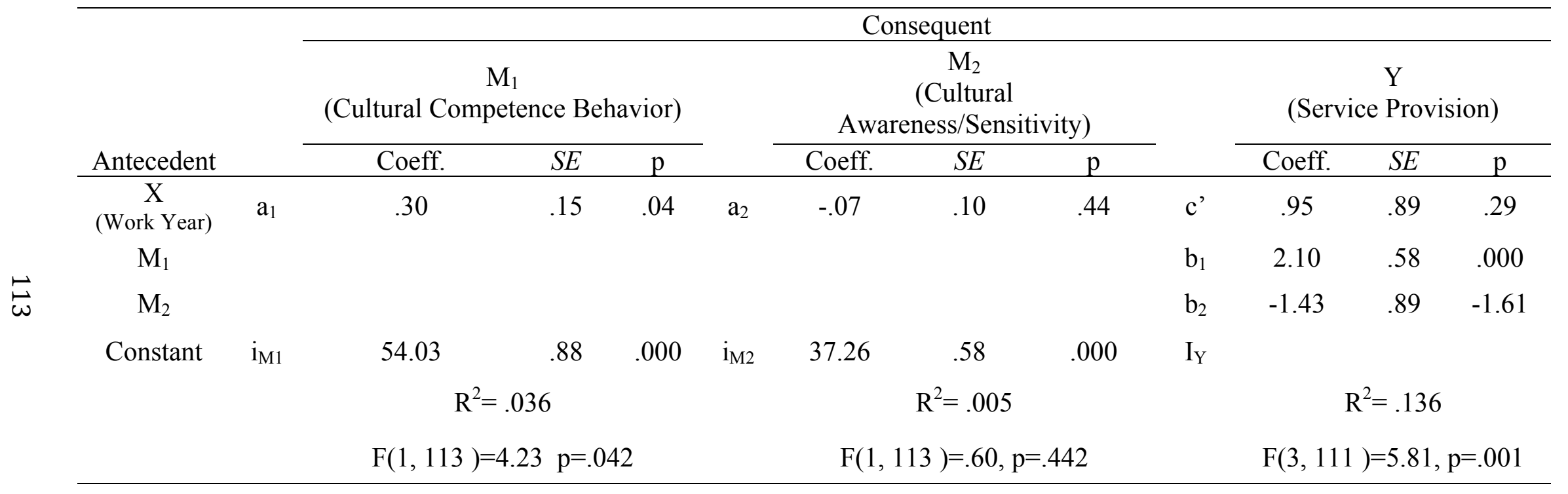


Figure 4-6 indicates the cultural competence (cultural competence behavior and cultural awareness/sensitivity) mediation effect on the association between service providers' work experience and service provision by domains. Four service provision domains were found to be indirect effects of work experience through CCB: orientation, basic needs, assistance to access benefits, and other service provision.

More specifically, orientation service provision ( $95 \%$ confidence interval is from .001 to .05), basic needs service provision ( $95 \%$ confidence interval is from .01 to .20 ), assistance to access benefits service provision ( $95 \%$ confidence interval is from .004 to .10), and other service provision (95\% confidence interval is from .001 to .07) were significantly mediated by work experience through CCB. CI (95\%) not including zero indicates significant indirect effects and, thus, that the effect of the work experience on four service referrals was mediated by the cultural competence behavior. On the other hand, the indirect effects of work experience on four service referrals through CAS were not statistically different from zero. Thus, the effect of work experience on indicated service referrals was not mediated by CAS. 


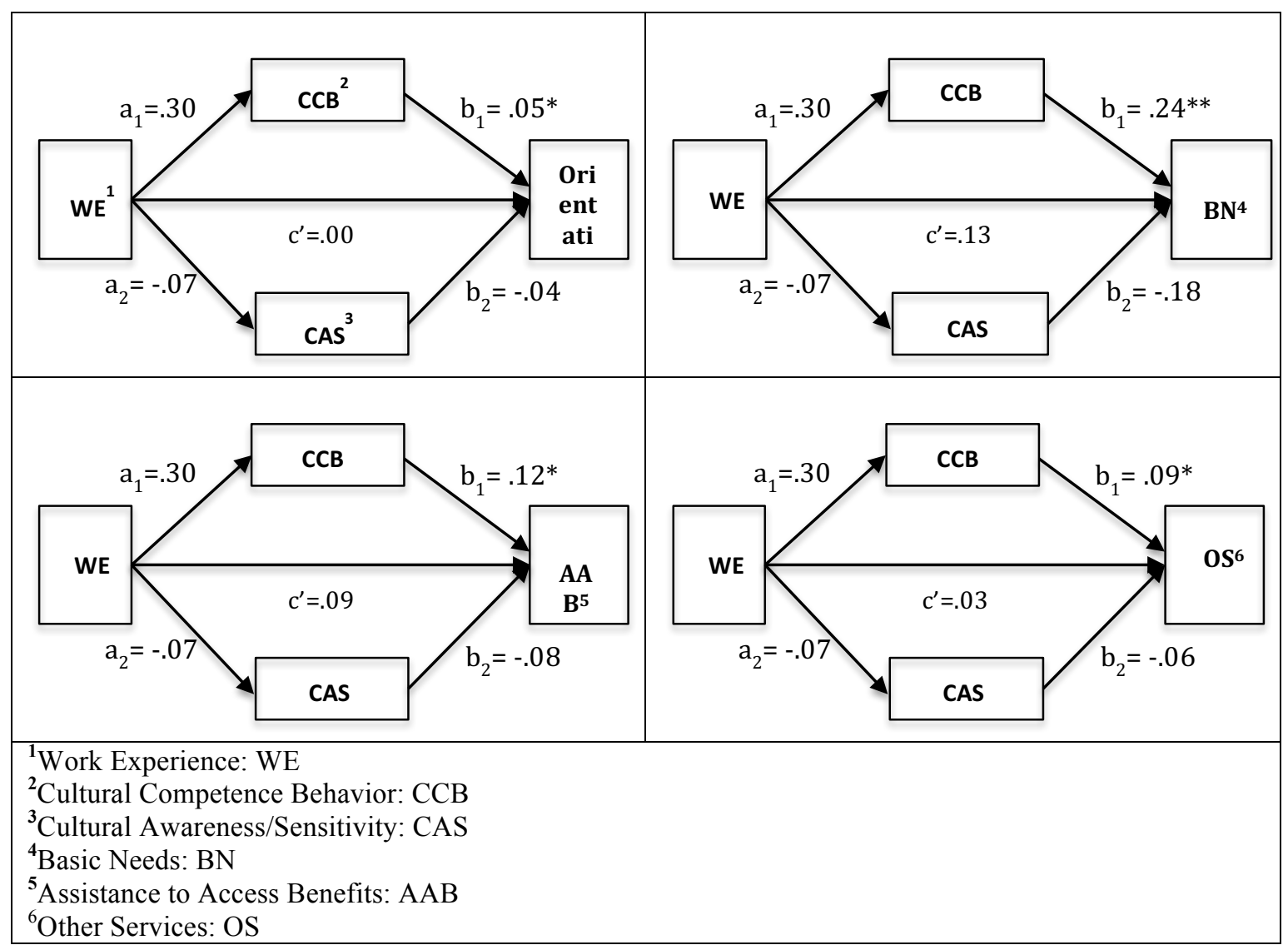

Figure 4-6 Cultural Competence Mediate Association between Work Experience and Service Provision by Domains

Figure 4-7 and Table 4-22 indicate the overall cultural competence (cultural competence behavior and cultural awareness/sensitivity) mediation effect on the association between service providers' work experience and service referrals.

CAS is explained by the work experience $\left(\mathrm{R}^{2}=.01\right)$, which is not significant; cultural CCB is well explained by work experience $\left(\mathrm{R}^{2}=.15\right)$. More specifically, the indirect effect of work experience on service referrals through $\mathrm{CCB}$ is the product of the effect of work experience on CCB ( $a_{1}$ from figure) and the effect of CCB on service referrals $\left(b_{1}\right.$ from figure), holding all else constant. That is, $a_{1} b_{1}=(.30) *(1.85)=.56$. So, work experience seems to increase service referrals by .56 units, indirectly, through its 
effect on increasing CCB, which in turn leads to higher service referrals. The $95 \%$ confidence interval is from .02 to 1.36 . CI (95\%) not including zero indicates significant indirect effects and, thus, that the effect of the work experience on service referrals is mediated by the cultural competence behavior.

The indirect effects of work experience on service referrals through CAS is not statistically different from zero (95\% confidence interval from -.03 to .19$)$. Thus, the effect of the work experience on service referrals was not mediated by CAS.

The direct effect of work experience, $c^{\prime}=.74$, is the estimated difference in service referrals between two service providers experiencing the same level of cultural competence behavior but who differ by one unit in their reported work experience. However, this direct effect is not statistically different from zero, $t(111)=1.79, p=.08$, with a $95 \%$ confidence interval from -.14 to 2.81 .

Observe that the total effect $\mathrm{C}$ is the sum of the direct effect of work experience and the two indirect effects of work experience, through $\mathrm{CCB}$ and CAS. That is, $\mathrm{C}=\mathrm{c}$ ' + $a_{1} b_{1}+a_{2} b_{2}=(.74)+(.56)+(.04)=1.34$. However, this total effect is not statistically different from zero ( $95 \%$ confidence interval from -.69 to 2.17$)$. 
$\mathrm{X}$ : Work Experience

Y: Service Referrals

$\mathrm{M}_{1}$ : Cultural Competence Behavior

$\mathrm{M}_{2}$ : Cultural Awareness/Sensitivity

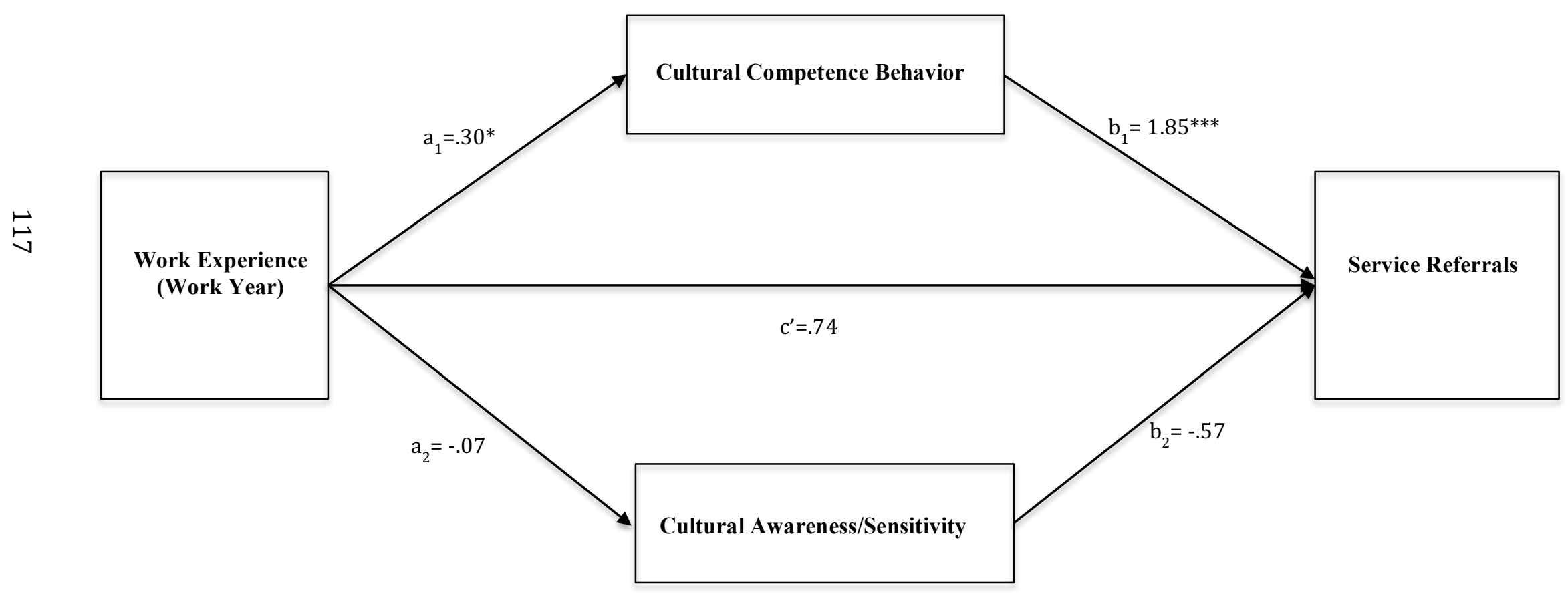

Figure 4-7 Parallel Multiple Mediator Model 4. 
Table 4-22 Regression Coefficients, Standards Errors, and Model Summary Information for the Parallel Multiple Mediator Model Depicted in Figure 4-4.

\begin{tabular}{|c|c|c|c|c|c|c|c|c|c|c|c|c|}
\hline \multirow{2}{*}{ Antecedent } & & \multicolumn{11}{|c|}{ Consequent } \\
\hline & & \multicolumn{3}{|c|}{$\begin{array}{c}\mathrm{M}_{1} \\
\text { (Cultural Competence Behavior) }\end{array}$} & & \multicolumn{3}{|c|}{$\begin{array}{c}\mathrm{M}_{2} \\
\text { (Cultural } \\
\text { Awareness/Sensitivity) }\end{array}$} & & \multicolumn{3}{|c|}{$\begin{array}{c}\text { Y } \\
\text { (Service Referrals) }\end{array}$} \\
\hline $\begin{array}{c}\mathrm{X} \\
\text { (Work Year) }\end{array}$ & $\mathrm{a}_{1}$ & .30 & .15 & .042 & $a_{2}$ & -.07 & .10 & .44 & $c^{\prime}$ & .74 & .72 & .31 \\
\hline \multicolumn{2}{|l|}{$\mathrm{M}_{1}$} & & & & & & & & $\mathrm{~b}_{1}$ & 1.85 & .47 & .000 \\
\hline \multirow[t]{3}{*}{ Constant } & $\mathrm{i}_{\mathrm{M} 1}$ & 54.03 & .88 & .000 & $\mathrm{i}_{\mathrm{M} 2}$ & 37.26 & .58 & .000 & $\mathrm{I}_{\mathrm{Y}}$ & -48.81 & 32.12 & .13 \\
\hline & & \multicolumn{3}{|c|}{$\mathrm{R}^{2}=.036$} & & \multicolumn{3}{|c|}{$\mathrm{R}^{2}=.005$} & & \multicolumn{3}{|c|}{$\mathrm{R}^{2}=.147$} \\
\hline & & \multicolumn{3}{|c|}{$\mathrm{F}(1,113)=4.23, \mathrm{p}=.042$} & & \multicolumn{3}{|c|}{$F(1,113)=.60, p=.442$} & & \multicolumn{3}{|c|}{$\mathrm{F}(3,111)=6.36, \mathrm{p}=.001$} \\
\hline
\end{tabular}


Figure 4-8 presents the cultural competence (cultural competence behavior and cultural awareness/sensitivity) mediation effect on the association between service providers' work experience and service referrals by domains. Five service referral domains were found to have indirect effects of work experience through CCB: orientation, basic needs, assistance to access benefits, employment, and other service referrals. On the other hand, none of the indirect effects of work experience on service referral domains through CAS were founded. Thus, the effect of work experience on each domain of service referral was not mediated by CAS.

Detailed mediation effects of each domain, orientation service referrals $(95 \%$ confidence interval is from .001 to .05), basic needs service referrals ( $95 \%$ confidence interval is from .004 to .15), assistance to access benefits service referrals ( $95 \%$ confidence interval is from .003 to .08), employment service referrals ( $95 \%$ confidence interval is from .002 to .05$)$, and other service referrals $(95 \%$ confidence interval is from .001 to .06) were significantly mediated by work experience through CCB. CI (95\%) not including zero indicates significant indirect effects and, thus, that the effect of the work experience on four service referrals was mediated by the cultural competence behavior. 


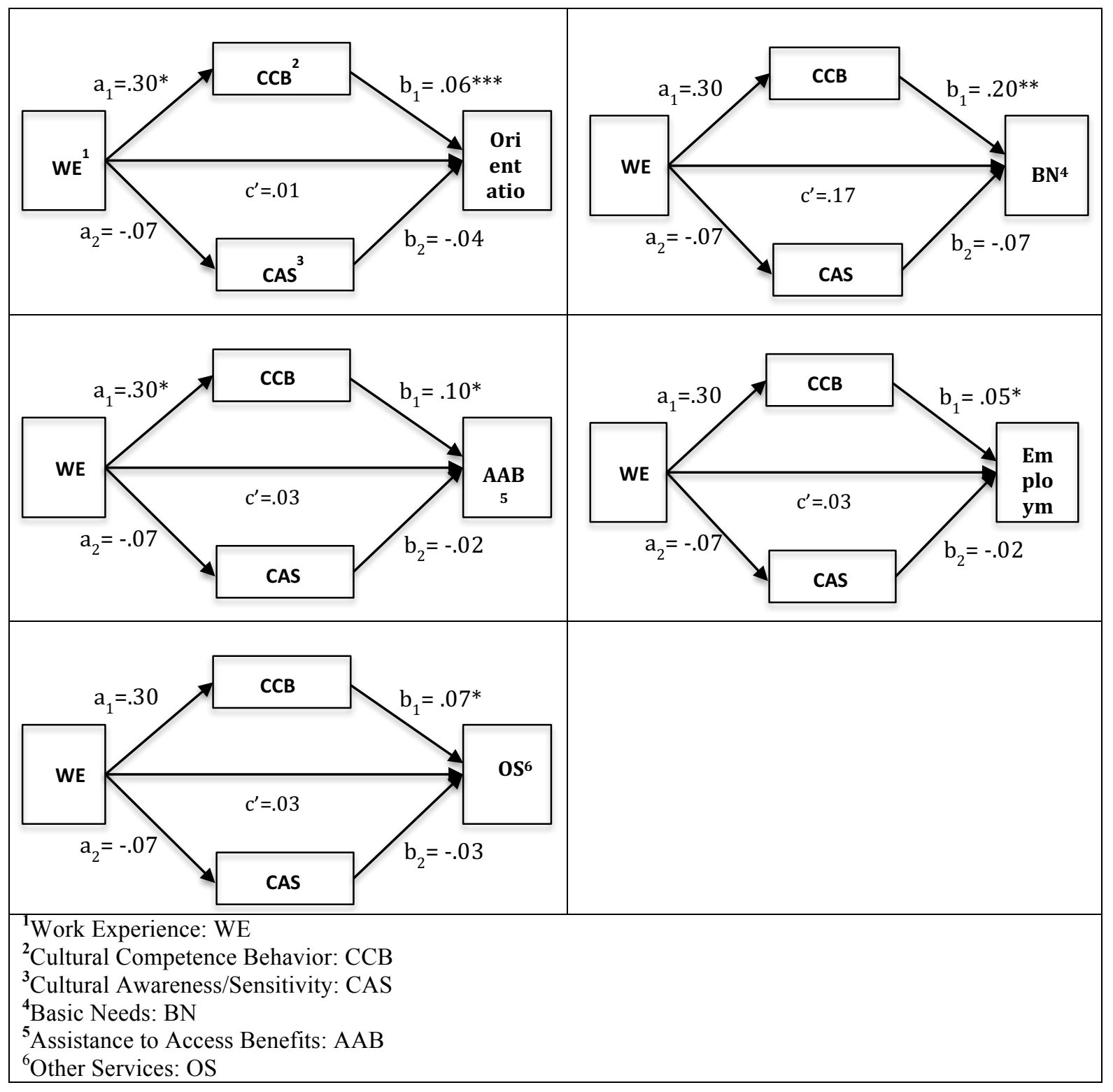

Figure 4-8 Cultural Competence Mediate Association between Work Experience and Service Referrals by Domains

\subsection{Summary}

The purpose of this research was to examine the role of refugee service providers' personal and organizational factors in providing service to refugees. Relationships among 
selected concepts from the gateway provider model, cultural competence model, and organization theory measured by an online survey and characteristics of refugee service providers were investigated and are reported here. Univariate, bivariate, correlation, multiple regression, and mediation data analyses were conducted to answer the research questions.

Univariate and bivariate analyses presented the general characteristics of all concepts involved in this research. Correlation indicates that all concepts investigated are significantly correlated at the $\mathrm{p}<.001$ level. Multiple regression revealed that the personal factors (knowledge of refugees, familiarity with community resources, and cultural competence) and organizational culture factors (proficiency, rigidity, and resistance) are related to each domain of service provision and referral. In addition, it provided a statistically significant model for the prediction of service provision and referrals to refugees. All models of domains of service provision and five models of domains of service referral were statistically significant.

Finally, a mediation analysis indicated mediation effects of cultural competence on the association between service providers' familiarity with community resources and service provision and referrals. Moreover, cultural competence's mediation effect was founded on associations between work experiences and service provision and referrals.

Chapter 5 includes the interpretation of the findings, limitations of the study, recommendations, implications, and conclusions. 


\section{CHAPTER 5: DISCUSSION AND IMPLICATIONS}

The purpose of this study was to investigate the relationships among the following variables: service provision, service referral, personal factors (knowledge of refugee, familiarity with community resources, and cultural competence), and organizational factors (proficiency, rigidity, and resistance). This study was conducted because insights into this relationship may assist refugee service providers when they provide service to their clients. Therefore, this study examined the importance of the roles of service providers who provide service to refugee clients. It was hypothesized that there are direct association between personal factors and service provision/referral for refugees.

Similarly, it was hypothesized that there are direct associations between organizational culture factors and service provision/referral for refugees. Lastly, it was hypothesized that cultural competence would mediate the relationship between familiarity with community resources and service provision/referral for refugees and would mediate between work experience and service provision/referral for refugees. Some hypotheses were supported.

The key findings of this study are that knowledge of refugee, familiarity with community resources, and cultural competence were significantly associated with service provision/referral when conducting the multiple regression analysis. For mediation analysis, cultural competence behavior significantly mediated the relationship between familiarity with community resources and service provision/referral and between work experience and service provision/referral. These findings can provide helpful information related to refugee service providers, refugee service organizations, and the refugees.

This chapter first address the hypotheses and reasons for which they were not 
supported. Next is an interpretation of this study's major findings and an explanation of how those findings relate to the literature. Then, implications based on major findings are discussed. Lastly, study limitations are addressed and recommendations are made for future research.

\subsection{Discussion of Hypotheses}

This study has five research questions based upon the three aims of the study. Following are those research questions: 1) What type of services are currently being provided to meet the meet the need of refugees? 2-1) What are the relative effects of personal factors (knowledge of refugees, familiarity with community resources, and cultural competence) and organizational culture factors (proficiency, rigidity, and resistance) on service provision for refugees? 2-2) What are the relative effects of personal factors (knowledge of refugees, familiarity with community resources, and cultural competence) and organizational culture factors (proficiency, rigidity, and resistance) on service referrals for refugees? 3) Does cultural competence behavior and cultural awareness/sensitivity mediate the associations between familiarity with community resources and service provision or referrals for refugees? 4) Does cultural competence behavior and cultural awareness/sensitivity mediate the associations between work experience and service provision or referrals for refugees? In order to answer those research questions and associated hypotheses, data analysis was conducted and the results were explored in the previous section. This section answers research question 1 and the findings and discusses whether or not the hypotheses were supported. 


\section{Research Question 1: What type of services are currently being provided to}

meet the needs of refugees? In order to answer research question 1, the number of service provision and referrals were calculated to rank the type of services provided or referred. As Table 4-21 indicates, for service provision, most frequently provided services by refugee service provider were case management, followed by transportation support, cultural orientation, and translation support. On the other hand, providing religious information service provision was the least provided service. Next, for service referral, health insurance support and health screening support were the most frequently referred to other agencies by refugee service providers, followed by medical appointment and ESL class enrollment support. On the contrary, airport reception service was the least frequently referred to other agencies, followed by health security orientation service and providing religious information service referral. 
Table 5-1 Service Ranking of Most Frequently Provided and Referred

(A higher score indicates a higher degree of service provision and referrals)

\begin{tabular}{|c|c|c|c|c|c|}
\hline \multicolumn{3}{|c|}{ Service Provision } & \multicolumn{3}{|c|}{ Service Referral } \\
\hline Rank & Service & Number & Rank & Service & Number \\
\hline 1 & Case management & 479 & 1 & Health insurance support & 238 \\
\hline 2 & Transportation support & 457 & 2 & Health screening support & 232 \\
\hline 3 & Cultural orientation & 392 & 3 & Medical appointment support & 229 \\
\hline 4 & Translation support & 388 & 4 & ESL Class enrollment support & 228 \\
\hline 5 & Providing household supplies & 362 & 5 & Employment support & 227 \\
\hline 6 & Employment support & 350 & 6 & Immigration legal support & 221 \\
\hline 6 & Health safety orientation & 350 & 7 & Food Stamp (SNAP) & 211 \\
\hline 8 & Medical support & 341 & 7 & Financial support & 211 \\
\hline 9 & Providing clothes & 336 & 9 & Providing food & 209 \\
\hline 10 & ESL Class enrollment support & 335 & 10 & Providing clothes & 198 \\
\hline 11 & Providing food & 324 & 11 & Job training & 188 \\
\hline 12 & Job Training & 322 & 12 & Case management & 186 \\
\hline 13 & Housing support & 320 & 13 & Transportation support & 180 \\
\hline 14 & Providing furniture & 317 & 13 & School enrollment support & 180 \\
\hline 15 & Financial support & 311 & 15 & Translation support & 165 \\
\hline 16 & School enrollment support & 305 & 16 & Social security support & 146 \\
\hline 17 & Social security support & 300 & 17 & Providing furniture & 144 \\
\hline 18 & Airport reception & 293 & 18 & Housing support & 138 \\
\hline 19 & Health insurance support & 285 & 19 & Cultural orientation & 129 \\
\hline 20 & Food Stamp (SNAP) & 278 & 20 & Providing household supplies & 127 \\
\hline 21 & Health screening support & 216 & 21 & Health security orientation & 114 \\
\hline 22 & Immigration legal support & 211 & 21 & Providing religious info. & 114 \\
\hline 23 & Providing religious info. & 46 & 23 & Airport reception & 65 \\
\hline
\end{tabular}

\section{Research Hypothesis 2-1-1: Personal factors are stronger than organizational}

culture factors in predicting service provision for refugees. Hypothesis 2-1-1 was

supported. Personal factors, such as knowledge of refugees, familiarity with community

resources, and cultural awareness/sensitivity had strong associations with service

provision compared to organizational culture factors, such as proficiency culture.

More specifically, knowledge of refugee $(\beta=.17, p \leq .10)$ was significantly

associated with orientation service provision (Domain 1). This means that refugee service 
providers who had more knowledge of refugee issues provided more orientation service to refugees than those who did not.

The familiarity with community resources was significantly associated with most of the service provision except health service provision (Domain 5). Service providers' familiarity with community resources positively associated with their service provision. Refugee service providers who had a higher level of familiarity with community resources provided more orientation service $(\beta=.18, p \leq .10)$, basic needs service ( $\beta$ $=.29, p \leq .01)$, assistance to access benefits service $(\beta=.27, p \leq .01)$, employment service $(\beta=.25, p \leq .05)$, and other service $(\beta=.27, p \leq .01)$ to refugees than those who did not.

Conversely, service providers' cultural awareness/sensitivity was negatively associated with health service provision $(\beta=-.25, p \leq .05)$. This indicates that service providers who had a higher degree of cultural awareness/sensitivity provided health service to refugees less often.

For organizational culture factors, proficiency culture was the only factor entered into the regression model. However, proficiency was positively associated with only employment service provision (Domain $4 ; \beta=.19, p \leq .05$ ). Refugee service providers who were working in a proficient cultural organization provided greater employment service to refugees than those who did not work in that type of organization. Skilled and experienced organizational culture employers helped service providers to provide employment service to refugees.

Research Hypothesis 2-2-1: Personal factors are stronger than organizational culture factors in predicting service referral for refugees. Hypothesis 2-2-1 was 
partially supported. Personal factors, particularly cultural competence behavior, had a strong association with service referral compared to organizational culture factors, such as proficiency, rigidity, and resistance culture.

More specifically, cultural competence behavior was significantly associated with all referral subdomains but Domain 5. This means that service providers' cultural competence behavior positively related to their service referral. Refugee service providers who had a higher level of cultural competence behavior referred more orientation service $(\beta=.32, p \leq .01)$, basic needs service $(\beta=.34, p \leq .001)$, assistance to access benefits service $(\beta=.32, p \leq .01)$, employment service $(\beta=.26, p \leq .05)$, and other service $(\beta=.28, p \leq .01)$ to refugees than those who did not.

For organizational culture factors, rigidity culture was negatively associated with assistance to access benefits service referral (Domain $3 ; \beta=.23, p \leq .10$ ) and employment service referral (Domain $4 ; \beta=.23, p \leq .10$ ). Refugee service providers who were working in a rigidity culture organization provided assistance to access benefit and employment referral service to refugees less than those who did not.

\section{Research Hypothesis 3-1: Cultural competence behavior will positively} mediate the association between familiarity with community resources and service provision for refugees. Hypothesis 3-1 was supported. Cultural competence behavior did mediate the relationship between service providers' familiarity with community resources and service provision. The level of familiarity with community resources seemed to related to service provision indirectly through its effect on increasing cultural competence behavior (95\% confidence interval from .17 to 1.08); although, a direct effect between service provider's familiarity with community resources and overall service provision 
was not statistically different from zero, $\mathrm{t}(111)=.72, \mathrm{p}=.09$, with a $95 \%$ confidence interval from -.11 to 1.55 . That indicates that service providers' level of cultural competence behavior plays a significant role when service providers provide service to refugee clients.

Each subdomain for cultural competence mediated the association between familiarity with community resources and service provision. Three service provision domains had significant mediation effect with 95\% confidence: basic needs (Domain $2 ; .01-.12$ ), assistance to access benefits (Domain $3 ; .00-.06$ ), and other services (Domain $6 ; .00-.04)$

\section{Research Hypothesis 3-2: Cultural competence behavior will positively} mediate the association between familiarity with community resources and service referral for refugees. Hypothesis 3-2 was supported. Cultural competence behavior did mediate the relationship between service providers' familiarity with community resources and service referral. The level of familiarity with community resources seemed to increase service referral indirectly through its effect on increasing cultural competence behavior (95\% confidence interval from .22 to .93$)$; although, a direct effect between service provider's familiarity with community resources and overall service referral was not statistically different from zero, $\mathrm{t}(111)=1.44, \mathrm{p}=.15$, with a $95 \%$ confidence interval from -.18 to 1.18. That indicates an important role of cultural competence behavior of refugee service providers.

Each subdomain for cultural competence behavior mediated the association between familiarity with community resources and service referral. All six service referral domains had significant mediation effect with $95 \%$ confidence: orientation 
(Domain 1; .01-.04), basic needs (Domain 2;.02-.11), assistance to access benefits (Domain 3;.01-.06), employment (Domain 4; .00-.03), health (Domain 5;.00-.05), and other services (Domain 6; .00-.04) service referral.

\section{Research Hypothesis 3-3: Cultural awareness/sensitivity will positively} mediate the association between familiarity with community resources and service provision for refugees. Hypothesis 3-3 was not supported. Both the overall mediation model and each subdomain of cultural awareness/sensitivity had effects on the relationship between service providers' familiarity with community resources and service provision that were not significantly different from zero.

\section{Research Hypothesis 3-4: Cultural awareness/sensitivity will positively} mediate the association between familiarity with community resources and service referral for refugees. Hypothesis 3-4 was not supported. Both the overall mediation model and each subdomain of cultural awareness/sensitivity had effects on the relationship between service providers' familiarity with community resources and service referral that were not significantly different from zero.

\section{Research Hypothesis 4-1: Cultural competence behavior will positively} mediate the association between work experience and service provision for refugees. Hypothesis 4-1 was supported. Cultural competence behavior did mediate the relationship between service providers' work experience and service provision. Level of work experience seemed to increase service provision indirectly through its effect on increasing cultural competence behavior ( $95 \%$ confidence interval from .08 to 1.56); although, the direct effect between service provider's work experience and overall service provision was not statistically different from zero, $\mathrm{t}(111)=1.07, \mathrm{p}=.29$, with a $95 \%$ 
confidence interval from -.80 to 2.70 . This means that service providers' work experience could not explain service provision without service providers' cultural competence behavior.

Each subdomain for cultural competence behavior mediated the association between work experience and service provision. Four service provision domains had a significant mediation effect with 95\% confidence: orientation (Domain 1;.00-.05), basic needs (Domain $2 ; .01-.20$ ), assistance to access benefits (Domain $3 ; .00-.10$ ), and other services (Domain 6;.00-.07) service provision.

\section{Research Hypothesis 4-2: Cultural competence behavior will positively} mediate the association between work experience and service referral for refugees.

Hypothesis 4-2 was supported. Cultural competence behavior did mediate the relationship between service providers' work experience and service referral. The level of work experience seemed to increase service referral indirectly through its effect on increasing cultural competence behavior ( $95 \%$ confidence interval from .02 to 1.36); although, the direct effect between service provider's work experience and overall service referral was not statistically different from zero, $\mathrm{t}(111)=1.79, \mathrm{p}=.08$, with a $95 \%$ confidence interval from -..14 to 2.81 . This indicates an important role of service providers' cultural competence behavior when providing referral service to refugee clients.

Each subdomain for cultural competence behavior mediated an association between work experience and service referral. Five service referral domains had significant mediation effect with $95 \%$ confidence: orientation (Domain $1 ; .00-.05$ ), basic 
needs (Domain 2;.00-.15), assistance to access benefits (Domain 3; .00-.08),

employment (Domain 4; .00-.05), and other services (Domain 6;.00-.06) service referral.

\section{Research Hypothesis 4-3: Cultural awareness/sensitivity will positively}

mediate the association between work experience and service provision for refugees.

Hypothesis 4-3 was not supported. Both the overall mediation model and each subdomain

of cultural awareness/sensitivity had effects on the relationship between service

providers' work experience and service provision that were not significantly different

from zero.

Research Hypothesis 4-4: Cultural awareness/sensitivity will positively mediate the association between work experience and service referral for refugees.

Hypothesis 4-4 was not supported. Both the overall mediation model and each subdomain of cultural awareness/sensitivity had effects on the relationship between service providers' work experience and service referral that were not significantly different from zero.

\subsection{Interpretation of the Major Findings}

An interpretation of major findings is presented by variables. The demographic variable includes race/ethnicity, education, and job title. Knowledge of refugee, familiarity with community resources, and cultural competence are explained in personal factors. In addition, proficiency and rigid organizational culture are mentioned in organizational factor. 


\subsubsection{Demographics}

There were two variables of demographics that had a significant relationship with overall service provision and referral: race/ethnicity and job title. However, in the regression results of each domain, the level of education was associated with the subdomain of basic needs and employment service provision.

Race/Ethnicity. Service providers' race/ ethnicity had a positive relationship with orientation service provision in contrast to a negative relationship with basic needs, assistant to access benefits, and other services referral. In other words, white service providers provided more orientation service to refugees than non-white service providers. In the service referral provision, non-white service providers (i.e., Native American or American Indian, Hispanic or Latino, Asian, and Black or African American) provided more referral service than white refugee service providers.

Education Level. Service providers' education level was negatively associated with basic needs and employment service provision. Service providers who had a higher education level (graduate and above) were unlikely to provide basic needs and employment service to refugees than those who had a lower level of education. There is no exact reason as to why those two domains had a detected association with education. However, it is assumed that in the refugee service provision setting, the ability of service provision might be less influenced by educational level. In other words, service providers' field experience or awareness of refugee needs may affect service provision to refugees more.

Job Title. Service providers' job title was only associated with service provided. Service providers who had a resettlement related job title (i.e., resettlement specialist, 
case manager, social worker, and program manager) provided more service than other job titles, such as employment, health, and education related job titles. These results might be due to the refugees' many needs related to resettlement and adjusting to a new place. Most of those services are provided at an agency focused on refugee resettlement. Therefore, service providers with a resettlement related job title could have more chances to provide service to refugees than those with other titles.

\subsubsection{Personal Factors}

Knowledge of Refugee. Data from this study revealed knowledge of refugee has a significant relationship with orientation service provision (Domain 1). This means that providers who are more knowledgeable about refugee issues provide more orientation service to refugees. At most of the refugee agencies, service providers who can speak a newcomer's language provide orientation service, such as cultural and health orientation, to refugees. Cultural orientation generally takes place the first day or at least by the second day that the refugee arrives in the U.S. The provider explains about the different culture and regulations, and the attitude the new refugee needs in order to resettle and adjust to a new life in the U.S. (Cultural Orientation Resource Center, n.d.). Therefore, there is little wonder that a service provider who speaks the same language will provide orientation service and that they may have more knowledge about refugee issues than others.

No past study has examined the relationship between service providers' knowledge of refugee and refugee service provision, especially orientation service, but there has been research about other professions' knowledge of their clients. In Yi, Kim, Kim, and Hong's (2016) study, social service providers who provide service to 
immigrants identified a lack of awareness of their client's issues and that it was a major barrier to effective services. In addition, Onoka, Onwujekwe, Hanson, \& Uzochukwu (2012) conducted a study on the relationship between service provider factors and service provision for individuals with intermittent preventive treatment for malaria in pregnancy (IPTp). They found that knowledge of IPTp was the most significant predictor for effective service provision. The findings that knowledge of clients affects service provision is similar to results from previous studies.

Familiarity with Community Resources. The second key finding of personal factor is that there was a significant relationship between refugee service providers' familiarity with community resources and service provision: orientation, basic needs, assistance to access benefits, employment, and other services. Service providers who are familiar with community resources, such as school information, job information, and general public services, have more chance to provide service to refugees. A previous study by Stiffman et al. (2000) had similar findings. According to their study, child service providers who had greater resource knowledge was associated with increased service provision. Moreover, according to Yi, Kim, Kim, and Hong's (2016) study, in their qualitative interview, they found the biggest challenge experienced by social service providers working with immigrant adolescents was a lack of knowledge of resources and collaborations. Tastsoglou, Abidi, Brigham, and Lange (2014) also presented a similar result in a qualitative study of service providers in relation to women refugee claimants in Atlantic Canada. They found that services and resources to support refugees were significant factors on navigating services.

Refugees are confronted with various problems, such as mental health issues, 
poverty, isolation, language barriers, and employment. Therefore, having a familiarity and knowledge about many community or social services is essential.

Cultural Competence. Two variables were measured to explain service providers' cultural competence: cultural competence behavior and cultural awareness/sensitivity.

First, cultural competence behavior was identified as a critical predictor of overall and each domains of service referral only. Service providers who had a higher degree of culturally competent behavior referred more services than those who did not. Service referral could be a more active type of service provision than service provided. This is because services which service providers provide to refugees may already be decided by the organization. For example, resettlement agencies are responsible for providing airport reception, housing service, and cultural orientation to new arrival refugees. Therefore, regardless of the service provider's special effort, service could be provided. However, the service provider's awareness of refugee needs may lead the provider to make extra efforts to look for other agencies that can provide culturally appropriate service to the refugee. Therefore, a high level of cultural competence behavior has a significant association with service referrals.

The importance of cultural competence behavior was also shown as a result of mediation analysis. Cultural competence behavior played a crucial role in the association between familiarity with community resources and work experience and service provision/referral. There was no direct relationship between two predictors and service provision and referral, but adding cultural competence behavior as a mediator, the indirect effect was detected. It clearly showed that service providers' cultural competence 
level was a powerful predictor of explaining refugee service provision. In addition, it can be useful evidence to develop cultural competence training, especially for refugee service providers.

On the other hand, an interesting finding was that cultural awareness/sensitivity (CAS) was not identified as a critical predictor of overall service provision model. However, in the regression result of each domain, CAS was negatively associated with health service provision. It indicated that a high level of service provider's culture awareness/sensitivity might not affect action (service provision). In other words, service providers who are far too sensitive of culture might not provide service all the more. It was especially negatively associated with health service provision. The health service provision domain includes medical appointment support, health screening, and health insurance support. Health issues may be more sensitive and have more culture barriers (Håkonsen, Lees, \& Toverud, 2014), particularly in the use of medicine and language, than other services. For this reason, service providers might be aware that some kind of cultural competence health service provision is needed. This being so, they might decide not to provide any service rather than make mistakes during the service provision procedure. Findings in this study were consistent with previous research that studied cultural competence of public health nurses (PHNs) using cultural awareness and sensitivity, cultural competence behavior, and diversity experience (Starr \& Wallace, 2009). They found that nurses have moderate competence in thought (awareness and sensitivity), but this did not show in a comparable level of behaviors (cultural competence service provision). In addition, according to Wilbur's (2008) study of factors that influence the cultural competence of nurse practitioner students in the U.S., they 
found that being culturally aware is essential but not sufficient to be culturally competent nurse practitioners. It looked like cultural awareness is the first step in preparing to provide culturally competent services (Hart \& Mareno, 2016). Therefore, this can be useful information for the refugee service providers as well as agencies, as it can be applied to emphasize the importance of cultural competence training. This training might help service providers move to the next step, such as convert service providers' cultural awareness/sensitivity into action. More detailed information of cultural competence training was discussed in the implication part.

\subsubsection{Organizational Culture Factors}

Proficiency Culture. This organizational culture expects that staff will be competent and have up-to-date knowledge about clients and clients' wellbeing is the priority to them (Glisson, 2007). Proficiency culture was not a significant predictor of overall models, but in the regression by domain it had a significant relationship with employment service provision. This was a similar result to a previous study. Olin et al. (2014) examined the relationship between organizational social contexts (organizational culture) of a program and the quality of the family support program. They found that programs with a highly proficient culture are more likely to provide a higher quality of practice.

The employment service domain includes job training and employment support. Normally, job training manuals exist to explain how to provide training service. However, service providers could provide additional training or job opportunities if they want. Moreover, in the researcher's experience, employment support such as job 
interview opportunities heavily depends on the service provider's attention. Therefore, proficiency organizational culture might help to enhance service providers' attention.

Rigid Culture. A rigid culture expects that staff provide limited input into management decisions and are controlled by agency rules and regulations (Glisson, 2007). This study found rigid culture was not a significant predictor of overall models, but it was negatively associated with assistance to access benefits and employment service referral. This is a similar finding as past studies. In the Olin et al. (2014) study, highly rigid cultures were more likely to be associated with poor performance. This result is quite predictable. Service providers who work under a rigid culture agency do not have enough flexibility to provide service referral because they should follow agency rules and procedures for service referrals.

\subsection{Contribution to the Theories/Models}

This study merged three theories: the gateway provider model (GPM; Stiffman, Pescosolido, \& Cabassa, 2004), the cultural competence model (CCM; CampinhaBacote, 2002; Giger \& Davidhizar, 2013; Jeffreys, 2010; NASW, 2001; Smith, 1998; Suarez-Balcazar \& Rodakowski, 2007; Suh, 2004), and the organization theory (organizational culture; Glisson et al., 2008b) to construct the conceptual framework as a theoretical tool to examine service providers' personal factors and organizational factors associated with refugee service providers' service provision and referral. The findings of this study provide support for all three of these theories.

This study specified the notion of the GPM as a major theoretical framework and CCM also support that service providers' personal factors are key factors in service 
provision. Stiffman, Pescosolido, and Cabassa (2004) mainly focused on the role of key person's capability, such as providers' amount of information, and their attitude and found individuals' abilities were important factors to providing mental health services to mentally ill adolescents. Findings from this research indicate that service providers' knowledge of refugee, familiarity with community resources, and cultural competence level as a personal capability were significant factors in predicting service provision and referral. Service providers who have a higher degree of knowledge of refugees, familiarity with community resources, and cultural competence behavior have a higher chance of providing and referring service to refugee clients. The mediating associations of the cultural competence also support the GPM and CCM stating that there is an interrelationship among multiple personal factors that influence service providers' service provision and referral.

The findings of this study are also consistent with organization theory. Furthermore, this study specified the organizational culture by investigating influence factors on service providers' decision behavior when they provide service to refugees. The results showed proficiency and rigid organizational culture were directly related to service providers' service provision and referral. Flexible organizational culture supports service providers' service provision, while bureaucratic organizational culture hampers service providers' service provision.

Conclusively, this merged theory based on the three theories/models (i.e., gateway provider model, cultural competence model, and organizational theory) makes this study unique. Using a merged theory/model, this study examined personal and organizational domains and identified how the multiple determinants were related to refugee service 
providers' service provision. Particularly, this study emphasized that using GPM showed the importance of personal capability in the process of providing service. In addition, this study highlighted service providers' cultural competence as a crucial personal capability and found significant interrelated relationships in predicting service providers' service provision. Lastly, service provision environmental determinants were also explained through adding organizational culture factor as organizational theory. This showed how service providers were influenced by organizational expectations and the way things were done when they provide service to refugees.

\subsection{Implications}

The primary purpose of this study was to identify the best predictors of service providers' personal factors and organizational culture factors in their service provision and referral to refugee clients. The findings of this study have implications for refugee service practice for providers and organizations. Furthermore, there are policy improvement implications to better support refugee service providers and organizations.

\subsubsection{Implication for Practice}

Actively Attend Cultural Competence Training: Individual Level. The findings, in this study, presented the critical role of service providers in facilitating service provision for refugees. Moreover, in this role, the results indicated service providers' cultural competence level was a key factor when they provide service to refugee clients. According to the study of Phillips (2004), refugees considered service

providers as instrumental to their resettlement adjustment. Therefore, if they did not have support related to cultural differences because of the service provider's lack of cultural 
competence, their adjustment process was negatively impacted. In addition, Keyser, Gamst, Meyers, Der-Karabetian, and Morrow (2014) mentioned culturally competent service providers who are knowledgeable and aware of the cultural characteristic of their clients may contribute to increased service provision. Therefore, refugee service providers should be aware of cultural issues and try to develop their cultural competence. It should enhance further intercultural work, such as advocacy. Furthermore, refugee service providers who are culturally competent can play a part in the generation of policy and participate in community efforts to increase refugee access to services. As such, to provide appropriate assistance to refugees so they successfully adjust, service providers should actively attend cultural competence training.

\section{Actively Support Cultural Competence Training: Organization Level. In}

Harrison and Turner's (2011) study, staff participants discussed the organizational constraints that impacted their ability to provide culturally competent service. Lack of organizational cultural competence can be a strong influence on service provision. Even if service providers wish to be more flexible in their service delivery, they may be constrained by an organization's policies and resources (Truong, Gibbs, Paradies, \& Priest, 2017). Hence, refugee service organizations should adopt more culturally competent practice in order to deliver appropriate service to refugees.

The findings clearly suggest that the cultural competence of the refugee service provider have an important role in providing services. Moreover, it might best be achieved through continuous professional education and training programs at an individual level. Therefore, at the organizational level, it is recommended that organizations offer routine cultural competence training and provide attendance 
incentives. In addition, organizations should try to hire bilingual and bicultural staff, such as former refugees, to reduce language barriers.

\subsubsection{Implication for Policy}

Cultural Competence Training Program for Service Providers. Service providers' level of cultural competence is important in order to provide service to refugees. However, there is no mandatory training requirement for refugee service providers. Licensed social workers, for example, undergo a certain amount of training to maintain their license, but there is no license for refugee service providers that includes a training requirement. So that refugee service providers understand refugees who have very unique backgrounds and they are able to provide appropriate service to them, it is recommended that there is a regulation for them to complete cultural competence training. Moreover, service providers who are considered gatekeepers are instrumental to resettlement adjustment for refugees. Therefore, if refugee clients are not offered appropriated support and culturally responsive service, their successful resettlement into a new place may take longer and may create difficulties. In order to work with this population, cultural competence is needed for refugee service providers. As this study has found, service providers who have a high level of cultural competence provided and referred more services to refugees. Due to this finding, it seems reasonable to suggest that a cultural competence training program would be necessary for refugee service providers to provide more service. There are several studies that show the benefits of a training program. A study by Uribe Guajardo et al. (2018) evaluated the effectiveness of mental health training for 86 service providers who provided service to Iraqi refugees in Australia. After three 7-hour class style interventions, the results (pre, post, and follow- 
up) demonstrated a significant impact on most measures such as knowledge of mental health problems and participant's confidence in helping an Iraqi refugee. Moreover, the Loya (2011) study found statistically significant results that white American practitioners who were more aware of racial awareness were less likely to be aware of racial privilege before receiving cultural diversity training.

Therefore, it is important and necessary to establish a cultural competence training program to support refugee service providers' service provision. Cultural competence training will provide a learning platform where service providers can access culturally appropriate resources or approaches to improve their understanding of refugees. Further, participants will learn the cultures of each refugee ethnic group and the history and politics of refugee homelands. It would likely enhance the ability of all refugee service providers to provide better services.

\section{Develop an Online Network System that Shares Information among}

Agencies. To provide effective service to refugee clients and to create long-term sustainability of refugee communities, it is helpful to develop an online system for each state, region, or area to share the same information. As this current study found, familiarity with community resources was a significant predictor of service provision to refugees. Service providers who are more familiar with community resources provided more service than those who did not. Therefore, if there are policies that enhance providers' connections with shared knowledge about refugee service resources or helpful information, this would likely affect service provision. Two benefits could be expected by having an online network system. 
First, using a shared online information system, service providers' workload should decrease, allowing them to provide more services to refugees. This is because they may save time in searching for information and the information should be more reliable. Moreover, using the shared information, service providers should be able to educate themselves about eligibility for certain services so they will know whether or not refugees are able to access them. Thus, knowledge of refugee resources can be enhanced and provider ability to provide accurate service to refugees will increase.

Secondly, the online system could create networking among community organizations. Refugees are frequently confused and overwhelmed by paperwork and a fragmented service system that shuffles them back and forth between agencies (Phillips, 2004). Lack of communication among agencies and no existing shared information could make this situation worse. During this process, a refugee might give up trying to obtain needed services and have difficulties adjusting to their new life as a result. Therefore, if an online network system helps integrate the steps and provide shared information, refugees could obtain services more easily. Moreover, an online network could be used as a referral pathway. Service providers could possibly identify needed services using the network system and refer refugees to other agencies where services are provided. Refugees might not need to go back and forth between agencies and may be less likely to give up trying to access needed services.

Therefore, the online network system among refugee service organizations working in a regional area needs to be developed to enable better information sharing, resulting in improved services for the refugees. 


\subsection{Strengths, Limitations and Recommendations for Future Research}

\subsubsection{Strengths of the Study}

A major strength of this study is primary data collection of refugee service providers. There are few research studies about refugee service providers, and most of them used a quantitative research method. In-depth and semi-structured interviews has provided insight into personal experiences and perceptions, but they could not give general information about the refugee service providers. This study utilized a quantitative research method to discover the more general issues regarding service providers who work with refugees. This unique data may help develop universal guidance toward specific and effective intervention strategies for refugee service providers as well as their organizations.

Another strength of this study include the examination of multiple (i.e., personal and organizational) determinants of refugee service providers. Using the merged theory, the study specified and extended how the multiple determinants were interactively associated with refugee service providers' service provision and referral. These associations were useful on multiple linear regression and PROCESS that allowed the examination of mediating effects. No other studies have examined the mediation effect of cultural competence in a study of refugee service providers.

\subsubsection{Limitation of the Study and Implication for Future Research}

There are several limitations in the present research that should be discussed. The foremost limitation is the sample size. This project was supposed to include a sample size of 122 for multiple regression analysis. However, an unexpected political situation happened. On January 27, 2017, the Trump administration's executive order on refugee 
travel threatened to halt service providers' efforts. This executive order suspended the entire refugee resettlement program for 120 days and barred entry to refugees from six countries — Syria, Iran, Sudan, Libya, Somalis, and Yemen—for 90 days. Moreover, the program will be capped at 50,000 refugees for fiscal year 2017, down from the 110,000 celling put in place under President Barack Obama (The New York Times, 2017). This is because the Trump administration and other US officials cited unfounded fears that terrorists may infiltrate the refugee resettlement program (International Rescue Committee [IRC], 2017). For those reasons, the number of refugees arriving in the US has declined. Most of the refugee agencies' programs are funded by the government, and this funding comes in a one-time, per-refugee amount, most of which gets used for direct expenses for newly arrived refugees during their first 90 days in the country. It includes covering rent costs for the family's apartment and a caseworker (The Washington Post, 2017). Trump's refugee ban means the agencies have to find nongovernmental funding to cover the budget to pay refugee service providers. Therefore, the government's decision not only affected the number of refugees allowed into the US, but also the number of providers (Newsweek, 2017, 5/25). Below are several news clips showing how the government decision affected service providers.

In Pennsylvania, which saw a 40 percent drop in refugee resettlement, several local agencies have noticed the decline in numbers. One agency, the Northern Area Multi Service Center, saw its revenues decline and laid off three workers. (Newsweek, 2017, 5/25) 
An evangelical relief and development agency that works with the United Nations' refugee program announced Wednesday that it will lay off 140 staff members and close five offices. (The Washington Post, 2017, 2/15)

InterFaith Works of Central New York said Trump's planned executive order will force the organization to shut down most of its Center for New Americans and lay off or cut back hours for 14 to 15 staffers.

(Syracuse.com, 2017, 1/26)

This research uses a sample size of 115 as the final number for multiple regression analysis. Small sample size may reduce the chance of detecting a true effect and also reduce statistically significant results. Thus, because of sample size, findings from this study may not provide as good of an explanation about refugee service providers and their service provision. Therefore, future studies will need to gather more data from a broader cross section of service providers of refugee communities.

Secondly, the understanding of service provision and referral from the research finding and the causal relationship among study variables was prevented due to the crosssectional design of the study. Moreover, the study utilizes a non-probability purposive sampling method, which limits the generalizability of the research findings. Even though this research collected data from across the States, the findings from a sample size of only 115 may not be generalized to all refugee service providers in the U.S. For instance, it is likely that service providers have varying service provisions or referrals for refugees. Thus, the sample represented only a small portion of the total numbers of refugee service 
providers in the U.S. Taken together, future studies will need to utilize a longitudinal design with a random sampling method, to examine causality between the predictors and service provision and referral. Use of a probability sampling will increase the generalizability of the study.

Thirdly, the study has the demographic homogeneity of the sample. Out of 115 total refugee service provider participants, there were only 32 other race/ethnicity (27.8\%), 21 males (18.3\%), and 18 service providers who were former refugees (15.7\%). According to census data, $67.3 \%$ of social workers are white (Datauas, 2015), and the average percent of female social service professionals is $86 \%$ (The Boston Blode, 2017, 3/7). Therefore, the ratio of white and non-white and male and female from this study sample looks similar to the national average. There was no information on the national average percent of former refugee status, but it may be assumed not many. Although homogeneity of race/ethnicity, sex, and former refugee status was average in this study, these small subgroups hinder the representativeness of the sample and limit the ability to detect potentially significant findings. For example, this study may have had different service provision among minority groups, such as minority service providers are more likely than their counterparts to provide more effective service to refugee clients. However, study findings might not detect those differences because the minority subgroups are drastically underrepresented in refugee service providers. Therefore, future research should set up the study design carefully to consider balancing the sample characteristics. This would help to provide a better understanding and diverse view of refugee service providers' service provision. 
Fourthly, the study utilized non-standardized measurements for knowledge of refugee and familiarity with community resources. There were no existing yardsticks to measure refugee service providers' knowledge of refugees and familiarity with community resources. Therefore, the researcher created it based on several resources. These measurements did not include enough data that could explain refugee service provisions. The measurement of knowledge of refugees was focused on general overview of refugees rather than specific details such as refugees' health related questions (substance abuse, mental disorder, and addiction). Therefore, specialized knowledge could not be obtained through the measurement. Moreover, for familiarity with community resource measurement, it included initial resettlement-related community resources, such as basic benefits (Medicaid, school enrollment, and TANF program), clinics (mental health and church), and specialists (social worker and counselor). Those resources seemed common and easily accessible; however, service providers working at places like rural areas, where there is a lack of resources, might not be able to do this. The score of refugee service providers in the rural areas would be lower than that of their urban counterparts. However, it does not mean that rural area service providers are not familiar with community resources. They just may not have any resources around them. Both the measurements could not include these exceptional situations. Therefore, future studies should continue to expand on the knowledge of refugees, including specialized knowledge of refugee enabling predicting service provision and referral better. Moreover, familiarity with community resources should be measured based on where the service provider is providing service, to arrive at earn accurate results in future studies. 
Fifthly, this study may have recall bias when participants answered survey questions. Especially for dependent variable, service provision and referral, the participants were asked 'How many times you have been provided/referred the following services in the past 12 months?' and the possible selection options were 0 to 6 , each indicating certain number of services provided and referred. However, the service provider may not remember how many services they had provided for the past year due to their heavy work load. This is because, they may provide several services which could be hard to count. Therefore, future research should set up the survey questionnaire carefully to consider the most measurable. This would help to detect exactly what is supposed to be measured and finally it would help obtain exact findings of refugee service providers' service provision.

Lastly, this study may not explain enough about organizational factors. In this study, organizational culture factors were considered one of the important predictors to explain refugee service provision and referral. However, this study was more focused on individual service providers' personal factors, thus organizational culture factors did not explain enough. Although, service providers' personal factors were important for predicting service provision, organizational climate could affect service providers' service provision. For example, depending on an organization's capability, refugee service providers could have a support system that overcomes the difficulties they often encounter. Thus, they may provide greater service to refugee clients. Therefore, future studies should continue to expand on the influence of organizational factors on the relationship between refugee service providers and their service provision. To determine those influences, future studies should collect data by type of organization, such as 
religious, government, health, or others, and then compare their differences in amount of service provision to refugees by type of organization factor. The important organizational factors would be found to explain service provision to refugees. Findings will give to refugee service providers and organizational administrators realistic recommendations about proper organization environment to provide services to refugees.

Regardless of any limitations, this study can be an initial attempt to understand predictors of refugee service provisions and can be an important step toward the development and implementation of programs and policies on behalf of this growing population.

Additional recommendations for future research need to be discussed. The first is for future research on refugees' need for services and their receipt of service. This study investigated which factors were important when refugee service providers provided service to refugees. Service providers' personal factors, such as knowledge of refugee, familiarity with community resources, and cultural competence, and organizational culture factors, such as proficiency and rigidity culture, were found to be significant factors. Moreover, refugee service providers mentioned that case management, transportation, and cultural orientation were the most frequently provided services to refugees. For referral, health insurance support, health screening support, and medical appointment support were the most frequently referred to refugees. However, we are not sure these services are what refugees expect or need. Moreover, this study only measured service quantity and not quality. Service quality could be measured by service recipients such as refugees. Therefore, the next research step would be to investigate the refugee perspective to fill the gap. More specifically, it is necessary to be aware of what services 
refugees need. The opinion of needs may differ between refugees and service providers. After that, service quality needs to be measured. This study has shown the refugee service providers' perspective, and future research could be conducted to ascertain the perspective of refugees, which would be helpful to understand refugee issues as a whole.

The second recommendation for future study is focusing on refugee service providers' cultural competence. One of the major findings of this study is the role of cultural competence. As presented on mediation results, cultural competence, especially cultural competence behavior, was a significant key element that could account for service providers' service provision. Service provider's work experience did not have any relationship with service provision and referral. However, put cultural competence behavior between work experience and service provision as a mediator and the association appears. However, more detailed and direct information to support the importance of cultural competence may be helpful to create cultural competence training programs. For future study, therefore, I suggest experimental research using pre-test and post-test self-reported forms. With this experiment, we can better understand the role of cultural competence and develop appropriate cultural competence programs for refugee service providers. As refugee service providers grow in their level of cultural competence, refugees stand to gain more culturally appropriate services.

Lastly, a recommendation for future study is to focus on the barriers that refugee service providers face when they provide service to refugees. This study focused on the predictors of characteristics of service providers. It is important to know what personal predictors could affect greater service provision, but to understand service providers' challenges in refugee service provision is also important. Therefore, I suggest conducting 
a study of refugee service providers' challenges to obtain better information on refugee service provision in the field.

\subsection{Conclusion}

Refugees who are forced to move encounter many challenges in adjusting and adapting to their new life, sometimes with very little or too much information. In their life, service providers are often their first point of contact for assistance, providing some immediate services and help navigating the US system. Therefore, this research focused on exploring important determinants of refugee service providers when they provide or refer service to refugee clients. The study also examined the cultural competence mediation effect on the association between familiarity with community resources and work experience and service provision and referral.

The research was not only guided by theory but also offers evidence to add to current theory in service providers' service provision. The findings from this study lend strong support for the gateway provider model for assessing the importance of service providers' personal factors. Moreover, this research included a sample of refugee service providers and explored important predictors of service provision. The unique findings surpass much of the prior research on refugees and refugee service providers. Therefore, this research contributes to an understanding of refugee service providers and their service provision as well as provides important information for refugee service providers, refugee service organizations, and policy makers to help guide practice and training program development.

In conclusion, the present study may provide the foundation for future research 
and expand the information on refugee service providers, which is understudied. Finally, refugee service providers, community leaders, public officials, and policy makers should work closely for the refugee population to develop the best strategies for enhancing refugees' quality of life as they transition to a new living environment. 


\section{REFERENCES}

Allison, P. D. (2002). Missing data. Thousand Oaks, CA: Sage Publications.

Aarons, G. A., \& Sawitzky, A. C. (2006). Organizational climate partially mediates the effect of culture on work attitudes and staff turnover in mental health services. Administration and Policy in Mental Health and Mental Health Services Research, 33(3), 289-301.

Aarons, G. A., Gllisson, D., Green, P. D., Hoagwood, K., Kelleher, K. J., Landsverk, J. A., \& The Research Network on Youth Mental Health. (2012). The organizational social context of mental health services and clinician attitudes toward evidencebased practice: A United States national study. Implementation Science, 7, 56-71.

American Immigration Council. (2015). An overview of U.S. refugee law and policy.

Retrieved from

http://www.immigrationpolicy.org/sites/default/files/docs/an overview of u.s. re fugee law and policy.pdf

Andersen, R. N. (1995). Revisiting the behavioral model and access to medical care: Does it matter? Journal of Health and Social Behavior, 36 (March), 1-10.

Aragaw, A., Yigzaw, T., Tetemke, D., \& G/Amlak, W. (2015). Cultural competence among maternal healthcare providers in Bahir Dar city administration, Northwest Ethiopia: Cross sectional study. BMC Pregnancy and Childbirth, 15, 227-236. http://doi.org/10.1186/s12884-015-0643-8

Araya, M., Chotai, J., Komproe, I. H., \& de Jong, J. T. (2007). Effect of trauma on quality of life as mediated by mental distress and moderated by coping and social 
support among postconflict displaced Ethiopians. Quality of Life Research, 16, 915-927.

Bailey, T. J. (n.d). To the next step...moving from Baron and Kenny to bootstrapping in mediation analysis. Retrieved from https://tomjamesbailey.wordpress.com/2014/01/03/to-the-next-step-movingbeyond-baron-and-kenny-in-mediation-analysis/

Baker, J. (2013). Just kids? Peer racism in a predominantly white city. Refugee: Canada's Journal on Refugee, 29, 75-85.

Balaghi, D. A., Westdal, J. N., \& Rispoli, K. (2017). Social context during resettlement and its influence on Arab refugee youth's well-being. School Psychology Review, 46(4), 145-159.

Bandura, A. (1986). Social foundations of thought and action: A social cognitive theory. Englewood Cliffs, NJ: Prentice-Hall.

Baron, R. M., \& Kenny, D. A. (1986). The moderator-mediator variable distinction in social psychological research: Conceptual, strategic and statistical considerations. Journal of Personality and Social Psychology, 51(6), 1173-82.

Bayram, N., Thorburn, D., Demirhan, H., \& Bilgel, N. (2007). Quality of life among Turkish immigrants in Sweden. Quality of Life Research, 16(8), 1319-1333.

Beach, M. C., Price, E. G., Gary, T. L., Robinson, K. A., Gozu, A., Palacio, A. ... Cooper, L. A. (2005). Cultural competence: A systematic review of health care provider educational interventions. Medical Care, 43(4), 356-373.

doi:10.1097/01.mlr.0000156861.58905.96 
Beiser, M., \& Hou, F. (2001). Language acquisition, unemployment and depressive disorder among Southeast Asian refugees: A 10-year study. Social Science and Medicine, 53, 1321-1334.

Beiser, M. (2005). The health of immigrants and refugees in Canada. Canadian Journal of Public Health, 96(2), S30-S44.

Berns-McGown, R. (2013). “I am Canadian”: Challenging stereotypes about young Somali Canadian. IRPP Study, 38, 1-30.

Betancourt, J. R., Green, A. R., Carrillo, J. E., \& Park, E. R. (2005). Cultural competence and health care disparities: Key perspectives and trends. Health Affairs, 24(2), 499-505. doi:10.1377/hlthaff.24.2.499

Betancourt, J. R., \& Green, A. R. (2010). Commentary: Linking cultural competence training to improved health outcomes: Perspectives from the field. Academic Medicine, 85(4), 583-585.

Birman, D., Trickett, E. J., \& Vinokurov, A. (2002). Acculturation and adaptation of Soviet Jewish refugee adolescents: Predictors of adjustment across life domains. American Journal of Community Psychology, 30, 585-607.

Black, R. (2001). Environmental refugees: Myth or reality? In New Issues in Refugee Research, Working Paper (34), United Nations High Commissioner for Refugees (UNHCR), Geneva.

Brown, A., \& Scribner, T. (2014). Unfulfilled promises, future possibilities: The refugee resettlement system in the United States. Journal of Migration and Human Security, 2(2), 101-120. 
Brunette, M., Asher, D., Whitley, R., Lutz, W., Wieder, B., Jones, A., et al. (2008). Implementation of integrated dual disorders treatment: A qualitative analysis of facilitators and barriers. Psychiatric Services, 59(9), 989-995.

Bunger, A. C., Stiffman, A. R., Foster, K. A., \& Shi, P. (2009). Child welfare workers' connectivity to resources and youth's receipt of services. Advances in Social Work, 10(1), 19-38.

Bunger, A. C. (2010). Defining service coordination: A social work perspective. Journal of Social Service Research, 36(5), 385-401.

Bureau of Population, Refugees, and Migration. (2017). Proposed Refugee Admission FY 2018 Report. Retrieved from https://www.state.gov/documents/organization/274857.pdf

Burke, M. I., Landis, R. S., \& Burke, M. J. (2017). Estimating group-level relationships: General recommendations and considerations for the use of intraclass correlation coefficients. Journal of Business and Psychology, 32, 611-626.

Calsyn, R. J., \& Roades, L. A. (1993). Prediction perceived service needs, service awareness, and service utilization. Journal of Gerontological Social Work, 21(1/2), 59-76.

Campinha-Bacote, J. (2002). The process of cultural competence in the delivery of healthcare services: A model of care. Journal of Transcultural Nursing, 13(3), 181-184. doi:10.1177/10459602013003003

Carlsson, J. M., Mortensen, E. L., \& Kastrup, M. (2006). Predictors of mental health and quality of life in male tortured refugees. Nordic Journal of Psychiatric, 60, 51-57. 
Carson, C. M. (2005). A history view of Douglas McGregor's Theory Y. Management Decision, 43(3), 450-460.

Cooper, L. A., \& Roter, D. L. (2002). Patient-provider communication: The effect of race and ethnicity on process and outcomes of healthcare. In B. D. Smedley, A. Y. Stith, \& A. R. Nelson (Eds.), Unequal treatment: Confronting racial and ethnic disparities in healthcare (pp. 552-593). Washington, DC: The National Academies Press.

Copeland, V. (2006). Disparities in mental health service utilization among low-income African American adolescents: Closing the gap by enhancing practitioner's competence. Child \& Adolescent Social Work Journal, 23(4), 407-431.

Clegg, S., Kornberger, M., \& Pitsis, T. (2011). Managing \& organizations: An introduction to theory \& practice. Thousand Oaks, CA: SAGE.

Cross, T. L., Bazron, B. J., Dennis, K. W., \& Issacs, M. R. (1989). Towards a culturally competent system of care: A monograph on effective service for minority children who are severely emotionally disturbed. Washington, DC: Georgetown University Child Development Center.

Cultural Orientation Resource Center. (n.d.). About cultural \& community orientation. Retrieved from http://www.culturalorientation.net/providing-orientation/about Datausa. (2015). Datausa: Social workers. Retrieved from https://datausa.io/profile/soc/211020/\#demographics de Figueiredo, S., Yetwin, A., Sherer, S., Radzik, M., \& Iverson, E. (2014). A crossdisciplinary comparison of perceptions of compassion fatigue and satisfaction 
among service providers of highly traumatized children and adolescents.

Traumatology: An International Journal, 20(4), 286-295. doi:10.1037/h0099833

Delobbe, N., Haccoun, R. R., \& Vandenberghe, C. (2002). Measuring core dimensions of organizational culture: A review of research and development of a new instrument. Unpublished manuscript, Universite catholique de Louvain, Belgium.

Dempster, A. P., Laird, N. N., \& Rubin, D. B. (1977). Maximum likelihood from incomplete data via the EM algorithm. Journal of the Royal Statistical Society. Series B (Methodological), 39, 1-38.

Denison, D. R. (1996). What is the difference between organizational culture and organizational climate? A native's point of view on a decade of paradigm wars. The Academy of Management Review, 21(3), 619-654.

doi:10.5465/AMR.1996.9702100310

Department of State (2016). Refugee arrival by state. Retrieved from http://www.wrapsnet.org/Portals/1/arrivals/Arrivals\%20by\%20State\%20-020Ma p(9.16.16).pdf?ver=2016-09-16-101232-627

Ding, W., \& Song, P. X.-K. (2016). EM algorithm in Gaussian copula with missing data. Computational Statistics and Data Analysis, 101, 1-11.

Do, C. B., \& Batzoglou, S. (2008). What is the expectation maximization algorithm? Nature Biotechnology, 26(8), 897-899.

Doorenbos, A. Z., Schim, S. M., Benkert, R., \& Borse, N. N. (2005). Psychometric evaluation of the cultural competence assessment instrument among healthcare providers. Nursing Research, 54(5), 324-331. 
Drachman, D. (1995). Immigration statuses and their influence on service provision, access, and use. Social Work, 40(2), 188-197. doi:10.1093/sw/40.2.188

Drachman, D., \& Ryan, A. S. (2001). Immigrants and refugees. In A. Gitterman (Ed). Hanbook of social work practice with vulnerable and resilient populations $\left(^{\text {nd }}\right.$ ed.). New York: Columbia.

Edmonds, J. K., Hruschka, D., Bernard, H. R., \& Sibley, L. (2012). Women's social networks and birth attendant decisions: Application of the Network-Episode Model. Social Science \& Medicine, 74(3), 452-459.

Ehrhart, M. G., Schneider, B., \& Macey, W. H. (2014). Organizational climate and culture: An introduction to theory, research, and practice. New York, NY: Routledge.

Elliott, S., \& Yusuf, I. (2014). 'Yes, we can: but together': Social capital and refugee resettlement. Kotuitui, 9, 101-110.

Frey, W. H. (2015). Diversity explosion: How new racial demographics are remaking America. Washington, D.C: Brookings Institution Press.

Forbes, G. M. (2012, July 5). Classical organizational theory, behavioral school and contemporary theories of management. Retrieved from http://schoolprincipal.blogspot.com/2012/07/classical-organizational-theory.html

Fox, S., \& Tang, S. (2000). The Sierra Leonean refugee experience: Traumatic events and psychiatric sequelae. Journal of Nervous and Mental Disease, 188, 490-495.

Giger, J. N., \& Davidhizar, R. E. (2013). Transcultural nursing: Assessment \& intervention ( $6^{\text {th }}$ ed.). St. Louis, MO: Mosby. 
Glisson, C. (2002). The organizational context of children's mental health services. Clinical Child and Family Psychology Review, 5(4), 233-253.

Glisson, C. (2007). Assessing and changing organizational culture and climate for effective services. Research on Social Work Practice, 17(6), 736-747.

Glisson, C. (2010). Organizational climate and service outcomes in child welfare settings. In M. B. Webb, K. L. Dowd, B. J. Harden, J. Landsverk, \& M. Testa (Eds.), Child welfare and child well-being: New perspectives from the National Survey of Child and Adolescent Well-being. New York: Oxford University Press.

Glisson, C., \& Green, P. (2006). The effects of organizational culture and climate on the access to mental health care in child welfare and juvenile justice systems. Administration and Policy in Mental Health and Mental Health Services Research, 33(4), 433-448.

Glisson, C., Green, P., \& Williams, N. J. (2012). Assessing the organizational social context (OSC) of child welfare systems: Implications for research and practice. Child Abuse and Neglect, 36(9), 621-632. doi:10.1016/j.chiabu.2012.06.002

Glisson, C., \& James, L. R. (2002). The cross-level effects of culture and climate in human service teams. Journal of Organizational Behavior, 23, 767-794.

Glisson C., Landsverk J., Schoenwald, S. K., Kelleher, K., Hoagwood, K. E., Mayberg, S., \& Green, P. (2008a). Assessing the organizational social context (OSC) of mental health services: Implications for research and practice. Administration and Policy in Mental Health and Mental Health Services Research, 35(1-2), 98-113.

Glisson, C., Green, P., Kelleher, K., Landsverk, J., Hoagwood, K. E., \& ... Schoenwald, S. K. (2008b). Therapist turnover and new program sustainability in mental health 
clinics as a function of organizational culture, climate, and service structure. Administration and Policy in Mental Health and Mental Health Services Research, 35(1), 124-133. doi:10.1007/s10488-007-0152-9

Glisson, C., Williams, N. J., Green, P., Hemmelgarn, A., \& Hoagwood, K. (2014). The organizational social context of mental health Medicaid waiver programs with family support services: Implications for research and practice. Administration and Policy in Mental Health and Mental Health Services Research, 41(1), 32-42. doi:10.1007/s10488-013-0517-1

Griswold, K., Zayas, L. E., \& Kernan, J. B. (2007). Cultural awareness through medical student and refugee patient encounters. Journal of Immigrant and Minority Health, 9, 55-60, doi: 10.1007/s10903-006-9016-8

Guzzo, R. A., Jette, R. D., \& Katzell, R. A. (1985). The effects of psychologically based intervention programs on worker productivity: A meta-analysis. Personnel Psychology, 38(2), 275-291.

Habeck, C. G., \& Brickman, A. M. (2014). A common statistical misunderstanding in Psychology: Do we need normally distributed independent or dependent variables for linear regression to work? Retrieved from http://www.columbia.edu/cu/bnet/StatisticalFallacy July2014.pdf

Hadfield, K., Ostrowski, A., \& Ungar, M. (2017). What can we expect of the mental health and well-being of Syrian refugee children and adolescents in Canada? Canadian Psychology/Psychologie Canadienne, 58(2), 194-201.

Håkonsen, H., Lees, K., \& Toverud, E. (2014). Cultural barriers encountered by Norwegian community pharmacists in providing service to non-western 
immigrant patients. International Journal of Clinical Pharmacy, 36(6), 1144-

1151. doi:10.1007/s11096-014-0005-z

Hanniman, W. (2008). Canadian Muslim, Islamophobia and national security. International Journal of Law, Crime and Justice, 36, 271-285.

Harrison, G., \& Turner, R. (2011). Being a 'culturally competent' social work: Making sense of a murky concept in practice. British Journal of Social Work, 41, 333-350.

Hart, P. L., \& Mareno, N. (2016). Nurses' perception of their cultural competence in caring for diverse patient populations. Online Journal of Cultural Competence in Nursing and Healthcare, 6(1), 121-137.

Hayes, A. F. (2009). Beyond baron and Kenny: Statistical mediation analysis in the new millennium. Communication Monographs, 76(4), 408-420.

Hayes, A. F. (2012). PROCESS: A versatile computational tool for observed variable mediation, moderation, and conditional process modeling [White paper]. Retrieved from http://www.afhayes.com/public/process2012.pdh.

Hayes, A. F. (2013). Introduction to mediation, moderation, and conditional process analysis: A regression-based approach. New York, NY: Guilford Press.

Health Resources and Services Adminiatration. (2011). 2011 report to congress: National strategy for quality improvement in health care.

Henry, H. M. (2012). African refugees in Egypt: Trauma, loss, and cultural adjustment. Death Studies, 36(7), 583-604.

Hodge, B. J., \& Anthony, W. P. (1988). Organization theory (3rd ed.). Boston, MA: Allyn and Bacon. 
Hughes, J., Barker, D., Kemenoff, S., \& Hart, M. (1993). Problem ownership, causal attributions, and self-efficacy as predictors of teachers' referral decisions. Journal of Educational and Psychological Consultation, 4, 369-384.

International Rescue Committee. (2017, January 17). What is the travel ban? What does it mean for refugees? Retrieved from https://www.rescue.org/article/what-travelban-what-does-it-mean-refugees

James, L. A., \& James, L. R. (1989). Integrating work environment perceptions: Explorations into the measurement of meaning. Journal of Applied Psychology, 74(5), 739-751.

Jewson, A., Lamaro, G., Crisp, B., Hanna, L., \& Taket, A. (2015). Service providers' experiences and needs in working with refugees in the Geelong region: A qualitative study. Australian Journal of Primary Health, 21(2), 233-238. doi:10.1071/PY12132

Jeffreys, M. R. (2010). A model to guide cultural competence education. In M. R. Jeffreys (Ed.), Teaching cultural competence in nursing and health care: Inquiry, action, and innovation ( $2^{\text {nd }}$ ed., pp. 45-59). New York, NY: Springer.

Johnson, D. R., Ziersch, A. M., \& Burgess, T. (2008). I don't think general practice should be the front line: Experiences of general practitioners working with refugees in South Australia. Australia and New Zealand Health Policy, 5, 20. doi:10.1186/1743-8462-5-20.

Kang, S. M. (2006). Measurement of acculturation, scale formats, and language competence: Their implications for adjustment. Journal of Cross-Cultural Psychology, 37(6), 669-693. 
Kast, F. E., \& Rosenzweig, J. E. (1996). General system theory: Applications for organization and management. In J. M. Shafritz \& J. S. Ott (Eds.), Classics of organization theory ( $4^{\text {th }}$ ed., pp. 302-315). Belmont: Wadsworth Pub. Co.

Kahn, A. J. (1979). Social policy and social services $\left(2^{\text {nd }}\right.$ ed.). New York: Random House.

Kavanagh, K., Absalom, K., Beil, W., \& Schliessmann, L. (1999). Connecting and becoming culturally competent: A Lakota example. Advanced in Nursing Science, 21, 9-31.

Kenny, P., \& Lockwood-Kenny, K. (2011). A mixed blessing: Karen resettlement to the United States. Journal of Refugee Studies, 24(2), 217-238. doi:10.1093/jrs/fer009

Keyes, E. F., \& Kane, C. F. (2004). Belonging and adapting: Mental health of Bosnian refugees living in the United States. Issues in Mental Health Nursing, 25(8), 809831.

Keyser, V., Gamst, G., Meyers, L. S., Der-Karabetian, A., \& Morrow, G. (2014). Predictors of self-perceived cultural competence among children's mental health providers. Cultural Diversity and Ethnic Minority Psychology, 20(3), 324-335.

Kincaid, D. L. (2004). From innovation to social norm: Bounded normative influence. Journal of Health Communication, 9(1), 37-57. doi:10.1080/10810730490271511

Kirmayer, L. J., Narasiah, L., Munoz, M., Rashid. M., Ryder, A. G., Guzder, J., et al. (2011). Common mental health problems in immigrants and refugees: General approach in primary care. Canadian Medical Association Journal, 183, E959-67. 
Kisa, S. (2015). The validity and reliability of the awareness scale regarding the Turkish adaptation for the cervical cancer and HPV infection among the women in fertility age [Abstract]. Journal of Nursing and Care, 4(4).

Kiteki, B. N. (2011). Acculturation and psychosocial adjustment of African adolescent refugees in the United States: The role of social support (Doctoral dissertation). The University of North Carolina at Greensboro.

Kline, R. B. (2015). The mediation myth. Basic and Applied Social Psychology, 37(4), 202-213.

Koberg, C. S., \& Chusmir, L. H. (1987). Organizational culture relationships with creativity and other job-related variables. Journal of Business Research, 15, 397409.

Kopelman, R. E., Prottas, D. J., \& Davis, A. L. (2008). Douglas McGregor's Theory X and Y: Toward a construct-valid measure. Journal of Managerial Issues, 20(2), 255-271.

Kovacev, L., \& Shute, R. (2004). Acculturation and social support in relation to psychosocial adjustment of adolescent refugees resettled in Australia. International Journal of Behavioral Development, 28(3), 259-267.

Kunkle, F. (2015, June 25). Census update shows more diverse society as baby-boom generation ages. The Washington Post. Retrieved from https://www.washingtonpost.com/local/social-issues/census-update-shows-morediverse-society-as-baby-boom-generation-ages/2015/06/24/03de1e24-1ab2-11e593b7-5eddc056ad8a story.html 
Larsen, R., \& Reif, L. (2011). Effectiveness of cultural immersion and culture classes for enhancing nursing students' transcultural self-efficacy. Journal of Nursing Education, 50(6), 350-354.

Lavik, N. J., Hauff, E., Skrondal, A., \& Solberg, Ø. (1996). Mental disorder among refugees and the impact of persecution and exile: Some findings from and outpatients population. British Journal of Psychiatry, 169, 726-732.

Leigh, J. W. (1998). Communicating for cultural competence. Boston: Allyn \& Bacon.

Little, R. A., \& Rubin, D. B. (1987). Statistical analysis with missing data. New York: Wiley.

Little, T. D. (2013). Longitudinal structural equation modeling. New York, NY: Guilford Press.

Loya, M. (2011). Color-blind racial attitudes in white social worker: A cross-sectional study. Smith College Studies in Social Work, 81, 201-217.

Mann, C. M., \& Fazil, Q. (2006). Mental illness in asylum seekers and refugees. Primary Care Mental Health, 4, 57-66.

Marotta, S. A. (2003). Unflinching empathy: Counselors and tortured refugees. Journal of Counseling and Development, 81, 111-114.

Martin, D. C., \& Yankay, J. E. (2013). Refugees and Asylees: 2012. Annual Flow Report of Department of Homeland Security.

McGregor, D. (1960). The human side of enterprise. McGraw Hill: London.

Migration Policy Institute. (2015). Refugees and asylees in the United States. Retrieved from http://www.migrationpolicy.org/article/refugees-and-asylees-united-states 
Miller, K. E., Weine, S. M., Ramic, A., Brkic, N., Bjedic, Z. D., Smajkic, A., Boskailo, E., \& Worthington, G. (2002). The relative contribution of war experiences and exile-related stressors to levels of psychological distress among Bosnian refugees. Journal of Trauma Stress, 15, 377-378.

Moe, J. F., \& Sparkman, N. M. (2015). Assessing service providers at LGBTQ- affirming community agencies on their perceptions of training needs and barriers service. Journal of Gay \& Lesbian Social Services, 27(3), 350-370.

Mollica, R. F., Sarajlic, N., Chernoff, M., \& Lavelle, J. (2001). Longitudinal study of psychiatric symptoms, disability, mortality and emigration among Bosnian refugee. Journal of the American Medical Association, 286, 546-554.

Morgeson, F. P., Dierdorff, E. C., \& Hmurovic, J. L. (2010). Work design in situ: Understanding the role of occupational and organizational context. Journal of Organizational Behavior, 31, 351-360.

Mukhtar, M. (2013). Settlement service providers in peel region, Ontario: Challenges, barriers and opportunities in the shadow state. (AAT 1571020)

Murray, E., \& Holmes, A. (2012). Addressing healthcare-associated infections and antimicrobial resistance from an organizational perspective: Progress and challenges. Journal of Antimicrobial Chemotherapy, 67(1), i29-i36. doi:10.1093/jac/dks200

Nalwadda, G., Mirembe, F., Tumwesigye, N. M., Byamugisha, J., \& Faxelid, E. (2011). Constraints and prospects for contraceptive service provision to young people in uganda: Providers' perspectives. BMC Health Services Research, 11(1), 220-220. doi:10.1186/1472-6963-11-220 
National Association of Social Workers (NASW). (2015). NASW standards for cultural competence in social work practice. Retrieved from https://www.socialworkers.org/practice/standards/naswculturalstandards.pdf

Newsweek. (2017, May 25). Drops significantly since Trump presidency. Retrieved from http://www.newsweek.com/refugee-resettlement-us-drops-trump-travel-ban$\underline{615569}$

O’Dwyer, L. M., \& Parker, C. E. (2014). A primer for analyzing nested data: Multilevel modeling in SPSS using an example from a REL study (REL 2015-046).

Washington, DC: U.S. Department of Education, Institute of Education Science, National Center for Education Evaluation and Regional Assistance, Regional Educational Laboratory Northeast \& Island. Retrieved from http://ies.ed.gov/ncee/edlabs.

Ogbu, J. U., \& Simons, H. D. (1998). Voluntary and involuntary minorities: A culturalecological theory of school performance with some implications for education. Anthropology \& Education Quarterly, 29(2), 155-188.

Olin, S. S., Williams, N., Pollock, M., Armusewicz, K., Kutash, K., Glisson, C., \& Hoagwood, K. E. (2013). Quality indicators for family support services and their relationship to organizational social context. Administration and Policy in Mental Health and Mental Health Services Research, 1-12. doi: 10.1007/s10488-0130499-Z

Oliver, B. D. (2014). Bhutanese refugees' health behaviors and perceptions: A qualitative approach to understanding Bhutanese refugees' post-resettlement experiences with health care services in middle Tennessee. (AAT 3636391) 
Olivier, C., \& Dykeman, M. (2003). Challenges to HIV service provision: the commonalities for nurses and social workers. Aids Care, 15(5), 649-663.

Onoka, C. A., Onwujekwe, O. E., Hanson, K., \& Uzochukwu, B. S. (2012). Sub-optimal delivery of intermittent preventive treatment for malaria in pregnancy in Nigeria: Influence of provider factors. Malaria Journal, 11(1), 317-317. doi:10.1186/1475-2875-11-317

Parker, C. P., Baltes, B. B., Young, S. A., Huff, J. W., Altmann, R. A., Lacost, H. A., et al. (2003). Relationships between psychological climate perceptions and work outcomes: A meta-analytic review. Journal of Organizational Behavior, 24(4), $389-416$.

Pescosolido, B. A. (1992). Beyond rational choice: The social dynamics of how people seek help. American Journal of Sociology. 97, 1096-1138.

Peterson, M. (2009). An introduction to decision theory [electronic resource] (1st ed.). Cambridge, UK: Cambridge University Press.

Pettigrew, A. M. (1979). On studying organizational cultures. Administrative Science Quarterly, 24(4), 570-581.

Pew Research Center. (2018). Key facts about refugees to the U.S. Retrieved from http://www.pewresearch.org/fact-tank/2017/01/30/key-facts-about-refugees-tothe-u-s/

Phillips, K. A. (2004). Intercultural knowledge and skills in social service work with refugee: Perspectives from providers and recipients of service (Doctoral dissertation). Retrieved from ProQuest Dissertations and Theses database. (UMI No. 3154788) 
Polit, D. F. (1996). Data analysis \& statistics for nursing research. Appleton \& Lange.

Preacher, K. J., \& Hayes, A. F. (2008). Asymptotic and resampling strategies for assessing and comparing indirect effects in multiple mediator models. Behavior Research Methods, 40(3), 879-891.

Procter, N. G. (2005). They first killed his heart (then) he took his own life. Part 1: A review of the context and literature on mental health issues for refugees and asylum seekers. International Journal of Nursing Practice, 11, 286-291.

Provan, K, G. (1984). Technology and interorganizational activity as predictors of client referrals. Academy of Management Journal, 27(4), 811-829.

Pumariega, A. J., Rothe, E., \& Pumariega, J. B. (2005). Mental health of immigrants and refugees. Community Mental Health Journal, 41(5), 581-597.

Raudenbush, S. W., \& Bryk, A. S. (2001). Hierarchical linear models: Applications and data analysis methods ( $2^{\text {nd }}$ ed.). Thousand Oaks, CA: Sage Publications.

Resnik, M. D. (1987). Choices: An introduction to decision theory. Minneapolis: University of Minnesota Press.

Rizzo, V. M. (2002). Social work support services for stroke patients: Interventions and outcomes (Doctoral dissertation). Retrieved from ProQuest Digital dissertations.

Refugee Council USA. (n.d.). History of the U.S. refugee resettlement program. Retrieved from http://www.rcusa.org/history.

Refugee Processing Center. (2018). Refugee Admissions Report. Retrieved from http://www.wrapsnet.org/admissions-and-arrivals/ 
Rooda, L. A. (1992). Attitudes of nurses toward culturally diverse patients: An examination of the social contact theory. Journal of National Black Nurses' Association, 6(1), 48-56.

Rosenheck, R., \& Lam, J. A. (1997). Homeless mentally ill clients' and providers' perceptions of service needs and clients' use of services. Psychiatric Services, $48(3), 381-386$.

Rucker, D. D., Preacher, K. J., Tormala, Z. L., \& Petty, R. (2011). Mediation analysis in social psychology: Current practices and new recommendations. Social and Personality Psychology Compass, 5, 359-371.

Sabbah, C. (2007). Social adjustment and idealized identity of refugee adolescents. International Journal of Humanities, 4, 117-125.

Sackmann, S. (1991). Cultural knowledge in organizations: Exploring the collective mind. Newbury Park, CA: Sage Club.

Salimbene, S. (1999). Cultural competence: A priority for performance improvement action. Journal of Nursing Care Quality, 13(3), 23-35.

Sam, D. L., \& Berry, J. W. (1995). Acculturative stress among young immigrants in Norway. Scandinavian Journal of Psychology, 36, 10-21.

Shen, Z. (2015). Cultural competence models and cultural competence assessment instruments in nursing: A literature review. Journal of Transcultural Nursing, $26(3), 308-321$.

Schim, S. A., Doorenbos, A. Z., Miller, J., \& Benkert, R. (2003). Development of a cultural competence assessment instrument. Journal of Nursing Measurement, 11(1), 29-40. 
Schim, S. M., Doorenbos, A. Z., \& Borse, N. N. (2005). Cultural competence among Ontario and Michigan healthcare providers. Journal of Nursing Scholarship, 37(4), 354-360.

Schneider, B., Ehrhart, M. G., \& Macey, W. H. (2013). Organizational climate and culture. Annual Review of Psychology, 64, 361-388.

Schwartz, S. J., Unger, J. B., Zamboanga, B. L., \& Szapocznik, J. (2010). Rethinking the concept of acculturation: Implications for theory and research. American Psychologist, 65(4), 237.

Schweitzer, R., Melville, F., Steel, Z., \& Lacherez, P. (2006). Trauma, post-migration living difficulties and social support as predictors of psychological adjustment in resettled Sudanese refugee. Australian and New Zealand Journal of Psychiatry, 40(2), 179-187.

Shrout, P. E., \& Bolger, N. (2002). Mediation in experimental and nonexperimental studies: new procedures and recommendations. Psychological Methods, 7(4), $422-445$.

Silove, D., Sinnerbrink, I., Field, A. Manicavasagar, V., \& Steel, Z. (1997). Anxiety, depression and PTSD in asylum-seekers: Associations with pre-migration trauma and post-migration stressors. British Journal of Psychiatry, 170, 351-357.

Silove, D. (1999). The psychosocial effects of torture, mass human rights violations and refugee trauma: toward an integrated conceptual framework. The Journal of Nervous and Mental Disease. 189, 200-207.

Silove, D., Steel, Z., Suslsik, I., Frommer, N., Loneragan, C., Brooks, R., le Touze, D., Manicavasagar, V., Ceollo, M., Smith, M., \& Harris, E. (2006). Torture, mental 
health status and the outcomes of refugee applications among recently arrived asylum seekers in Australia. International Journal of Migration, Health and Social Care, 2(1), 4-14.

Simpkins, S. D., \& Riggs, N. R. (2014). Cultural competence in afterschool programs. New Directions for Youth Development, 2014(144), 105-117. doi:10.1002/yd.20116

Sinan, A., \& Alkan, B. B. (2015). A useful approach to identify the multicollinearity in the presence of outliers. Journal of Applied Statistics, 42(5), 986-993.

Smedley, B. D., Stith, A. Y., \& Nelson, A. R. (2002). Unequal treatment: Confronting racial and ethnic health disparities in health care. Washington, DC: Institute of Medicine, National Academy Press.

Smith, D. K. (n.d). Cross-cultural adjustment. University of California Education Abroad Program. Retrieved from http://eap.ucop.edu/Documents/_forms/CrossCultural_Adjustment_Article.pdf

Smith, L. S. (1998). Concept analysis: Cultural competence. Journal of Cultural Diversity, 5, 4-10.

Sriramesh, K., Grunig, J., \& Buffington, J. (1992). Corporate culture and public relations. In J. E. Grunig (Ed.), Excellence in public relations and communication management (pp. 577-596). Hillsdale, NJ: Lawrence Erlbaum Associates, Inc.

Stage, F. K., Carter, H. C., \& Nora, A. (2004). Path analysis: An introduction and analysis of a decade of research. The Journal of Educational Research, 98(1), 513. 
Starbuck, W. H. (2003). The origins of organization theory. In H. Tsoukas \& C. Knudsen (Eds.), The oxford handbook of organization theory (1st ed., pp. 143-182). New York: Oxford University Press. doi:10.1093/oxfordhb/9780199275250.0

Starr, S., \& Wallace, D. C. (2009). Self-reported cultural competence of public health nurses in a Southeastern U.S. public health department. Public Health Nursing, 26(1), 48-57.

Steel, Z., Momartin, S., Silove, D., Coello, M., Aroche, J., \& Tay K. Y. (2011). Two year psychosocial and mental health outcomes for refugees subjected to restrictive or supportive immigration policies. Social Science \& Medicine, 72, 1149-1156.

Stiffman, A. R., Hadley-Ives, E., Dore, P., Polgar, M., Horvath, V. E., Striley, C., et al. (2000). Youths' access to mental health services: The role of providers' training, resource connectivity, and assessment of need. Mental Health Service Research, 2, 141-154.

Stiffman, A. R., Striley, C. W., Horvath, V. E., Hadely-Ives, E., Polgar, M., Elze, D., \& Pescarino, R. (2001). Organizational context and provider perception as determinants of mental health service use. The Journal of Behavioral Health Services \& Research, 28(2), 188-204.

Stiffman, A. R., Pescosolido, B., \& Cabassa, L. J. (2004). Building a model to understand youth service access: The Gateway Provider Model. Mental Health Service Research, 6(4), 189-198.

Stiffman, A. R., Freedenthal, S., Dore, P., Ostmann, E., Osborne, V., \& Silmere, H. (2006). The role of providers in mental health services offered to AmericanIndian youths. Psychiatric Services, 57(8), 1185-1191. 
Suarez-Balcazar, Y., Baicazar, F., Taylor-Ritzier, T., Portillo, N., Rodakowsk, J., GarciaRamirez, M., \& Willis, C. (2011). Development and validation of the cultural competence assessment instrument: A factorial analysis. Journal of Rehabilitation, 77(1), 4-13.

Suh, E. E. (2004). The model of cultural competence through an evolutionary concept analysis. Journal of Transcultural Nursing, 15, 93-102.

Sundvall, A., Tidemalm, D. H., Titelman, D. E., Runeson, B., \& Bäärnhielm, S. (2015). Assessment and treatment of asylum seekers after a suicide attempt: A comparative study of people registered at mental health services in a Swedish location. BMC Psychiatry, 15, 235.

Suphanchaimat, R., Kantamaturapoj, K., Putthasri, W., \& Prakongsai, P. (2015). Challenges in the provision of healthcare services for migrants: A systematic review through providers' lens. BMC Health Services Research, 15, 390. doi:10.1186/s12913-015-1065-z

Suurmond, J., Seeleman, C., Rupp, I., Goosen, S., \& Stronks, K. (2010). Cultural competence among nurse practitioners working with asylum seekers. Nurse Education Today, 30(8), 821-826. doi:10.1016/j.nedt.2010.03.006

Syracuse.com. (2017, January 27). Syracuse refugee program will be 'effectively shut down' by Trump order. Retrieved from http://www.syracuse.com/politics/index.ssf/2017/01/syracuse refugee program will be effectively shut down by trump_order.html

Tabachnick, B. G. (2013). Using multivariate statistics (6th ed.). New York, NY: Pearson Education. 
Tastsoglou, E., Abidi, C. B., Brigham, S. M., \& Lange, E. A. (2014). (en) gendering vulnerability: Immigrant service providers' perceptions of needs, policies, and practices related to gender and women refugee claimants in Atlantic Canada. Refuge, 30(2), 67-78.

Taylor, R. (2005). Addressing barriers to cultural competence. Journal for Nurses in Staff Development, 21(4), 135-144.

Taylor, F. W. (1913). The principle of scientific management. New York: Harper \& Brother.

Terheggen, M. A., Stroebe, M. S., \& Kleber, R. J. (2001). Western conceptualization and Eastern experience: A cross-cultural study of traumatic stress reactions among Tibetan refugee in India. Journal of Traumatic Stress, 14(2), 391-403.

The Boston Blobe. (2017, March 7). Chart: The percentage of women and men in each profession. Retrieved from https://www.bostonglobe.com/metro/2017/03/06/chart-the-percentage-womenand-men-each-profession/GBX22YsW10XaeHghwXfE4H/story.html

The New York Times. (2017, January 27). How Trump's Executive Order Will Affect the U.S. Refugee Program. Retrieved from https://www.nytimes.com/interactive/2017/01/25/us/politics/trump-refugeeplan.html

The Washington Post. (2017, February 15). Citing Trump, evangelical agency that resettles refugees to lay off 140 staffers, close five locations. Retrieved from https://www.washingtonpost.com/news/acts-of-faith/wp/2017/02/15/citing-trump- 
evangelical-agency-that-resettles-refugees-to-lay-off-140-staff-close-fivelocations/?utm_term $=.74098759$ fdad

The White House (2015, November 20). Infographic: The Screening Process for Refugee Entry into the United States. Retrieved from https://www.whitehouse.gov/blog/2015/11/20/infographic-screening-processrefugee-entry-united-states

Thompson, C. G., Kim, R. S., Aloe, A. M., \& Becker, B. J. (2017). Extracting the variance inflation factors and other multicollinearity diagnostics from typical regression results. Basic and Applied Social Psychology, 39(2), 81-90.

Tran, T. V. (1992). Adjustment among different age and ethnic group of Indochinese in the United States. The Gerontologist, 32(4), 508-518.

Truong, M., Gibbs, L., Paradies, Y., \& Priest, N. (2017). “Just treat everybody with respect": Health service providers' perspectives on the role of cultural competence in community health service provision. ABNF Journal, 28(2), 34-43. United Nation High Commissioner for Refugee (UNHCR). (2011). Refugees. Retrieved from http://www.unhcr.org/pages/49c3646c125.html

United Nation High Commissioner for Refugee (UNHCR). (2014). The United States of America: By the government of the United States of America; Country chaptersUNHCR Resettlement handbook. Retrieved from http://www.unhcr.org/3c5e5a764.html

United Nation High Commissioner for Refugee (UNHCR). (2017). Figures at a glance. Retrieved from http://www.unhcr.org/en-us/figures-at-a-glance.html. 
United Nation High Commissioner for Refugee (UNHCR) (n.d.). Resettlement: New beginnings in a new country. Retrieved from http://www.unhcrcentraleurope.org/en/what-we-do/resettlement.html

United States Census Bureau, Geography Division. (n.d.). Census regions and divisions of the United States. Retrieved from http://www2.census.gov/geo/pdfs/mapsdata/maps/reference/us regdiv.pdf

U.S. Citizenship and Immigration Services. (2016). The United States refugee admissions program (USRAP) consultation \& worldwide processing priorities. Retrieved from https://www.uscis.gov/humanitarian/refugees-asylum/refugees/united-statesrefugee-admissions-program-usrap-consultation-worldwide-processing-priorities

Uribe Guajardo, M. G., Slewa-Younan, S., Kitchener, B. A., Mannan, H., Mohammad, Y., \& Jorm, A. F. (2018). Improving the capacity of community-based workers in Australia to provide initial assistance to Iraqi refugees with mental health problems: An uncontrolled evaluation of a Mental Health Literacy Course. International Journal of Mental Health System, 12(2).

U. S. Citizenship and Immigration Service (USCIS). (n.d.). Refugee \& Asylum. Retrieved from https://www.uscis.gov/humanitarian/refugees-asylum U. S. Citizenship and Immigration Service (USCIS). (n.d.). Act 101(a)15P. Retrieved from \slb $\backslash$ SERVICE LAW BOOKS MENU \IMMIGRATION AND NATIONALITY ACT \INA: ACT 101 - DEFINITIONS \Act 101(a)15P

U.S. Department of Health and Human Services, Office of Minority Health. (2002). National standards for culturally and linguistically appropriate service in health 
care: Final Report. Retrieved from

http://minorityhealth.hhs.gov/assets/pdf/checked/finalreport.pdf

U.S. Department of State (2013). U.S. Refugee admissions program FAQs. Retrieved from http://www.state.gov/j/prm/releases/factsheets/2013/210135.htm

van de Luitgaarden, G. M. J. (2009). Evidence-based practice in social work: Lessons from judgment and decision-making theory. British Journal of Social Work, 39(2), 243-260. doi:10.1093/bjsw/bcm117

Van Keer, R. L., Deschepper, R., Francke, A. L., Huyghens, L., \& Bilsen, J. (2015). Conflicts between healthcare professionals and families of multi-ethnic patient population during critical care: An ethnographic study. Critical Care 19, 441-454.

Verbeke, W., Volgering, M., \& Hessels, M. (1998). Exploring the conceptual expansion within the field of organizational behaviour: Organizational climate and organizational culture. Journal of Management Studies, 35(3), 303-329. doi:10.1111/1467-6486.00095

Ward, C., Bochner, S., \& Furnham, A. (2001). The psychology of culture shock $\left(2^{\text {nd }}\right.$ ed.). Hove, UK: Routledge.

Watters, C. (2001). Emerging paradigms in the mental health care of refugees. Social Science and Medicine, 52, 1709-1718.

Weine, S. M., Kuc, G., Dzudza, E., Razzano, L., \& Pavkovic, I. (2001). PTSD among Bosnian refugees: A survey of providers' knowledge, attitudes and service patterns. Community Mental Health Journal, 37(3), 261-271. doi:10.1023/A:1017533214935 
Wilbur, V. (2008). Factors that influence the cultural competence of nurse practitioner students (Doctoral Dissertation). Widener University.

Williams, R. (2015). Measurement error 2: Scale construction (Very brief overview). University of Notre Dame. Retrieved from https://www3.nd.edu/ rwilliam/stats2/123.pdf

Wong-Tam, M. (2003). National Settlement Service and Standards Framework. Retrieved from http://www.orgwise.ca/sites/osi.ocasi.org.stage/files/resources/National\%20Settle ment $\% 20$ Service\%20and\%20Standards\%20Framework.pdf

Yaun, K., \& Zhong, X. (2013). Robustness of fit indices and leverage observations in structural equation modeling. Psychological Methods, 18(2), 121-136.

Yazdi-Feyzabadi, V., Emami, M., \& Mehrolhassani, M. H. (2015). Health information system in primary health care: The challenges and barriers from local providers' perspective of an area in Iran. International Journal of Preventive Medicine, 6 , $57-66$.

Yi, J., Kim, M. A., Kim, K., \& Hong, J. S. (2016). Exploring social service providers' perspectives on barriers to social services for early adjustment of immigrant adolescents in South Korea. Journal of Immigrant and Minority Health, 18(5), 1076-1084.

Yoon, E., Lee, R. M., \& Goh, M. (2008). Acculturation, social connectedness, and subjective well-being. Cultural Diversity and Ethnic Minority Psychology, 14(3), $246-255$. 


\section{APPENDIX A: LIST OF ORGANIZATIONS}

\section{Northeast (16)}

- Connecticut - Catholic charities

- Connecticut - Integrated Refugee \& Immigrant Services

- Connecticut - International Institute

- Maine - Catholic charities

- Massachusetts - Catholic charities (Boston)

- New Jersey - Catholic charities (Camden)

- New York - Interfaith Works

- New York - Journey's End Refugee Services

- New York - International Institute

- New York - International Rescue Committee

- New York - U. S. Committee for Refugees and Immigrants (USCRI)

- Pennsylvania - Catholic charities (Pittsburgh, Scranton, and Harrisburg)

- Pennsylvania - HIAS

- Pennsylvania - Church World Services (CWS)

- Pennsylvania - U. S. Committee for Refugees and Immigrants (USCRI)

- Vermont - U. S. Committee for Refugees and Immigrants (USCRI)

\section{Midwest (35)}

- Illinois - Refugee One

- Illinois - Lutheran Social Services

- Illinois - World Relief

- Indiana - Lutheran Social Services

- Indiana - Catholic charities

- Indiana - The Life Ahead

- Iowa - Catholic charities

- Iowa - U. S. Committee for Refugees and Immigrants (USCRI)

- Kansas - Catholic charities

- Kansas - Jewish Vocational Service

- Kansas - Della Lamb Community Service

- Kansas - International Rescue Committee

- Kansas - The Episcopal Dioceses (Wichita)

- Michigan - Catholic charities

- Michigan - Samaritas

- Michigan - Bethany Christian Services

- Michigan - St. Vincent Catholic Charities

- Michigan - U. S. Committee for Refugees and Immigrants (USCRI)

- Minnesota - Catholic charities

- Minnesota - International Institute

- Minnesota - Lutheran Social Services

- Missouri - Catholic charities

- Missouri - International Institute 
- Nebraska - Lutheran Family Services

- Ohio - Catholic charities (Miami Valley, Southwestern Ohio, and Cleveland)

- Ohio - US Together

- Ohio - Community Refugee and Immigration Services

- Ohio - International Institute

- Ohio - World Relief

- Oklahoma - Catholic charities (Oklahoma city)

- St. Louis - International Institute

- Wisconsin - Catholic charities (Milwaukee and Green Bay)

- Wisconsin - International Institute

- Wisconsin - Lutheran Social Services

- Wisconsin - World Relief

\section{South (34)}

- Alabama - Catholic charities (Birmingham)

- Arkansas - Catholic charities (Springdale)

- Florida - Catholic charities

- Florida - Lutheran Social Services

- Florida - International Rescue Committee

- Florida - Jewish Family Services

- Georgia - Catholic charities (Atlanta)

- Georgia - Lutheran Social Services

- Georgia - International Rescue Committee

- Kentucky - Catholic charities (Louisville)

- Kentucky - Refugee Ministries, INC.

- Louisiana - Catholic charities (Lafayette, Baton Rouge, and New Orleans)

- Maryland - World Relief

- Maryland - International Rescue Committee

- Mississippi - Catholic charities (Jackson)

- North Carolina - Catholic charities (Charlotte)

- North Carolina - Carolina Refugee Resettlement Agency

- North Carolina - Lutheran Family Services

- North Carolina - U. S. Committee for Refugees and Immigrants (USCRI)

- North Carolina - World Relief

- North Carolina - Church World Services (CWS)

- Tennessee - Catholic charities (Nashville)

- Tennessee - Bridge Refugee Services

- Tennessee - World Relief

- Texas - Catholic charities (San Antonio, Houston, and Dallas)

- Texas - Refugee Services

- Texas - Caritas

- Texas - Alliance for Multicultural Community Services

- Texas - Interfaith Ministries

- Texas - International Rescue Committee

- Virginia - Lutheran Social Services 
- Virginia - International Rescue Committee

- Virginia - Church World Services (CWS)

- Virginia - Catholic charities (Arlington and Richmond)

\section{West (24)}

- Arizona - Phoenix-Refugee Focus

- Arizona - International Rescue Committee

- Arizona - Lutheran Social Services

- Arizona (Southern) - Catholic Community Service

- California - Catholic charities

- California - World Relief

- California - Jewish Family Services

- California - International Rescue Committee

- Colorado Springs / Denver - Lutheran Family Services

- Idaho - Refugee Center

- Idaho - International Rescue Committee

- Nevada - Catholic charities

- New Mexico - Catholic charities

- Oregon - Catholic charities

- Oregon - Lutheran Community Service

- Utah - Catholic charities

- Utah - International Rescue Committee

- Washington - World Relief

- Washington - Jewish Family Services

- Washington - International Rescue Committee

- Washington - Catholic charities

- Washington - Diocese of Olympia

- Washington - Lutheran Community Services

- Washington - U. S. Committee for Refugees and Immigrants (USCRI) 


\section{APPENDIX B: REFUGEE ARRIVALS BY STATE}

Refugee arrivals from October 1, 2015 through August 31, 2016

1. Northeast

\begin{tabular}{|c|c|c|}
\hline Region & State & Number of Refugee \\
\hline \multirow{9}{*}{ Northeast } & Connecticut & 737 \\
\hline & Maine & 487 \\
\hline & Massachusetts & 1,453 \\
\hline & New Hampshire & 414 \\
\hline & New Jersey & 453 \\
\hline & New York & 4,285 \\
\hline & Pennsylvania & 2,733 \\
\hline & Vermont & 325 \\
\hline & Total & 10,887 \\
\hline
\end{tabular}

2. Midwest

\begin{tabular}{l|l|c}
\multicolumn{1}{|c|}{ State } & Number of Refugee \\
\hline \multirow{4}{*}{ Region } & \multicolumn{1}{|c}{ Illinois } & 2,607 \\
\cline { 2 - 3 } & Indiana & 1,652 \\
\cline { 2 - 3 } & Iowa & 901 \\
\cline { 2 - 3 } & Kansas & 766 \\
\cline { 2 - 3 } & Michigan & 3,551 \\
\cline { 2 - 3 } & Minnesota & 2,358 \\
\cline { 2 - 3 } & Missouri & 1,648 \\
\cline { 2 - 3 } & Nebraska & 1,156 \\
\cline { 2 - 3 } & North Dakota & 500 \\
\cline { 2 - 3 } & Ohio & 3,614 \\
\cline { 2 - 3 } & South Dakota & 360 \\
\cline { 2 - 3 } & Wisconsin & 1,424 \\
\cline { 2 - 3 } & \multicolumn{1}{c}{ Total } & 20,537
\end{tabular}

3. South

\begin{tabular}{l|l|c}
\multicolumn{1}{|c|}{ State } & Number of Refugee \\
\hline \multirow{5}{*}{ South } & Alabama & 100 \\
\cline { 2 - 3 } & Arkansas & 1 \\
\cline { 2 - 3 } & Florida & 2,495 \\
\cline { 2 - 3 } & Georgia & 2,596 \\
\cline { 2 - 3 } & Kentucky & 2,089 \\
\hline
\end{tabular}




\begin{tabular}{l|l|c}
\hline \multirow{2}{*}{} & Louisiana & 162 \\
\cline { 2 - 3 } & Maryland & 1,428 \\
\cline { 2 - 3 } & Mississippi & 9 \\
\cline { 2 - 3 } & North Carolina & 2,745 \\
\cline { 2 - 3 } & Oklahoma & 496 \\
\cline { 2 - 3 } & South Carolina & 308 \\
\cline { 2 - 3 } & Tennessee & 1,591 \\
\cline { 2 - 3 } & Texas & 6,736 \\
\cline { 2 - 3 } & Virginia & 1,313 \\
\cline { 2 - 3 } & West Virginia & 25 \\
\cline { 2 - 2 } \multicolumn{2}{|c}{ Total } & 22,094
\end{tabular}

4. West

\begin{tabular}{|c|c|c|}
\hline Region & State & Number of Refugee \\
\hline \multirow{11}{*}{ West } & Arizona & 3,726 \\
\hline & California & 6,463 \\
\hline & Colorado & 1,426 \\
\hline & Idaho & 980 \\
\hline & Montana & 6 \\
\hline & Nevada & 608 \\
\hline & New Mexico & 245 \\
\hline & Oregon & 1,110 \\
\hline & Utah & 1,026 \\
\hline & Washington & 2,818 \\
\hline & Total & 18,408 \\
\hline
\end{tabular}




\section{APPENDIX C: RECRUITMENT MAIL}

** If you have not employment, education, health care, or resettlement related job title and have not provided any services to refugees over the past 12 months, please ignore this email. Thank you.

You have been selected to participate in a study about refugee service providers. This study is being conducted by Eunyoung Jang, a graduate student from the University of Missouri, School of Social Work. I want to identify the key factors that are important for service providers when providing services for refugees. The purpose of this study is to contribute towards achieving a greater understanding of refugee service providers' experiences and how the services for refugees can be improved. If you decide to participate, then you will be asked to complete some questionnaires relating to your information, cultural competence level, and your agency's environment. The questionnaires should take about 15 - 20 minutes to complete. You will not be asked to identify yourself on any of the forms. You are free to refuse to answer any questions that make you feel uncomfortable. After the completing the survey, you will receive a gift certificate for $\$ 5.00$ to thank you for participating in this project.

Your participation in this study is voluntary, and the results will be confidential and will not be shared with anyone other than the research team. Your name will not appear on any of the information that is reported. If you are interested in assisting me in this study, please start your survey by following the attached link. Moreover, if you know anybody who may be eligible to participate in this survey, please feel free to share this link. 
Finally, if you have any questions concerning your rights as a research participant, please contact Eunyoung Jang (ej3mf@mail.missouri.edu) and Mansoo Yu, dissertation chair (yuma@missouri.edu).

Thank you for your assistance. Your participation is very much appreciated. 


\section{APPENDIX D: SURVEY FORM}

Dear Participant:

My name is Eunyoung Jang and I am a doctoral student in the Department of Social Work at the University of Missouri-Columbia. I am conducting a study to explore what factors contribute to refugee service provider when they provide services. I would like to invite you to join this research study.

What is this study about?

The purpose of this study is to understand the important factors and characteristic that influence to service provision for refugees.

\section{What is being asked of you?}

If you decide to take part in this project, continue on this website to complete the survey. They survey should take 15-20 minutes to complete and asks for some information about your knowledge of refugee and community resources, cultural competence level and some background information about you and your organization.

\section{What are the benefits of the survey?}

I hope you find value in reflecting on service provision in your organization. In addition, the results of the study may be of interest to you in that it may provide direction for policy makers and organizations to improve the refugee service provision to better meet the needs of refugees. Upon request, I will send a copy of results. A $\$ 5$ gift card will be provided to thank you for your time.

\section{Are there risks to me of I am in the study?}

Some individuals could experience minor psychological discomfort while completing the survey. Your participation is voluntary and you are free to withdraw at any time. If you experience any discomfort while completing the survey. Great care will be taken to minimize these risks by the researcher. Please feel free to contact Eunyoung Jang to discuss your concerns.

\section{While my information be kept private?}

The information in this survey is being collected anonymously. No one will be able to connect your responses to you. The result of this study may be published or presented at professional meeting, but the identities of all research participants will remain anonymous.

\section{Who can I talk to if I have questions?}

If you have questions about this study or the information in this form, please contact Eunyoung Jang (ej3mf@mail.missouri.edu) or Dr. Mansoo Yu, dissertation chair (yuma@missouri.edu).

Thank you again, to assist me in my educational endeavors. Your insight and information are very valuable to us. The result from this study may be helpful to people who help others solve their problems in the community in helping you and others here in United States. 
What does my clicking "accept" on this form mean?

You understand the information given to you in this form.

You are able to ask the researcher questions and state any concerns

The researcher will respond to your questions and concerns

You believe you understand the research study and the potential benefits and risks that are involved

\section{Accept}

[ ] 


\section{Questionnaire}

Screening Question

1.Do you provide service to Refugee?

[ ] Yes $->$ if Yes, please Continue the survey

[ ] No -> If No, please DO NOT Continue. Thank you for your concerning

2. Does your job title include any of the following categories?

[ Employment, Education, Health care, or Resettlement ]

[ ] Yes $\rightarrow$ if Yes, please Continue the survey on the next page

[ ] No -> If No, please DO NOT Continue. Thank you for your concerning

\section{Section 1: Demographic Information}

The following questions focus on demographic information about you and your organization. Please answer the questions as completely as you can.

1. When were you born? Years

2. What is your biological sex?
[ ] Female
[ ] Male
[ ] Other

3. Please specify your race/ethnicity.
[ ] White
[ ] Native American or American Indian
[ ] Hispanic or Latino
[ ] Asian / Pacific Islander
[ ] Black or African American
[ ] Other 
4. I was a refugee
[ ] Yes
[ ] No

5. What is your highest qualification/education level?
[ ] Less than high school
[ ] Graduate degree
[ ] High school (GED)
[ ] Other (Please specify)
[ ] Undergraduate degree

6. What state does your organization serve?

7. What type of agency do you represent?

(e.g. Refugee and Immigration Services, Social Service Agency, Healthcare agency, etc.)

8. Approximately, How much percentage of Refugee clients are in total number of your agency's client population? $\%$

9. Approximately, How many groups have you served in the past 12 months?

10. How many years have you worked at this agency?

11. What is your job title?

12. Approximately, How many employees work at your agency? 


\section{Section 2: Services to Refugees}

13. Please put indicated number how many times you have been provided to refugees or referred to other agencies the following services in the past 12 months. (23 items)

[Service Provided: the number of times direct service is provided for refugees]

[Service Referrals: the number of times indirect service is provided for refugees]

0: None

1: 1 -10 times

2: $11-20$ times

3: 21-30 times

4: 31-40 times

5: $41-50$ times

6: More than 51 times

\begin{tabular}{|l|l|l|}
\hline \multicolumn{1}{|c|}{ Service } & Service provided & Service referrals \\
\hline Housing & & \\
\hline Food & & \\
\hline Furnishing & & \\
\hline Household supplies & & \\
\hline Clothing & & \\
\hline Airport reception & & \\
\hline Transportation & & \\
\hline Translation services & & \\
\hline Case management & & \\
\hline Job training & & \\
\hline Employment services & & \\
\hline Financial support & & \\
\hline Social security & & \\
\hline Food stamp & & \\
\hline ESL class enrollment & & \\
\hline School enrollment & & \\
\hline Medical support (e.g., doctor's appointment) & & \\
\hline Health insurance & & \\
\hline Health screening & & \\
\hline
\end{tabular}




\begin{tabular}{|l|l|l|}
\hline Cultural orientation & & \\
\hline Health safety orientation & & \\
\hline Immigration legal services & & \\
\hline Religious services & & \\
\hline
\end{tabular}

\section{Section 3: Personal factors}

13. The following questions in this section focus on your awareness regarding refugees. In your opinion, are you agree or disagree with following statement?? (7) items)

\begin{tabular}{|l|l|l|}
\hline \multicolumn{1}{|c|}{ Items } & Agree & Disagree \\
\hline $\begin{array}{l}\text { Refugees are generally people outside of their country who are } \\
\text { unable or unwilling to return home because of they fear serious } \\
\text { harm }\end{array}$ & & \\
\hline $\begin{array}{l}\text { Refugee, asylum seeker, and immigrant are the same meaning } \\
\text { in a broad sense }\end{array}$ & & \\
\hline $\begin{array}{l}\text { Less than 1\% of global refugee population applicants who are } \\
\text { strong candidates for resettlement }\end{array}$ & & \\
\hline $\begin{array}{l}\text { In 2015, the three largest refugee population in the United } \\
\text { States were Burma, Iraq, and Somalia }\end{array}$ & & \\
\hline $\begin{array}{l}\text { In 2015, around 70,000 refugees were officially resettled into } \\
\text { the United States }\end{array}$ & & \\
\hline $\begin{array}{l}\text { In response to safety issue, the Obama administration } \\
\text { proposed to significantly decrease the number of refugees the } \\
\text { United States accepts following years. }\end{array}$ & & \\
\hline $\begin{array}{l}\text { Refugee can apply for lawful permanent resident status (also as } \\
\text { known as a green card) as soon as being admitted to the United } \\
\text { States }\end{array}$ & & \\
\hline
\end{tabular}

14. The following questions in this section focus on your awareness regarding your community resources. How familiar are you with following statement? (20 items)

\begin{tabular}{|c|c|c|c|c|}
\hline Items & $\begin{array}{c}\text { Not } \\
\text { familiar } \\
\text { at all }\end{array}$ & $\begin{array}{c}\text { Barely } \\
\text { familiar }\end{array}$ & $\begin{array}{c}\text { Fairly } \\
\text { familiar }\end{array}$ & $\begin{array}{c}\text { Very } \\
\text { familiar }\end{array}$ \\
\hline How familiar are you with: & 1 & 2 & 3 & 4 \\
\hline TANF program &
\end{tabular}




\begin{tabular}{|c|c|c|c|c|}
\hline Food stamp program & 1 & 2 & 3 & 4 \\
\hline Medicaid program & 1 & 2 & 3 & 4 \\
\hline Food bank & 1 & 2 & 3 & 4 \\
\hline One-time cash assistance & 1 & 2 & 3 & 4 \\
\hline Schools & 1 & 2 & 3 & 4 \\
\hline ESL program & 1 & 2 & 3 & 4 \\
\hline Job training & 1 & 2 & 3 & 4 \\
\hline $\begin{array}{l}\text { Other education program } \\
\text { (e.g. Parenting, sports, and } \\
\text { music) }\end{array}$ & 1 & 2 & 3 & 4 \\
\hline Hospital & 1 & 2 & 3 & 4 \\
\hline Community clinic & 1 & 2 & 3 & 4 \\
\hline Public health organization & 1 & 2 & 3 & 4 \\
\hline Drug or alcohol clinic & 1 & 2 & 3 & 4 \\
\hline Mental health clinic & 1 & 2 & 3 & 4 \\
\hline Church & 1 & 2 & 3 & 4 \\
\hline Ethnic organization & 1 & 2 & 3 & 4 \\
\hline Social worker & 1 & 2 & 3 & 4 \\
\hline Counselor & 1 & 2 & 3 & 4 \\
\hline Psychologist & 1 & 2 & 3 & 4 \\
\hline Lawyer & 1 & 2 & 3 & 4 \\
\hline
\end{tabular}

15. The following questions in this section focus on your cultural competence. Please check your best response to each item. There are no right or wrong answers. (28 items)

\begin{tabular}{|l|c|c|c|c|}
\hline \multicolumn{1}{|c|}{ Items } & Never & $\begin{array}{c}\text { At } \\
\text { times }\end{array}$ & Often & Always \\
\hline $\begin{array}{l}\text { I include cultural assessment when I do } \\
\text { refugee evaluations }\end{array}$ & 1 & 2 & 3 & 4 \\
\hline
\end{tabular}




\begin{tabular}{|c|c|c|c|c|}
\hline $\begin{array}{l}\text { I seek information on cultural needs when I } \\
\text { identify new refugees in my practice }\end{array}$ & 1 & 2 & 3 & 4 \\
\hline $\begin{array}{l}\text { I have resource books and other materials } \\
\text { available to help me learn about refugees from } \\
\text { different cultures }\end{array}$ & 1 & 2 & 3 & 4 \\
\hline $\begin{array}{l}\text { I use a variety of sources to learn about the } \\
\text { cultural heritage of other people }\end{array}$ & 1 & 2 & 3 & 4 \\
\hline $\begin{array}{l}\text { I ask refugees to tell me about their own } \\
\text { explanations of problems }\end{array}$ & 1 & 2 & 3 & 4 \\
\hline $\begin{array}{l}\text { I ask refugees to tell me about their } \\
\text { expectations for service }\end{array}$ & 1 & 2 & 3 & 4 \\
\hline $\begin{array}{l}\text { I avoid using generalizations to stereotype } \\
\text { groups of people }\end{array}$ & 1 & 2 & 3 & 4 \\
\hline $\begin{array}{l}\text { I recognize potential barriers to service that } \\
\text { might be encountered by different people }\end{array}$ & 1 & 2 & 3 & 4 \\
\hline $\begin{array}{l}\text { I act to remove obstacles for people of } \\
\text { different cultures when I identify such } \\
\text { obstacles }\end{array}$ & 1 & 2 & 3 & 4 \\
\hline $\begin{array}{l}\text { I act to remove obstacles for people of } \\
\text { different cultures when refugees identify such } \\
\text { obstacles to me }\end{array}$ & 1 & 2 & 3 & 4 \\
\hline $\begin{array}{l}\text { I welcome feedback from refugees about how I } \\
\text { relate to others with different cultures }\end{array}$ & 1 & 2 & 3 & 4 \\
\hline $\begin{array}{l}\text { I welcome feedback from co-workers about } \\
\text { how I relate to others with different cultures }\end{array}$ & 1 & 2 & 3 & 4 \\
\hline $\begin{array}{l}\text { I find ways to adapt my services to refugees } \\
\text { cultural preferences }\end{array}$ & 1 & 2 & 3 & 4 \\
\hline I document cultural assessment & 1 & 2 & 3 & 4 \\
\hline $\begin{array}{l}\text { I document the adaptations I make with } \\
\text { refugees }\end{array}$ & 1 & 2 & 3 & 4 \\
\hline $\begin{array}{l}\text { I learn from my co-workers about people with } \\
\text { different cultural heritage }\end{array}$ & 1 & 2 & 3 & 4 \\
\hline Items & $\begin{array}{l}\text { Strongly } \\
\text { Disagree }\end{array}$ & Disagree & Agree & $\begin{array}{c}\text { Strongly } \\
\text { Agree }\end{array}$ \\
\hline $\begin{array}{l}\text { Race is the most important factor in } \\
\text { determining a person's culture - } \mathrm{R}\end{array}$ & 1 & 2 & 3 & 4 \\
\hline
\end{tabular}




\begin{tabular}{|l|c|c|c|c|}
\hline $\begin{array}{l}\text { People with a common cultural background } \\
\text { think and act alike }\end{array}$ & 1 & 2 & 3 & 4 \\
\hline Many aspects of culture influence services & 1 & 2 & 3 & 4 \\
\hline $\begin{array}{l}\text { Aspects of cultural diversity need to be } \\
\text { assessed for each individual, group, and } \\
\text { organization }\end{array}$ & 1 & 2 & 3 & 4 \\
\hline $\begin{array}{l}\text { If I know about a person's culture, I do not } \\
\text { need to assess their personal preferences for } \\
\text { services -R }\end{array}$ & 1 & 2 & 3 & 4 \\
\hline $\begin{array}{l}\text { Spirituality and religious beliefs are important } \\
\text { aspects of many cultural groups }\end{array}$ & 1 & 2 & 3 & 4 \\
\hline $\begin{array}{l}\text { Individuals may identify with more than one } \\
\text { cultural group }\end{array}$ & 1 & 2 & 3 & 4 \\
\hline $\begin{array}{l}\text { Language barriers are the only difficulties for } \\
\text { recent refugees to the United States -R }\end{array}$ & 1 & 2 & 3 & 4 \\
\hline $\begin{array}{l}\text { I understand that people from different } \\
\text { cultures may define the concept of “services" } \\
\text { in different ways }\end{array}$ & 1 & 2 & 3 & 4 \\
\hline $\begin{array}{l}\text { I think that knowing about different cultural } \\
\text { groups helps direct my work with individuals, } \\
\text { families, groups, and organizations }\end{array}$ & 1 & 2 & 3 & 4 \\
\hline $\begin{array}{l}\text { I enjoy working with people who are culturally } \\
\text { different from me }\end{array}$ & 1 & 2 & 3 & 4 \\
\hline
\end{tabular}

\section{Section 4: Organizational Culture Factor (42 items)}

16. The following questions in this section focus on your organizations' culture. Please check your best response to each item. There are no right or wrong answers.

\begin{tabular}{|l|c|c|c|c|}
\hline \multicolumn{1}{|c|}{ Item } & $\begin{array}{c}\text { Not } \\
\text { at all }\end{array}$ & $\begin{array}{c}\text { A slight } \\
\text { extent }\end{array}$ & $\begin{array}{c}\text { A great } \\
\text { extent }\end{array}$ & $\begin{array}{c}\text { A very } \\
\text { great } \\
\text { extent }\end{array}$ \\
\hline $\begin{array}{l}\text { Members of my organizational unit are } \\
\text { expected to improve the well-being of each } \\
\text { client }\end{array}$ & 1 & 2 & 3 & 4 \\
\hline $\begin{array}{l}\text { Members of my organizational unit are } \\
\text { expected to evaluate how much we benefit } \\
\text { clients }\end{array}$ & 1 & 2 & 3 & 4 \\
\hline
\end{tabular}




\begin{tabular}{|c|c|c|c|c|}
\hline $\begin{array}{l}\text { Members of my organizational unit are } \\
\text { expected to place the well-being of clients' } \\
\text { first }\end{array}$ & 1 & 2 & 3 & 4 \\
\hline $\begin{array}{l}\text { Members of my organizational unit are } \\
\text { expected to find ways to serve clients more } \\
\text { effectively }\end{array}$ & 1 & 2 & 3 & 4 \\
\hline $\begin{array}{l}\text { Members of my organizational unit are } \\
\text { expected to act in the best interest of each } \\
\text { client }\end{array}$ & 1 & 2 & 3 & 4 \\
\hline $\begin{array}{l}\text { Members of my organizational unit are } \\
\text { expected to be available to each client we } \\
\text { serve }\end{array}$ & 1 & 2 & 3 & 4 \\
\hline $\begin{array}{l}\text { Members of my organizational unit are } \\
\text { expected to be responsive to the needs of } \\
\text { each client }\end{array}$ & 1 & 2 & 3 & 4 \\
\hline $\begin{array}{l}\text { Members of my organizational unit are } \\
\text { expected to have up-to-date knowledge }\end{array}$ & 1 & 2 & 3 & 4 \\
\hline $\begin{array}{l}\text { Members of my organizational unit are } \\
\text { expected to become more effective in } \\
\text { serving clients }\end{array}$ & 1 & 2 & 3 & 4 \\
\hline $\begin{array}{l}\text { Members of my organizational unit are } \\
\text { expected to learn new tasks }\end{array}$ & 1 & 2 & 3 & 4 \\
\hline $\begin{array}{l}\text { Members of my organizational unit are } \\
\text { expected to interact positively with others }\end{array}$ & 1 & 2 & 3 & 4 \\
\hline $\begin{array}{l}\text { Members of my organizational unit are } \\
\text { expected to strive for excellence }\end{array}$ & 1 & 2 & 3 & 4 \\
\hline $\begin{array}{l}\text { Members of my organizational unit are } \\
\text { expected to be number one }\end{array}$ & 1 & 2 & 3 & 4 \\
\hline $\begin{array}{l}\text { Members of my organizational unit are } \\
\text { expected to plan for success }\end{array}$ & 1 & 2 & 3 & 4 \\
\hline $\begin{array}{l}\text { Members of my organizational unit are } \\
\text { expected to pay attention to detail }\end{array}$ & 1 & 2 & 3 & 4 \\
\hline $\begin{array}{l}\text { I have to ask a supervisor or coordinator } \\
\text { before I do almost anything }\end{array}$ & 1 & 2 & 3 & 4 \\
\hline $\begin{array}{l}\text { A person can make his or her own decisions } \\
\text { without checking with anyone else- reverse } \\
\text { coded }\end{array}$ & 1 & 2 & 3 & 4 \\
\hline $\begin{array}{l}\text { Any decision I make has to have a } \\
\text { supervisor's or coordinator's approval }\end{array}$ & 1 & 2 & 3 & 4 \\
\hline $\begin{array}{l}\text { There is only one way to do the job-the } \\
\text { boss's way }\end{array}$ & 1 & 2 & 3 & 4 \\
\hline
\end{tabular}




\begin{tabular}{|c|c|c|c|c|}
\hline $\begin{array}{l}\text { Whenever we have a problem, we are } \\
\text { supposed to go to the same person for an } \\
\text { answer }\end{array}$ & 1 & 2 & 3 & 4 \\
\hline $\begin{array}{l}\text { People here always get their orders from } \\
\text { higher up }\end{array}$ & 1 & 2 & 3 & 4 \\
\hline $\begin{array}{l}\text { There can be little action until a supervisor or } \\
\text { coordinator approves the decision }\end{array}$ & 1 & 2 & 3 & 4 \\
\hline $\begin{array}{l}\text { The same steps must be followed in } \\
\text { processing every piece of work }\end{array}$ & 1 & 2 & 3 & 4 \\
\hline $\begin{array}{l}\text { We usually work under the same } \\
\text { circumstances day to day }\end{array}$ & 1 & 2 & 3 & 4 \\
\hline $\begin{array}{l}\text { People here do the same job on the same } \\
\text { way everyday }\end{array}$ & 1 & 2 & 3 & 4 \\
\hline $\begin{array}{l}\text { How things are done around here is left } \\
\text { pretty much up to the person doing the work } \\
\text { - } \mathrm{R}\end{array}$ & 1 & 2 & 3 & 4 \\
\hline $\begin{array}{l}\text { The same procedures are to be followed in } \\
\text { most situations }\end{array}$ & 1 & 2 & 3 & 4 \\
\hline $\begin{array}{l}\text { I feel that I am my own boss in most matter - } \\
\mathrm{R}\end{array}$ & 1 & 2 & 3 & 4 \\
\hline $\begin{array}{l}\text { We are to follow strict operating procedures } \\
\text { at all times }\end{array}$ & 1 & 2 & 3 & 4 \\
\hline $\begin{array}{l}\text { Members of my organizational unit are } \\
\text { expected to not make waves }\end{array}$ & 1 & 2 & 3 & 4 \\
\hline $\begin{array}{l}\text { Members of my organizational unit are } \\
\text { expected to stay uninvolved }\end{array}$ & 1 & 2 & 3 & 4 \\
\hline $\begin{array}{l}\text { Members of my organizational unit are } \\
\text { expected to be stern and unyielding }-\mathrm{R}\end{array}$ & 1 & 2 & 3 & 4 \\
\hline $\begin{array}{l}\text { Members of my organizational unit are } \\
\text { expected to avoid problems }\end{array}$ & 1 & 2 & 3 & 4 \\
\hline $\begin{array}{l}\text { Members of my organizational unit are } \\
\text { expected to follow rather than lead }\end{array}$ & 1 & 2 & 3 & 4 \\
\hline $\begin{array}{l}\text { Members of my organization unit are } \\
\text { expected to be thoughtful and considerate - } \\
\text { reverse coded }\end{array}$ & 1 & 2 & 3 & 4 \\
\hline $\begin{array}{l}\text { Members of my organizational unit are } \\
\text { expected to be critical }\end{array}$ & 1 & 2 & 3 & 4 \\
\hline $\begin{array}{l}\text { Members of my organizational unit are } \\
\text { expected to avoid being different }\end{array}$ & 1 & 2 & 3 & 4 \\
\hline $\begin{array}{l}\text { Members of my organizational unit are } \\
\text { expected to be dominant and assertive }-\mathrm{R}\end{array}$ & 1 & 2 & 3 & 4 \\
\hline
\end{tabular}




\begin{tabular}{|l|c|c|c|c|}
\hline $\begin{array}{l}\text { Members of my organizational unit are } \\
\text { expected to criticize mistakes }\end{array}$ & 1 & 2 & 3 & 4 \\
\hline $\begin{array}{l}\text { Members of my organizational unit are } \\
\text { expected to defeat the competition }\end{array}$ & 1 & 2 & 3 & 4 \\
\hline $\begin{array}{l}\text { Members of my organizational unit are } \\
\text { expected to be competitive with coworkers }\end{array}$ & 1 & 2 & 3 & 4 \\
\hline $\begin{array}{l}\text { Members of my organizational unit are } \\
\text { expected to go along with group decisions }\end{array}$ & 1 & 2 & 3 & 4 \\
\hline
\end{tabular}

Thank you for completing this Survey.

\section{APPENDIX E: RESIDUAL PLOT}

Service provided residual Histogram

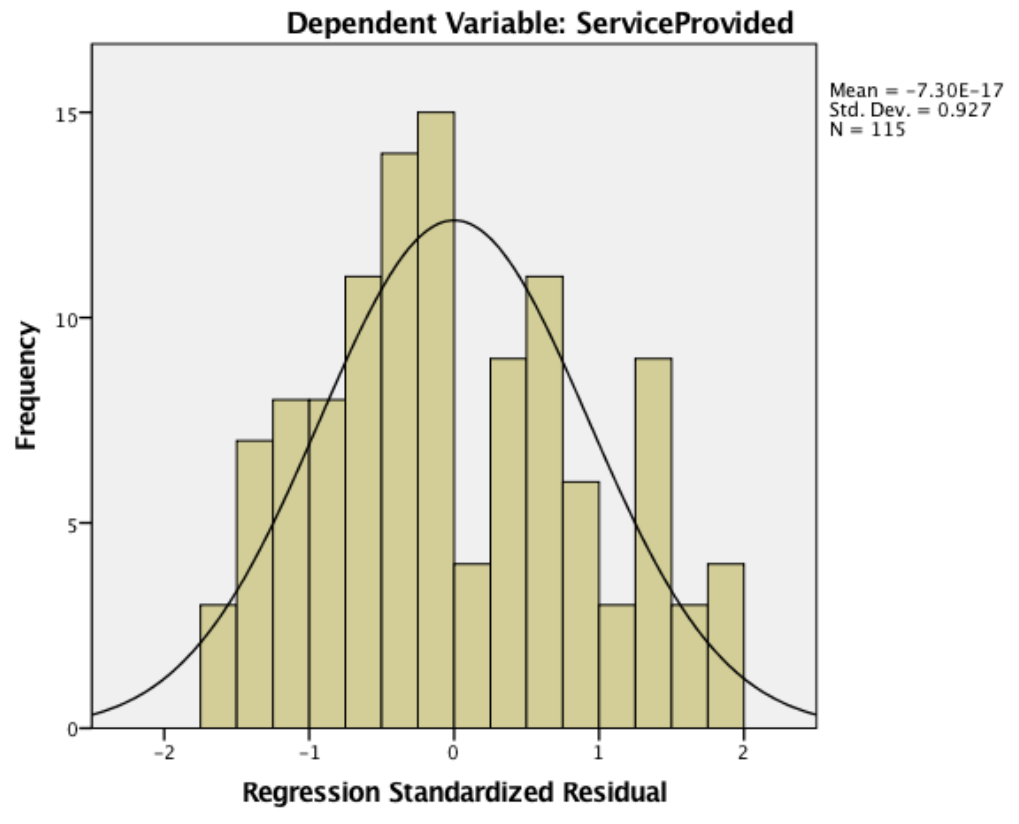

Service provided residual P-P Plot 


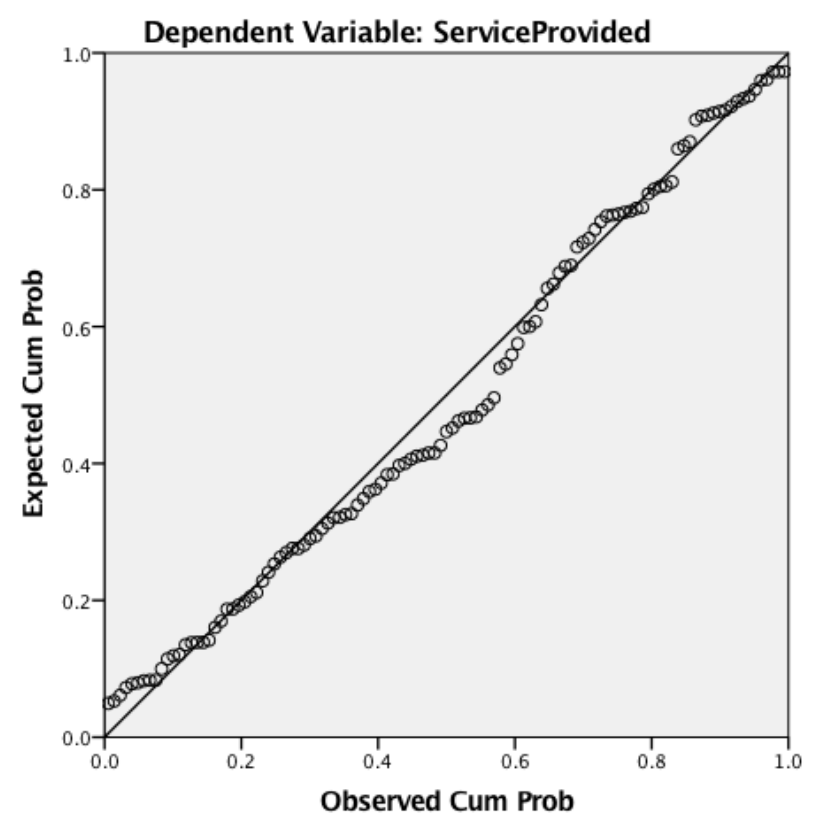

Service referral residual Histogram

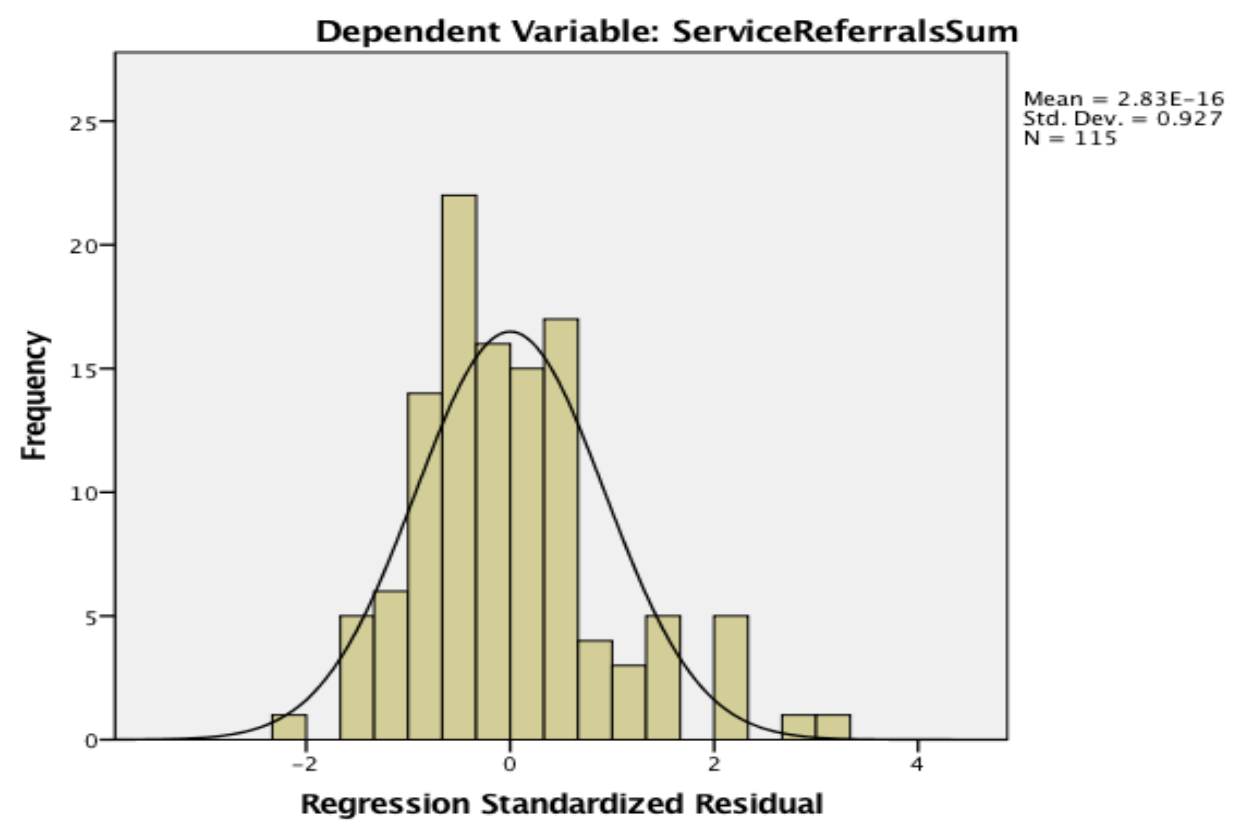

Service referral residual P-P Plot 


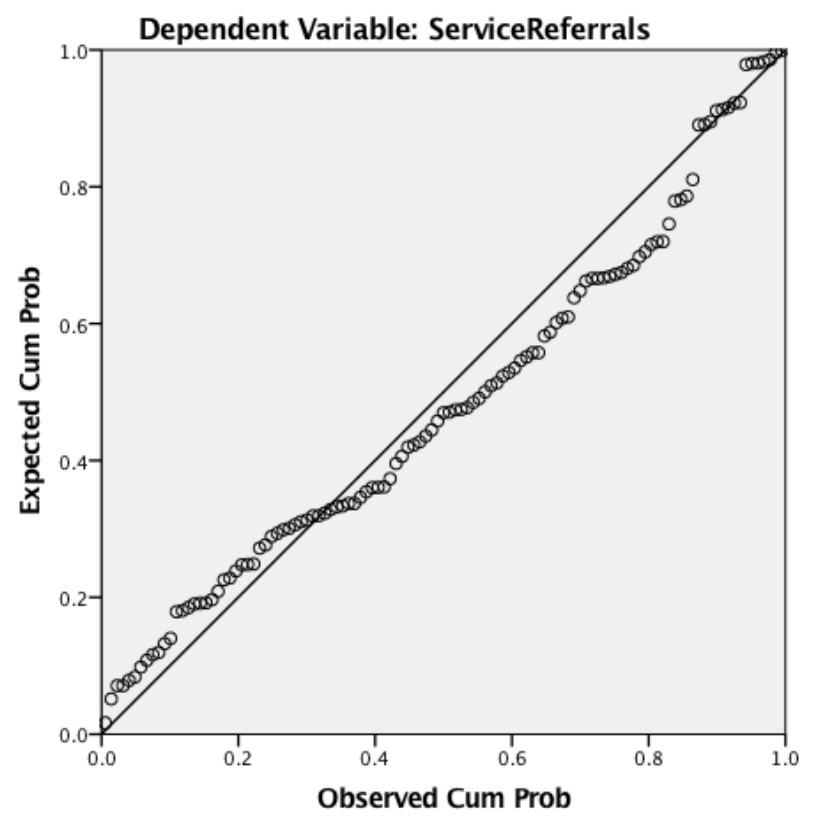

\section{VITA}

Eunyoung Jang was born in Busan, South Korea. She joined Seoul Women’s University, where she received a Bachelor's degree in Gerontology in 2005 and a Master's degree in Social Work (MSW) in 2010. While studying in the MSW program, she worked as a research assistant on a project to develop cultural diversity measurement. Moreover, she completed two internships at an assisted living facility and social service center. Through this project and the internships, Eunyoung realized that she has a passion to help people from diverse backgrounds and knew more about social work study to advocate for the underserved population. Therefore, she decided to move to the United States to learn more about the social work through diverse culture and people. In 2010, she was admitted for the second MSW at the University of Missouri and she successfully completed her two practicums with the social service center and refugee and immigration 
services. The experience that she gained during the two practicums helped her expand her perspective and gain an insight of her research interest. Eunyoung then pursued a Ph.D. in Social Work at University of Missouri, Columbia, to strengthen her skills in research and teaching; and there she worked as research and teaching assistant. Her next step is to become an Assistant Professor at the University of Wisconsin's Whitewater School of Social Work, beginning in August 2018. Her research interests include cultural competence, social support, health, access and utilization of services, and promotion of well-being and social justice for refugees and the immigrant populations. 